\title{
Lifting harmonic morphisms I: metrized complexes and Berkovich skeleta
}

\author{
Omid Amini ${ }^{1}$, Matthew Baker ${ }^{2 *}$, Erwan Brugallé ${ }^{3}$ and Joseph Rabinoff ${ }^{2}$
}

\author{
*Correspondence: \\ mbaker@math.gatech.edu \\ ${ }^{2}$ School of Mathematics, Georgia \\ Institute of Technology, Atlanta GA \\ 30332-0160, USA \\ Full list of author information is \\ available at the end of the article
}

\begin{abstract}
Let $K$ be an algebraically closed, complete non-Archimedean field. The purpose of this paper is to carefully study the extent to which finite morphisms of algebraic $K$-curves are controlled by certain combinatorial objects, called skeleta. A skeleton is a metric graph embedded in the Berkovich analytification of $X$. A skeleton has the natural structure of a metrized complex of curves. We prove that a finite morphism of $K$-curves gives rise to a finite harmonic morphism of a suitable choice of skeleta. We use this to give analytic proofs of stronger 'skeletonized' versions of some foundational results of Liu-Lorenzini, Coleman, and Liu on simultaneous semistable reduction of curves. We then consider the inverse problem of lifting finite harmonic morphisms of metrized complexes to morphisms of curves over $K$. We prove that every tamely ramified finite harmonic morphism of $\Lambda$-metrized complexes of $k$-curves lifts to a finite morphism of $K$-curves. If in addition the ramification points are marked, we obtain a complete classification of all such lifts along with their automorphisms. This generalizes and provides new analytic proofs of earlier results of Saïdi and Wewers. As an application, we discuss the relationship between harmonic morphisms of metric graphs and induced maps between component groups of Néron models, providing a negative answer to a question of Ribet motivated by number theory.

This article is the first in a series of two. The second article contains several applications of our lifting results to questions about lifting morphisms of tropical curves.
\end{abstract}

\section{Introduction}

This article is the first in a series of two. The second, entitled Lifting harmonic morphisms II: tropical curves and metrized complexes, will be cited as [3]; references of the form "Theorem II.1.1" will refer to Theorem 1.1 in [3].

Throughout this paper, unless explicitly stated otherwise, $K$ denotes a complete algebraically closed non-Archimedean field with nontrivial valuation val : $K \rightarrow \mathbf{R} \cup\{\infty\}$. Its valuation ring is denoted $R$, its maximal ideal is $\mathfrak{m}_{R}$, and the residue field is $k=R / \mathfrak{m}_{R}$. We denote the value group of $K$ by $\Lambda=\operatorname{val}\left(K^{\times}\right) \subset \mathbf{R}$.

The purpose of this paper is to carefully study the extent to which finite morphisms of algebraic $K$-curves are controlled by certain combinatorial objects, called skeleta. Let $X$ be a smooth, proper, connected $K$-curve. Roughly speaking, a triangulation $(X, V \cup D)$ of $X$ (with respect to a finite set of punctures $D \subset X(K)$ ) is a finite set $V$ of points in the Berkovich analytification $X^{\text {an }}$ of $X$ whose removal partitions $X^{\text {an }}$ into open balls

(c) 2015 Amini et al.; licensee Springer. This is an Open Access article distributed under the terms of the Creative Commons

Attribution License (http://creativecommons.org/licenses/by/4.0), which permits unrestricted use, distribution, and reproduction in any medium, provided the original work is properly credited. 
and finitely many open annuli (with the punctures belonging to distinct open balls). Triangulations of $(X, D)$ are naturally in one-to-one correspondence with semistable models $\mathfrak{X}$ of $(X, D)$ : see Section 5. A triangulation $(X, V \cup D)$ gives rise to a skeleton $\Sigma(X, V \cup D)$ of $X$. The skeleton of a triangulated punctured curve is the dual graph of the special fiber $\mathfrak{X}_{k}$ of the corresponding semistable model, equipped with a canonical metric, along with completed infinite rays in the directions of the punctures. There are many skeleta in $X^{\text {an }}$, although if $X \backslash D$ is hyperbolic (i.e., has negative Euler characteristic $\chi(X, D)=2-2 g(X)-\# D)$, then there exists a unique minimal skeleton. A skeleton of $(X, D)$ is by definition a subset $\Sigma \subset X^{\text {an }}$ of the form $\Sigma(X, V \cup D)$ for some triangulation of $X$.

\subsection{Skeletal simultaneous semistable reduction}

First we will prove that finite morphisms of curves induce morphisms of skeleta:

Theorem 1.2. Let $\varphi: X^{\prime} \rightarrow X$ be a finite morphism of smooth, proper, connected $K$-curves and let $D \subset X(K)$ be a finite set. There exists a skeleton $\Sigma \subset X$ such that $\Sigma^{\prime}:=\varphi^{-1}(\Sigma)$ is a skeleton of $X^{\prime}$. For any such $\Sigma$, the map $\varphi: \Sigma^{\prime} \rightarrow \Sigma$ is a finite harmonic morphism of metric graphs.

See Corollaries 4.18 and 4.28. Harmonic morphisms of graphs are defined in Section 2. Due to the close relationship between semistable models and skeleta, Theorem 1.2 should be interpreted as a very strong simultaneous semistable reduction theorem. In fact, we will show how to formally derive from Theorem 1.2 the simultaneous semistable reduction theorems of Liu-Lorenzini [32], Coleman [23], and Liu [34]. Our version of these results hold over any non-Archimedean field $K_{0}$, not assumed to be discretely valued. As an example, the following weak form of Liu's theorem is a consequence of Theorem 1.2:

Corollary 1. Let $X, X^{\prime}$ be smooth, proper, geometrically connected curves over a nonArchimedean field $K_{0}$, and let $\varphi: X^{\prime} \rightarrow X$ be a finite morphism. Then, there exists a finite, separable extension $K_{1}$ of $K_{0}$ and semistable models $\mathfrak{X}, \mathfrak{X}^{\prime}$ of the curves $X_{K_{1}}, X_{K_{1}}^{\prime}$, respectively, such that $\varphi_{K_{1}}$ extends to a finite morphism $\mathfrak{X}^{\prime} \rightarrow \mathfrak{X}$.

We discuss simultaneous semistable reduction theorems in Section 5. We wish to emphasize that Theorem 1.2 does not follow from any classical simultaneous semistable reduction theorem, in that there exist finite morphisms $\varphi: \mathfrak{X}^{\prime} \rightarrow \mathfrak{X}$ of semistable models such that the inverse image of the corresponding skeleton of $X$ is not a skeleton of $X^{\prime}$. (See, however, [24] where a skeletal version of Liu's theorem is derived from Liu's method of proof in the discretely valued case.) Our proof of Theorem 1.2 is entirely analytic, resting on an analysis of morphisms between open annuli and open balls; in particular, it makes almost no reference to semistable reduction theory and is therefore quite different from Liu and Liu-Lorenzini's approach.

A skeletal simultaneous semistable reduction theorem is important precisely when one wants to obtain a well-behaved morphism of graphs from a finite morphism of curves. This is crucial for obtaining the spectral lower bound on the gonality in [24], for instance. 


\subsection{Lifting harmonic morphisms}

Our second goal is in a sense inverse to the first: we wish to lift finite harmonic morphisms of metric graphs to finite morphisms of curves. More precisely, let $(X, D)$ be a punctured $K$-curve as above, let $\Sigma$ be a skeleton, and let $\bar{\varphi}: \Sigma^{\prime} \rightarrow \Sigma$ be a finite harmonic morphism of metric graphs. It is natural to ask whether there exists a curve $X^{\prime}$ and a finite morphism $\varphi: X^{\prime} \rightarrow X$ such that $\varphi^{-1}(\Sigma)$ is a skeleton of $X^{\prime}$ and $\varphi^{-1}(\Sigma) \cong \Sigma^{\prime}$ as metric graphs over $\Sigma$. In general, the answer is 'no': there are subtle 'Hurwitz obstructions' to such a lift, as described below. One solution is to enrich $\Sigma, \Sigma^{\prime}$ with the extra structure of metrized complexes of curves. A metrized complex of curves is basically a metric graph $\Gamma$ and for every (finite) vertex $p \in \Gamma$, the data of a smooth, proper $k$-curve $C_{p}$, along with an identification of the edges adjacent to $p$ with distinct $k$-points of $C_{p}$. There is a notion of a finite harmonic morphism of metrized complexes of curves, which consists of a finite harmonic morphism $\varphi: \Gamma^{\prime} \rightarrow \Gamma$ of underlying metric graphs, and for every vertex $p^{\prime} \in \Gamma^{\prime}$ with $p=\varphi\left(p^{\prime}\right)$, a finite morphism $\varphi_{p^{\prime}}: C_{p^{\prime}} \rightarrow C_{p}$, satisfying various compatibility conditions.

Now, let $\Sigma=\Sigma(X, V \cup D)$ for a triangulated punctured curve $(X, V \cup D)$. We will show that $\Sigma$ is naturally a metrized complex of curves, and that finite harmonic morphisms $\Sigma^{\prime} \rightarrow \Sigma$ of metrized complexes do lift to finite morphisms of curves, under a mild tameness hypothesis. Moreover, we have very precise control over the set of such lifts entirely in terms of the morphism of metrized complexes. The main lifting theorem, stated somewhat imprecisely, is as follows:

Theorem 1.3 (Lifting theorem). Let $(X, V \cup D)$ be a triangulated punctured $K$-curve, let $\Sigma$ be its skeleton, and let $\Sigma^{\prime} \rightarrow \Sigma$ be a tame covering of metrized complexes of curves. Then, there exists a curve $X^{\prime}$ and a finite morphism $\varphi: X^{\prime} \rightarrow X$, branched only over $D$, such that $\varphi^{-1}(\Sigma)$ is a skeleton of $\left(X^{\prime}, \varphi^{-1}(D)\right)$ and $\varphi^{-1}(\Sigma) \cong \Sigma^{\prime}$ as metrized complexes of curves over $\Sigma$. There are finitely many such lifts $X^{\prime}$ up to $X$-isomorphism. There are explicit descriptions of the set of lifts and the automorphism group of each lift (as a cover of $X$ ) in terms of the morphism $\Sigma^{\prime} \rightarrow \Sigma$.

See Theorems 7.4, 7.7, and 7.10 for the precise statements. Tame coverings are defined in Definition 2.21. The hardest part of the proof of Theorem 1.3 is a local lifting result, Theorem 6.18, in which the target curve $X$ is replaced by a neighborhood of a vertex $p \in \Sigma$. Theorem 6.18 eventually reduces to classical results about the tamely ramified étale fundamental group.

It is worth mentioning that given a metrized complex of curves $\mathcal{C}$ with edge lengths contained in $\Lambda$, it is not hard to construct a triangulated punctured $K$-curve $(X, V \cup D)$ such that $\Sigma(X, V \cup D) \cong \mathcal{C}$. See Theorem 3.24.

Our lifting results extend several theorems in the literature, as well as making them more precise. Saïdi ([39], Théoréme 3.7) proves a version of the lifting theorem for semistable formal curves (without punctures) over a complete discrete valuation ring. His methods also make use of the tamely ramified étale fundamental group and analytic gluing arguments. Wewers [47] works more generally with marked curves over a complete Noetherian local ring using deformation-theoretic arguments, proving that every tamely ramified admissible cover of a marked semistable curve over the residue field of a complete Noetherian local ring lifts. Wewers classifies the possible lifts (fixing, as we 
do, a lift of the target) in terms of certain non-canonical 'deformation data' depending on compatible choices of formal coordinates at the nodes on both the source and target curves.

Our results have several advantages compared to these previous approaches. For one, we are able to work over non-Noetherian rank-1 valuation rings and descend the lifting theorems a posteriori to an essentially arbitrary non-Archimedean field: see (7.11). The real novelty, however, is the systematic use of metrized complexes of curves in the formulation of the lifting theorems, in particular the classification of the set of lifts $X^{\prime}$ of $\Sigma^{\prime} \rightarrow \Sigma$. For example, in our situation, Wewers' non-canonical deformation data are replaced by certain canonical gluing data, which means that the automorphism group of the morphism of metrized complexes acts naturally on the set of gluing data (but not on the set of deformation data). This is what allows us to classify lifts up to isomorphism as covers of the target curve, as well as determine the automorphism group of such a lift. Saïdi's lifting theorem is more similar to ours, with generators of inertia groups over nodal points playing the role of gluing data. It is not clear to the authors whether his methods can be extended to give a classification of lifts.

The question of lifting (and classification of all possible liftings) in the wildly ramified case is more subtle and cannot be guaranteed in general. See Remark 7.6.

\subsection{Homomorphisms of component groups}

We will discuss in Section 8 the relationship between harmonic morphisms of metric graphs and component groups of Néron models, connecting our lifting results to natural questions in arithmetic algebraic geometry. The idea is as follows. Let $X$ be a smooth, proper, geometrically connected curve over a discretely valued field $K_{0}$, and suppose that $X$ admits a semistable model $\mathfrak{X}$ over the valuation ring $R_{0}$ of $K_{0}$. Let $J$ be the Jacobian of $X$, and let $\Phi_{X}$ be the group of connected components of the special fiber of the Néron model of $J$.

Given a metric graph $\Gamma$ with integer edge lengths, there is a naturally associated abelian group Jac $\operatorname{~reg}(\Gamma)$, called its regularized Jacobian. The association $\Gamma \mapsto \operatorname{Jac}_{\mathrm{reg}}(\Gamma)$ satisfies Picard and Albanese funtoriality: that is, given a finite harmonic morphism $\Gamma^{\prime} \rightarrow \Gamma$, there are natural push-forward and pullback homomorphisms Jac $\operatorname{reg}_{\text {reg }}\left(\Gamma^{\prime}\right) \rightarrow \operatorname{Jac}_{\mathrm{reg}}(\Gamma)$ and $\operatorname{Jac}_{\text {reg }}(\Gamma) \rightarrow \operatorname{Jac}_{\text {reg }}\left(\Gamma^{\prime}\right)$.

A series of theorems of Raynaud, translated into our language, imply that $\Phi_{X} \cong$ $\operatorname{Jac}_{\text {reg }}\left(\Sigma_{\mathfrak{X}}\right)$ canonically, where $\Sigma_{\mathfrak{X}}$ is the skeleton induced by the semistable model $\mathfrak{X}$. We observe that this isomorphism respects both Picard and Albanese functoriality.

For modular curves such as $X_{0}(N)$ with $N$ prime, the minimal regular model has a special fiber consisting of two projective lines intersecting transversely. Maps from such curves to elliptic curves, and their induced maps on component groups of Néron models, play an important role in arithmetic geometry; for example, Ribet's work establishing that the Shimura-Taniyama conjecture implies Fermat's Last Theorem was motivated by the failure of such induced maps on component groups to be surjective in general. (The cokernel of such maps controls congruences between modular forms.) Ribet noticed that if one considers instead the case where the target curve has genus at least 2 , then in all examples he knew of, the induced map on component groups was surjective, and in personal communication with the second author, he asked whether this was a general property of curves whose reduction looks like that of $X_{0}(N)$. As a concrete 
application of Theorem 1.3, the next proposition provides a negative answer to this question.

Proposition 1.5. There exists a finite morphism $f: X^{\prime} \rightarrow X$ of semistable curves over a discretely valued field $K_{0}$ with $g(X) \geq 2$ such that:

- the special fiber of the minimal regular model of $X^{\prime}$ consists of two projective lines intersecting transversely;

- the induced map $f_{*}: \Phi_{X^{\prime}} \rightarrow \Phi_{X}$ on component groups of Néron models of Jacobians is not surjective.

In order to prove Proposition 1.5, we proceed as follows. As component groups can be calculated in terms of metric graphs, one first finds a finite harmonic morphism of metric graphs $\varphi: \Gamma^{\prime} \rightarrow \Gamma$ such that $\Gamma^{\prime}$ has two vertices and at least three edges, all of which have length one, and such that $\varphi_{*}: \operatorname{Jac}_{\mathrm{reg}}\left(\Gamma^{\prime}\right) \rightarrow \operatorname{Jac}_{\text {reg }}(\Gamma)$ is not surjective. One then enriches $\varphi$ to a morphism of metrized complexes of curves, with rational residue curves, and uses Theorem 3.24 and Theorem 1.3 (along with a descent argument) to lift this to a finite morphism of curves $X^{\prime} \rightarrow X$. Such a morphism satisfies the conditions of Proposition 1.5.

\subsection{Applications to tropical lifting theorems}

Tropical geometry has many applications to algebraic geometry, in particular enumerative algebraic geometry. The basic strategy is to associate a combinatorial 'tropical' object to an algebraic object, and prove that counting the former is somehow equivalent to counting the latter. An important ingredient in any such argument is a tropical lifting theorem, which is a precise description of the algebraic lifts of a given tropical object.

Many of the intended applications of our lifting results are contained in the second paper [3], in which we prove a number of tropical lifting theorems. The skeleton of a curve, viewed as a metric graph, is a tropical object associated to the curve; our lifting results can therefore be interpreted as tropical lifting theorems for morphisms of metric graphs, in the following sense. Recall that $\Lambda \subset \mathbf{R}$ is the value group of $K$. A $\Lambda$-metric graph is a metric graph $\Gamma$ whose edge lengths are contained in $\Lambda$. We say that a finite harmonic morphism $\bar{\varphi}: \Gamma^{\prime} \rightarrow \Gamma$ of $\Lambda$-metric graphs is liftable provided that there exists a finite morphism of $K$-curves $\varphi: X^{\prime} \rightarrow X$, a set of punctures $D \subset X(K)$, and a skeleton $\Sigma$ of $(X, D)$ such that $\Sigma \cong \Gamma$ as metric graphs, $\varphi^{-1}(\Sigma)$ is a skeleton of $\left(X^{\prime}, \varphi^{-1}(D)\right)$, and $\Sigma^{\prime} \cong \Gamma^{\prime}$ as metric graphs over $\Sigma \cong \Gamma$. Theorem 1.3 (along with Theorem 3.24) shows that the obstruction to lifting a morphism of metric graphs $\bar{\varphi}: \Gamma^{\prime} \rightarrow \Gamma$ to a morphism of curves is the same as the obstruction to enriching $\varphi$ with the structure of a morphism of metrized complexes of curves. The following more precise statement is an immediate consequence of Proposition 7.15 and Theorem 3.24:

Corollary 1.6. Assume char $(k)=0$. Let $\bar{\varphi}: \Gamma^{\prime} \rightarrow \Gamma$ be a finite harmonic morphism of $\Lambda$-metric graphs. Suppose that $\varphi$ can be enriched to a morphism of metrized complexes of curves. Then, $\varphi$ is liftable.

We remark that we actually take advantage of the stronger form of Theorem 1.3, in particular the calculation of the automorphism group of a lift, when lifting group actions on metrized complexes in [3]. 
The problem of enriching a morphism of metric graphs to a morphism of metrized complexes is essentially equivalent to the problem of assigning $k$-curves $C_{p}, C_{p^{\prime}}$ to vertices $p \in \Gamma, p^{\prime} \in \Gamma^{\prime}$ and finding morphisms of $k$-curves $C_{p^{\prime}} \rightarrow C_{p}$ with prescribed ramification profiles. The latter is a question about whether certain Hurwitz numbers are nonzero. See Proposition II.3.3. Allowing arbitrary choices of residue curves $C_{p}, C_{p^{\prime}}$, this can always be done:

Theorem. Suppose that char $(k)=0$. Any finite harmonic morphism $\varphi: \Gamma^{\prime} \rightarrow \Gamma$ of $\Lambda$-metric graphs is liftable to a morphism of curves.

See Theorem II.3.11. Imposing requirements on the lifted curves $X, X^{\prime}$ give rise to variants of this tropical lifting problem. The most basic such requirement is to specify the genera of the curves. An augmented metric graph is a metric graph $\Gamma$ along with an integer $g(p) \in \mathbf{Z}_{\geq 0}$ for every finite vertex $p$ of $\Gamma$, called the genus. The genus of an augmented graph $\Gamma$ is defined to be

$$
g(\Gamma)=h_{1}(\Gamma)+\sum_{p} g(p)
$$

where $h_{1}(\Gamma)$ is the first Betti number. Any metrized complex of curves has an underlying augmented metric graph, where $g(p):=g\left(C_{p}\right)$, the genus of the residue curve. If $\Sigma$ is a skeleton of a curve $X$, viewed as an augmented metric graph, then we have $g(\Sigma)=g(X)$.

A finite harmonic morphism of augmented metric graphs $\Gamma^{\prime} \rightarrow \Gamma$ is liftable if the morphism of underlying metric graphs is liftable to a finite morphism of curves $X^{\prime} \rightarrow X$ such that the isomorphism of $\Gamma$ (resp. $\Gamma^{\prime}$ ) with a skeleton of $X$ (resp. $\left.X^{\prime}\right)$ respects the augmented metric graph structure. In this case, $g(X)=g(\Gamma)$ and $g\left(X^{\prime}\right)=g\left(\Gamma^{\prime}\right)$. By our lifting theorem, $\Gamma^{\prime} \rightarrow \Gamma$ is liftable to a morphism of curves if and only if it can be enhanced to a morphism of metrized complexes such that $g\left(C_{p}\right)=g(p)$ for every finite vertex. Now the obstruction to lifting is nontrivial: there exist finite harmonic morphisms of metric graphs which cannot be enhanced to a morphism of metrized complexes. This corresponds to the emptiness of certain Hurwitz spaces over $k$. See Section II.5 for the following striking example of this phenomenon:

Theorem. There exists an augmented metric graph $\Gamma$ admitting a finite morphism of degree $d>0$ to an augmented metric graph $T$ of genus zero but which is not isomorphic to a skeleton of a d-gonal curve $X$.

In [3], we also give applications of the lifting theorem to the following kinds of tropical lifting problems, among others:

- When $T$ is an augmented metric graph of genus zero, we study a variant of the lifting problem in which the genus of the source curve is prescribed, but the degree of the morphism is not. We use this to prove that linear equivalence of divisors on a tropical curve $C$ coincides with the equivalence relation generated by declaring that the fibers of every finite harmonic morphism from $C$ to the tropical projective line are equivalent.

- We study liftability of metrized complexes equipped with a finite group action, and as an application classify all (augmented) metric graphs arising as the skeleton of a 
hyperelliptic curve. As mentioned above, this study actually takes advantage of the more precise form of Theorem 1.3, not just the existence of a lift.

\subsection{Organization of the paper}

In Section 2, we recall some basic definitions of graphs with additional structures, including metrized complexes of curves, and define harmonic morphisms between them. We provide somewhat more detail than is strictly necessary for the exposition in this paper but will prove necessary in [3].

In Section 3, we review the structure theory of analytic curves and define the skeleton of a curve. We show that the skeleton is naturally a metrized complex of curves, and we prove that any metrized complex arises in this way. In Section 4, we develop 'relative' versions of the results of Section 3. We prove that a finite morphism of curves induces a finite morphism between suitable choices of skeleta, and that the map on skeleta is a finite harmonic morphism of metrized complexes of curves. In Section 5, we show how the previous two sections can be used to derive various simultaneous semistable reduction theorems.

In Section 6, we prove a local lifting theorem, which essentially says that a tame covering of metrized complexes of curves can be lifted in a unique way to a tame covering of analytic $K$-curves in a neighborhood of a vertex. This is the technical heart of the lifting theorem. In Section 7, we globalize the considerations of the previous section, giving the classification of lifts of a tame covering of metrized complexes of curves to a tame covering of triangulated punctured $K$-curves.

Section 8 contains an example application, in which we construct a morphism of curves over a discretely valued field such that the covariantly associated morphism of component groups of Jacobians has a prescribed behavior. We use this to answer a question of Ribet.

\subsection{Related work}

Another framework for the foundations of tropical geometry has been proposed by Kontsevich-Soibelman [30,31] and Mikhalkin [35,36], in which tropical objects are associated to real one-parameter families of complex varieties. We refer to [30,31] for some conjectures relating this framework to Berkovich spaces. In this setting, the notion of metrized complex of curves is similar to the notion of phase-tropical curves, and Proposition 7.15 is a consequence of Riemann's Existence Theorem. We refer the interested reader to the forthcoming paper (Mikhalkin: Phase-tropical curves I. Realizability and enumeration, forthcoming) for more details (see also [17] where this statement is implicit, as well as Corollary II.3.8).

Harmonic morphisms of finite graphs were introduced by Urakawa in [45] and further explored in [8]. Harmonic morphisms of metric graphs have been introduced independently by several different people. Except for the integrality condition on the slopes, they appear already in Anand's paper [4]. The definition we use is the same as the one given in $[21,35]$.

Finite harmonic morphisms of metrized complexes can be regarded as a metrized version of the notion of admissible cover due to Harris and Mumford [29] (where, in addition, arbitrary ramification above smooth points is allowed). Recall that for two semistable curves $Y^{\prime}$ and $Y$ over a field $k$, a finite surjective morphism $\varphi: Y^{\prime} \rightarrow Y$ is an admissible cover if $\varphi^{-1}\left(Y^{\text {sing }}\right)=Y^{\prime \text { sing }}$ and for each singular point $y^{\prime}$ of $Y^{\prime}$, the ramification indices 
at $y^{\prime}$ along the two branches intersecting at $y^{\prime}$ coincide (In addition, one usually imposes that all the other ramifications of $\varphi$ are simple). An admissible cover naturally gives rise to a finite harmonic morphism of metrized complexes: denoting by $\mathcal{C}$ the regularization of $Y$ (the metrized complex associated to $Y$ in which each edge has length one), define $\mathcal{C}^{\prime}$ as the metrized complex obtained from $Y^{\prime}$ by letting the length of the edge associated to the double point $y^{\prime}$ be $1 / r_{y^{\prime}}$ (where $r_{y^{\prime}}$ is the ramification index of $\varphi$ at $y^{\prime}$ along either of the two branches). The morphism $\varphi$ of semistable curves naturally extends to a finite harmonic morphism $\varphi: \mathcal{C}^{\prime} \rightarrow \mathcal{C}$ : on each edge $e^{\prime}$ of $\mathcal{C}^{\prime}$ corresponding to a double point $y^{\prime}$ of $Y^{\prime}$, the restriction of $\varphi$ to $e^{\prime}$ is linear with slope (or 'expansion factor') $r_{y^{\prime}}$. Conversely, a finite harmonic morphism of metrized complexes of curves gives rise to an admissible cover of semistable curves (without the supplementary condition on simple ramification) by forgetting the metrics on both sides, remembering only the expansion factor along each edge.

A related but somewhat different Berkovich-theoretic point of view on simultaneous semistable reduction for curves can be found in Welliaveetil's recent preprint [46]; harmonic morphisms of metrized complexes of curves play an implicit role in his paper.

\section{Metric graphs and metrized complexes of curves}

In this section, we recall several definitions of graphs with some additional structures and morphisms between them. A number of these definitions are now standard in tropical geometry; we refer for example to [10,17,35], and [2]. We also provide a number of examples. Some of the definitions in this section are not necessary in the generality or the form in which they are presented for the purposes of this paper, but will be useful in [3].

Throughout this section, we fix $\Lambda$ a non-trivial subgroup of $(\mathbf{R},+)$.

\subsection{Metric graphs}

Given $r \in \mathbf{Z}_{\geq 1}$, we define $S_{r} \subset \mathbf{C}$ to be a 'star with $r$ branches', i.e., a topological space homeomorphic to the convex hull in $\mathbf{R}^{2}$ of $(0,0)$ and any $r$ points, no two of which lie on a common line through the origin. We also define $S_{0}=\{0\}$.

All graphs considered in this paper are assumed to be connected. A finite topological graph $\Gamma$ is the topological realization of a finite graph. That is to say, $\Gamma$ is a compact topological space of dimension at most one (possibly zero) such that for any point $p \in \Gamma$, there exists a neighborhood $U_{p}$ of $p$ in $\Gamma$ homeomorphic to some $S_{r}$; moreover, there are only finitely many points $p$ with $U_{p}$ homeomorphic to $S_{r}$ with $r \neq 2$.

The unique integer $r$ such that $U_{p}$ is homeomorphic to $S_{r}$ is called the valence of $p$ and denoted $\operatorname{val}(p)$. A point of valence different from 2 is called an essential vertex of $\Gamma$. The set of tangent directions at $p$ is $T_{p}(\Gamma)=\lim _{\longrightarrow} U_{p} \pi_{0}\left(U_{p} \backslash\{p\}\right)$, where the limit is taken over all neighborhoods of $p$ isomorphic to a star with $r$ branches. The set $T_{p}(\Gamma)$ has $\operatorname{val}(p)$ elements.

Definition 2.2. A metric graph is a finite connected topological graph $\Gamma$ equipped with a complete inner metric on $\Gamma \backslash V_{\infty}(\Gamma)$, where $V_{\infty}(\Gamma) \subsetneq \Gamma$ is some (finite) set of 1 -valent vertices of $\Gamma$ called infinite vertices of $\Gamma$. (An inner metric is a metric for which the distance between two points $x$ and $y$ is the minimum of the lengths of all paths between $x$ and $y$.) 
Let $\Gamma$ be a metric graph containing a finite essential vertex. We say that $\Gamma$ is a $\Lambda$-metric graph if the distance between any two finite essential vertices of $\Gamma$ lies in $\Lambda$. A $\Lambda$-point of $\Gamma$ is a point of $\Gamma$ whose distance to any finite essential vertex of $\Gamma$ lies in $\Lambda$.

Let $\Gamma$ be a metric graph such that all essential vertices are infinite, so $\Gamma$ is homeomorphic to a circle or a completed line. We say that $\Gamma$ is a $\Lambda$-metric graph if it is a completed line or a circle whose circumference is in $\Gamma$. Given a distinguished point $v \in \Gamma$ of valency equal to 2, a $\Lambda$-point of $\Gamma$ is a point of $\Gamma$ whose distance to $v$ lies in $\Lambda$.

One can equip $\Gamma$ with a degenerate metric in which the infinite vertices are at infinite distance from any other point of $\Gamma$. When no confusion is possible about the subgroup $\Lambda$, we will sometimes write simply metric graph instead of $\Lambda$-metric graph.

Definition 2.3. Let $\Gamma$ be a $\Lambda$-metric graph. A vertex set $V(\Gamma)$ is a finite subset of the $\Lambda$ points of $\Gamma$ containing all essential vertices. An element of a fixed vertex set $V(\Gamma)$ is called a vertex of $\Gamma$, and the closure of a connected component of $\Gamma \backslash V(\Gamma)$ is called an edge of $\Gamma$. An edge which is homeomorphic to a circle is called a loop edge. An edge adjacent to an infinite vertex is called an infinite edge. We denote by $V_{f}(\Gamma)$ the set of finite vertices of $\Gamma$, and by $E_{f}(\Gamma)$ the set of finite edges of $\Gamma$.

Fix a vertex set $V(\Gamma)$. We denote by $E(\Gamma)$ the set of edges of $\Gamma$. Since $\Gamma$ is a metric graph, we can associate to each edge $e$ of $\Gamma$ its length $\ell(e) \in \Lambda \cup\{+\infty\}$. Since the metric on $\Gamma \backslash V_{\infty}(\Gamma)$ is complete, an edge $e$ is infinite if and only if $\ell(e)=+\infty$. The notion of vertices and edges of $\Gamma$ depends, of course, on the choice of a vertex set; we will fix such a choice without comment whenever there is no danger of confusion.

Definition 2.4. Fix vertex sets $V\left(\Gamma^{\prime}\right)$ and $V(\Gamma)$ for two $\Lambda$-metric graphs $\Gamma^{\prime}$ and $\Gamma$, respectively, and let $\varphi: \Gamma^{\prime} \rightarrow \Gamma$ be a continuous map.

- The map $\varphi$ is called a $\left(V\left(\Gamma^{\prime}\right), V(\Gamma)\right)$-morphism of $\Lambda$-metric graphs if we have

(i) $\varphi\left(V\left(\Gamma^{\prime}\right)\right) \subset V(\Gamma)$,

(ii) For each edge $e \in E(\Gamma), \varphi^{-1}(e)$ is a union of edges in $E\left(\Gamma^{\prime}\right)$, and

(iii) the restriction of $\varphi$ to any edge $e^{\prime}$ of $\Gamma^{\prime}$ is a dilation by some factor $d_{e^{\prime}}(\varphi) \in \mathbf{Z}_{\geq 0}$.

If $\Gamma=\{p\}$, we also require the choice of a nonnegative integer $d_{p^{\prime}}(\varphi)$ for each $p^{\prime} \in V_{f}\left(\Gamma^{\prime}\right)$ as part of the data of the morphism $\varphi$. We extend the definition of $d_{p^{\prime}}(\varphi)$ to all $p^{\prime} \in \Gamma^{\prime}$ by setting $d_{p^{\prime}}(\varphi)=0$ for all $p^{\prime} \in \Gamma^{\prime} \backslash V_{f}\left(\Gamma^{\prime}\right)$ (see Remark 2.7).

- The map $\varphi$ is called a morphism of $\Lambda$-metric graphs if there exists a vertex set $V\left(\Gamma^{\prime}\right)$ of $\Gamma^{\prime}$ and a vertex set $V(\Gamma)$ of $\Gamma$ such that $\varphi$ is a $\left(V\left(\Gamma^{\prime}\right), V(\Gamma)\right)$-morphism of $\Lambda$-metric graphs.

- The map $\varphi$ is said to be finite if $d_{e^{\prime}}(\varphi)>0$ for any edge $e^{\prime} \in E\left(\Gamma^{\prime}\right)$.

An edge $e^{\prime}$ of $\Gamma^{\prime}$ is mapped to a vertex of $\Gamma$ if and only if $d_{e^{\prime}}(\varphi)=0$. Such an edge is said to be contracted by $\varphi$. A morphism $\varphi: \Gamma^{\prime} \rightarrow \Gamma$ is finite if and only if there are no contracted edges, which holds if and only if $\varphi^{-1}(p)$ is a finite set for any point $p \in \Gamma$. If $\varphi$ is finite, then $p^{\prime} \in V_{f}\left(\Gamma^{\prime}\right)$ if and only if $\varphi\left(p^{\prime}\right) \in V_{f}(\Gamma)$. 
The integer $d_{e^{\prime}}(\varphi) \in \mathbf{Z}_{\geq 0}$ in Definition 2.4 is called the degree of $\varphi$ along $e^{\prime}$ (it is also sometimes called the weight of $e^{\prime}$ or expansion factor along $e^{\prime}$ in the literature). Since $\ell\left(\varphi\left(e^{\prime}\right)\right)=d_{e^{\prime}}(\varphi) \cdot \ell\left(e^{\prime}\right)$, it follows in particular that if $d_{e^{\prime}}(\varphi) \geq 1$ then $e^{\prime}$ is infinite if and only if $\varphi\left(e^{\prime}\right)$ is infinite. Let $p^{\prime} \in V\left(\Gamma^{\prime}\right)$, let $v^{\prime} \in T_{p^{\prime}}\left(\Gamma^{\prime}\right)$, and let $e^{\prime} \in E\left(\Gamma^{\prime}\right)$ be the edge in the direction of $v^{\prime}$. We define the directional derivative of $\varphi$ in the direction $v^{\prime}$ to be $d_{\nu^{\prime}}(\varphi):=d_{e^{\prime}}(\varphi)$. If we set $p=\varphi\left(p^{\prime}\right)$, then $\varphi$ induces a map

$$
d \varphi\left(p^{\prime}\right):\left\{v^{\prime} \in T_{p^{\prime}}\left(\Gamma^{\prime}\right): d_{\nu^{\prime}}(\varphi) \neq 0\right\} \longrightarrow \rightarrow T_{p}(\Gamma)
$$

in the obvious way.

Example 2.5. Figure 1 depicts four examples of morphisms of metric graphs $\varphi: \Gamma^{\prime} \rightarrow$ $\Gamma$. We use the following conventions in our pictures: black dots represent vertices of $\Gamma^{\prime}$ and $\Gamma$; we label an edge with its degree if and only if the degree is different from 0 and 1 ; we do not specify the lengths of edges of $\Gamma^{\prime}$ and $\Gamma$.

The morphisms depicted in Figure 1a-c are finite, while the one depicted in Figure 1d is not.

Definition 2.6. Let $\varphi: \Gamma^{\prime} \rightarrow \Gamma$ be a morphism of $\Lambda$-metric graphs, let $p^{\prime} \in \Gamma^{\prime}$, and let $p=\varphi\left(p^{\prime}\right)$. The morphism $\varphi$ is harmonic at $p^{\prime}$ provided that, for every tangent direction $v \in T_{p}(\Gamma)$, the number

$$
d_{p^{\prime}}(\varphi):=\sum_{\substack{v^{\prime} \in T_{p^{\prime}}\left(\Gamma^{\prime}\right) \\ v^{\prime} \mapsto v}} d_{\nu^{\prime}}(\varphi)
$$

is independent of $\nu$. The number $d_{p^{\prime}}(\varphi)$ is called the degree of $\varphi$ at $p^{\prime}$. We set $d_{p^{\prime}}(\varphi)=0$ if every edge adjacent to $p^{\prime}$ is contracted, unless $\Gamma=\{p\}$, in which case $d_{p^{\prime}}(\varphi)$ is part of the data of $\varphi$.

We say that $\varphi$ is harmonic if it is surjective and harmonic at all $p^{\prime} \in \Gamma^{\prime}$; in this case, since $\Gamma$ is connected, the number $\operatorname{deg}(\varphi)=\sum_{p^{\prime} \mapsto p} d_{p^{\prime}}(\varphi)$ is independent of $p \in \Gamma$ and is called the degree of $\varphi$.

Remark 2.7. In the above situation, if all edges adjacent to a vertex $p^{\prime} \in V\left(\Gamma^{\prime}\right)$ are contracted, then $\varphi$ is automatically harmonic at $p^{\prime}$. In particular, if $\Gamma$ consists of a single

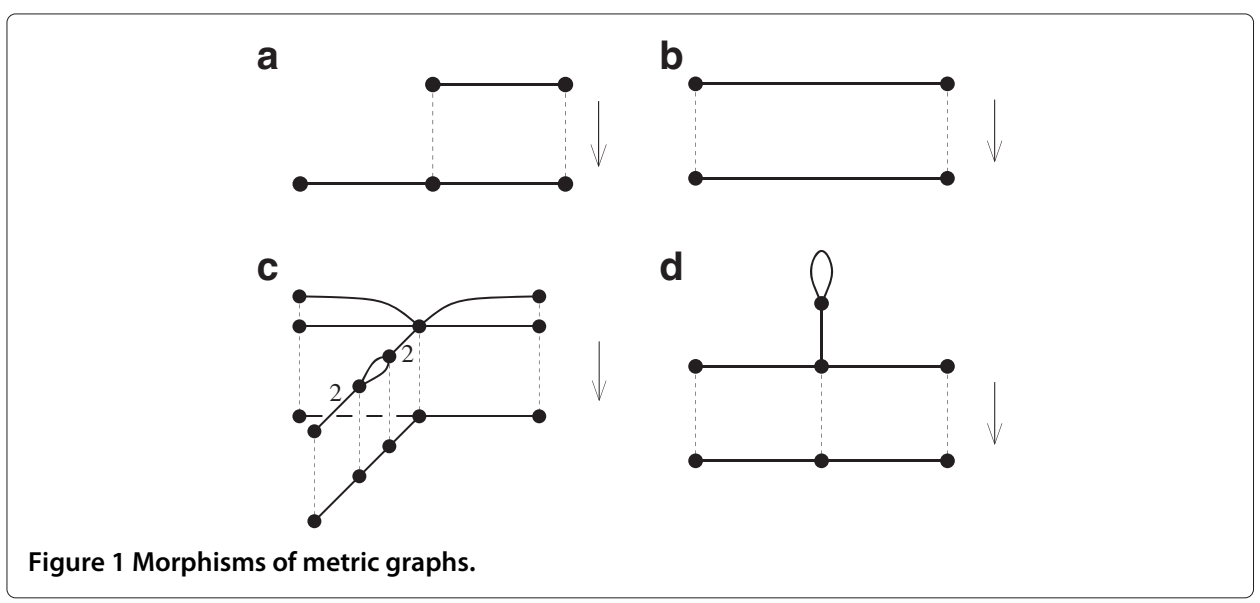


vertex $p$ and $\varphi: \Gamma^{\prime} \rightarrow \Gamma$ is a morphism of metric graphs, then $\varphi$ is by definition harmonic. In this case, we have $\operatorname{deg}(\varphi)=\sum_{p^{\prime} \in \Gamma^{\prime}} d_{p^{\prime}}(\varphi)$, and if $\varphi$ is finite, then $\Gamma^{\prime}=\left\{p^{\prime}\right\}$, in which case $\operatorname{deg}(\varphi)=d_{p^{\prime}}(\varphi)$.

If both $\Gamma^{\prime}$ and $\Gamma$ have at least one edge, then a non-constant morphism which is harmonic at all $p^{\prime} \in V\left(\Gamma^{\prime}\right)$ is automatically surjective.

Example 2.8. The morphism depicted in Figure 1a is not harmonic, while the ones depicted in Figure 1b-d are (for suitable choices of lengths).

\subsection{Harmonic morphisms and harmonic functions}

Given a metric graph $\Gamma$ and a non-empty open set $U$ in $\Gamma$, a function $f: U \rightarrow \mathbf{R}$ is said to be harmonic on $U$ if $f$ is piecewise affine with integer slopes and for all $x \in U$, the sum of the slopes of $f$ along all outgoing tangent directions at $x$ is equal to 0 .

Since we will not use it elsewhere in the paper, we omit the proof of the following result (which is very similar to the proof of [8], Proposition 2.6):

Proposition 2.10. A surjective morphism $\varphi: \Gamma^{\prime} \rightarrow \Gamma$ of metric graphs is harmonic if and only if for every open set $U \subseteq \Gamma$ and every harmonic function $f: U \rightarrow \mathbf{R}$, the pullback function $\varphi^{*} f: \varphi^{-1}(U) \rightarrow \mathbf{R}$ is also harmonic.

Equivalently, a morphism of metric graphs is harmonic if and only if germs of harmonic functions pullback to germs of harmonic functions. For a metric graph $\Gamma$, we let $\operatorname{Div}(\Gamma)$ denote the free abelian group on $\Gamma$. Given a harmonic morphism $\varphi: \Gamma^{\prime} \rightarrow \Gamma$ of metric graphs, there are natural pullback and push-forward homomorphisms $\varphi^{*}: \operatorname{Div}(\Gamma) \rightarrow$ $\operatorname{Div}\left(\Gamma^{\prime}\right)$ and $\varphi_{*}: \operatorname{Div}\left(\Gamma^{\prime}\right) \rightarrow \operatorname{Div}(\Gamma)$ defined by

$$
\varphi^{*}(p)=\sum_{p^{\prime} \mapsto p} d_{p^{\prime}}(\varphi)\left(p^{\prime}\right) \quad \text { and } \quad \varphi_{*}\left(p^{\prime}\right)=\left(\varphi\left(p^{\prime}\right)\right)
$$

and extending linearly. It is clear that for $D \in \operatorname{Div}(\Gamma)$, we have $\operatorname{deg}\left(\varphi_{*}(D)\right)=\operatorname{deg}(D)$ and $\operatorname{deg}\left(\varphi^{*}(D)\right)=\operatorname{deg}(\varphi) \cdot \operatorname{deg}(D)$.

\subsection{Augmented metric graphs}

Here, we consider $\Lambda$-metric graphs together with the data of a non-negative integer at each point. For a finite point $p$, this integer should be thought as the genus of a 'virtual algebraic curve' lying above $p$.

Definition 2.13. An augmented $\Lambda$-metric graph is a $\Lambda$-metric graph $\Gamma$ along with a function $g: \Gamma \rightarrow \mathbf{Z}_{\geq 0}$, called the genus function, such that $g(p)=0$ for all points of $\Gamma$ except possibly for finitely many (finite) $\Lambda$-points $p \in \Gamma$. The essential vertices of $\Gamma$ are the points $p$ for which $\operatorname{val}(p) \neq 2$ or $g(p)>0$. A vertex set of $\Gamma$ is a vertex set $V(\Gamma)$ of the underlying metric graph which contains all essential vertices of $\Gamma$ as an augmented graph.

An augmented metric graph is said to be totally degenerate if the genus function is identically zero. 
The genus of $\Gamma$ is defined to be

$$
g(\Gamma)=h_{1}(\Gamma)+\sum_{p \in \Gamma} g(p)
$$

where $h_{1}(\Gamma)=\operatorname{dim}_{\mathbf{Q}} H_{1}(\Gamma, \mathbf{Q})$ is the first Betti number of $\Gamma$. The canonical divisor on $\Gamma$ is

$$
K_{\Gamma}=\sum_{p \in \Gamma}(\operatorname{val}(p)+2 g(p)-2)(p)
$$

A harmonic morphism of augmented $\Lambda$-metric graphs $\varphi: \Gamma^{\prime} \rightarrow \Gamma$ is a map which is a harmonic morphism between the underlying metric graphs of $\Gamma^{\prime}$ and $\Gamma$.

Note that both summations in the above definition are in fact over essential vertices of $\Gamma$. The degree of the canonical divisor of an augmented $\Lambda$-metric graph $\Gamma$ is $\operatorname{deg}\left(K_{\Gamma}\right)=$ $2 g(\Gamma)-2$. An augmented metric graph of genus 0 will also be called a rational augmented metric graph. Let $\varphi: \Gamma^{\prime} \rightarrow \Gamma$ be a harmonic morphism of augmented $\Lambda$-metric graphs. The ramification divisor of $\varphi$ is the divisor $R=\sum R_{p^{\prime}}\left(p^{\prime}\right)$, where

$$
R_{p^{\prime}}=d_{p^{\prime}}(\varphi) \cdot\left(2-2 g\left(\varphi\left(p^{\prime}\right)\right)\right)-\left(2-2 g\left(p^{\prime}\right)\right)-\sum_{v^{\prime} \in T_{p^{\prime}}\left(\Gamma^{\prime}\right)}\left(d_{v^{\prime}}(\varphi)-1\right) .
$$

If $p^{\prime}$ is a non-essential vertex of $\Gamma^{\prime}$, then one verifies by direct computation that $R_{p^{\prime}}=0$ if $d_{p^{\prime}}(\varphi)=0$ or if $p=\varphi\left(p^{\prime}\right)$ is a non-essential vertex of $\Gamma$. In particular, the sum $\sum R_{p^{\prime}}\left(p^{\prime}\right)$ is in fact finite, so $R$ is well-defined. Note that if $p^{\prime} \in V_{\infty}\left(\Gamma^{\prime}\right)$ and $d_{p^{\prime}}(\varphi)>0$, then $\varphi\left(p^{\prime}\right)$ is also an infinite vertex and $R_{p^{\prime}} \geq 0$, whereas if $d_{p^{\prime}}(\varphi)=0$, then $R_{p^{\prime}}=-1$.

The definition of $R_{v^{\prime}}$ in Equation 2.14.1 is rigged so that we have the following graphtheoretic analogue of the Riemann-Hurwitz formula:

$$
K_{\Gamma^{\prime}}=\varphi^{*}\left(K_{\Gamma}\right)+R
$$

Definition 2.15. Let $\varphi: \Gamma^{\prime} \rightarrow \Gamma$ be a finite harmonic morphism of augmented $\Lambda$ metric graphs. We say that $\varphi$ is étale at a point $p^{\prime} \in \Gamma^{\prime}$ provided that $R_{p^{\prime}}=0$. We say that $\varphi$ is generically étale if $R$ is supported on the set of infinite vertices of $\Gamma^{\prime}$ and that $\varphi$ is étale if $R=0$.

\subsection{Metrized complexes of curves}

Recall that $k$ is an algebraically closed field. A metrized complex of curves is, roughly speaking, an augmented metric graph $\Gamma$ together with a chosen vertex set $V(\Gamma)$ and a marked algebraic $k$-curve of genus $g(p)$ for each finite vertex $p \in V(\Gamma)$. More precisely:

Definition 2.17. A $\Lambda$-metrized complex of $k$-curves consists of the following data:

- An augmented $\Lambda$-metric graph $\Gamma$ equipped with the choice of a distinguished vertex set $V(\Gamma)$.

- For every finite vertex $p$ of $\Gamma$, a smooth, proper, connected $k$-curve $C_{p}$ of genus $g(p)$. 
- An injective function $\operatorname{red}_{p}: T_{p}(\Gamma) \rightarrow C_{p}(k)$, called the reduction map. We call the image of $\operatorname{red}_{p}$ the set of marked points on $C_{p}$.

Example 2.18. Figure 2 depicts a particular metrized complex of curves over $\mathbf{C}$. At each finite vertex $p$ there is an associated compact Riemann surface $C_{p}$, together with a finite set of marked points (in red).

Definition 2.19. A harmonic morphism of metrized complexes of curves consists of a harmonic $\left(V\left(\Gamma^{\prime}\right), V(\Gamma)\right)$-morphism $\varphi: \Gamma^{\prime} \rightarrow \Gamma$ of augmented metric graphs, and for every finite vertex $p^{\prime}$ of $\Gamma^{\prime}$ with $d_{p^{\prime}}(\varphi)>0$, a finite morphism of curves $\varphi_{p^{\prime}}: C_{p^{\prime}}^{\prime} \rightarrow C_{\varphi\left(p^{\prime}\right)}$, satisfying the following compatibility conditions:

1. For every finite vertex $p^{\prime} \in V\left(\Gamma^{\prime}\right)$ and every tangent direction $v^{\prime} \in T_{p^{\prime}}\left(\Gamma^{\prime}\right)$ with $d_{v^{\prime}}(\varphi)>0$, we have $\varphi_{p^{\prime}}\left(\operatorname{red}_{p^{\prime}}\left(v^{\prime}\right)\right)=\operatorname{red}_{\varphi\left(p^{\prime}\right)}\left(d \varphi\left(p^{\prime}\right)\left(v^{\prime}\right)\right)$, and the ramification degree of $\varphi_{p^{\prime}}$ at $\operatorname{red}_{p^{\prime}}\left(v^{\prime}\right)$ is equal to $d_{v^{\prime}}(\varphi)$.

2. For every finite vertex $p^{\prime} \in V\left(\Gamma^{\prime}\right)$ with $d_{p^{\prime}}(\varphi)>0$, every tangent direction $v \in T_{\varphi\left(p^{\prime}\right)}(\Gamma)$, and every point $x^{\prime} \in \varphi_{p^{\prime}}^{-1}\left(\operatorname{red}_{\varphi\left(p^{\prime}\right)}(v)\right) \subset C_{p^{\prime}}^{\prime}(k)$, there exists $v^{\prime} \in T_{p^{\prime}}\left(\Gamma^{\prime}\right)$ such that $\operatorname{red}_{p^{\prime}}\left(v^{\prime}\right)=x^{\prime}$.

3. For every finite vertex $p^{\prime} \in V\left(\Gamma^{\prime}\right)$ with $d_{p^{\prime}}(\varphi)>0$, we have $d_{p^{\prime}}(\varphi)=\operatorname{deg}\left(\varphi_{p^{\prime}}\right)$.

Note that we do not require the morphisms $\varphi_{p^{\prime}}: C_{p^{\prime}}^{\prime} \rightarrow C_{p}$ to be ramified only at the marked points corresponding to the tangent directions at $p^{\prime}$ on the metric graph $\Gamma^{\prime}$; see however Proposition 2.22 which naturally guarantees this property.

A harmonic morphism of metrized complexes of curves is called finite if the underlying harmonic morphism of (augmented) metric graphs is finite. Metrized complexes of curves with harmonic morphisms between them form a category, with finite harmonic morphisms giving rise to a subcategory.

If $\Gamma$ has at least one edge, then (3) follows from (1) and (2), since the sum of the ramification degrees of a finite morphism of curves along any fiber is equal to the degree of the morphism.

Let $\varphi: C^{\prime} \rightarrow C$ be a finite morphism of smooth, proper, connected $k$-curves. Recall that $\varphi$ is tamely ramified if either $\operatorname{char}(k)=0$ or the ramification degree of $\varphi$ at every closed point of $C^{\prime}$ is prime to the characteristic of $k$.

\section{Remark 2.20.}

1. Let $\varphi: C^{\prime} \rightarrow C$ be a finite morphism. Let $D$ be the (finite) set of branch points, let $D^{\prime}=\varphi^{-1}(D) \subset C^{\prime}(k)$, and let $U=C \backslash D$ and $U^{\prime}=C^{\prime} \backslash D^{\prime}$. Then $\varphi$ is tamely

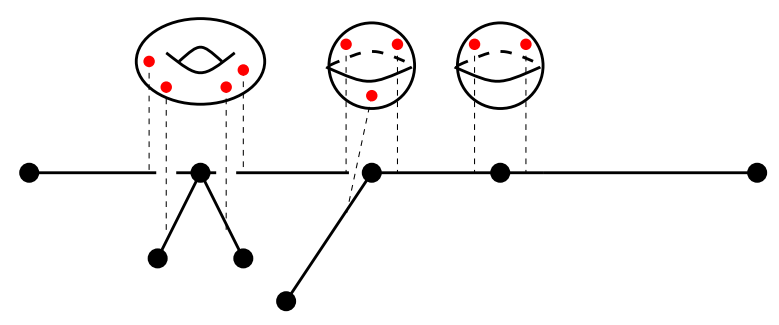

Figure 2 Metrized complex of curves over $\mathbf{C}$. 
ramified if and only if $\left.\varphi\right|_{U^{\prime}}: U^{\prime} \rightarrow U$ is a tamely ramified cover of $U$ over $C$ relative to $D$ in the sense of ([40], XIII.2.1.3): see the discussion in $\S 2.0$ of loc. cit.

2. A tamely ramified morphism is separable (i.e. generically étale).

3. The Riemann-Hurwitz formula applies to a tamely ramified morphism.

Definition 2.21. Let $\varphi: \mathcal{C}^{\prime} \rightarrow \mathcal{C}$ be a finite harmonic morphism of metrized complexes of curves. We say that $\varphi$ is a tame harmonic morphism if either $\operatorname{char}(k)=0$, or $\operatorname{char}(k)=$ $p>0$ and $\varphi_{p^{\prime}}$ is tamely ramified for all finite vertices $p^{\prime} \in V\left(\Gamma^{\prime}\right)$. We call $\varphi$ a tame covering if in addition $\varphi: \Gamma^{\prime} \rightarrow \Gamma$ is a generically étale finite morphism of augmented metric graphs.

Let $\varphi: \mathcal{C}^{\prime} \rightarrow \mathcal{C}$ be a tame harmonic morphism. Then, each $\varphi_{p^{\prime}}: C_{p^{\prime}} \rightarrow C_{\varphi\left(p^{\prime}\right)}$ is a separable morphism. Note that if $\operatorname{char}(k)=p$, then $d_{e^{\prime}}(\varphi)$ is prime to $p$ for all edges $e^{\prime}$ of $\Gamma^{\prime}$. We have the following kind of converse statement:

Proposition 2.22. Let $\varphi: \mathcal{C}^{\prime} \rightarrow \mathcal{C}$ be a finite harmonic morphism of metrized complexes of curves. Suppose that $\varphi$ is generically étale and that $\varphi_{p^{\prime}}: C_{p^{\prime}} \rightarrow C_{p}$ is a separable morphism of curves for all finite vertices $p^{\prime}$ of $\Gamma^{\prime}$. Let $p^{\prime}$ be a finite vertex of $\Gamma^{\prime}$, and suppose that $\varphi_{p^{\prime}}$ is ramified at a point $x^{\prime} \in C_{p^{\prime}}^{\prime}(k)$. Then, there exists $v^{\prime} \in T_{p^{\prime}}\left(\Gamma^{\prime}\right)$ such that red $d_{p^{\prime}}\left(v^{\prime}\right)=x^{\prime}$. Moreover, $\varphi_{p^{\prime}}$ is automatically tamely ramified and $\varphi$ is a tame covering.

Proof. Let $p=\varphi\left(p^{\prime}\right)$. Since $\varphi$ is generically étale, we have

$$
0=R_{p^{\prime}}=d_{p^{\prime}}(\varphi)(2-2 g(p))-\left(2-2 g\left(p^{\prime}\right)\right)-\sum_{v^{\prime} \in T_{p^{\prime}}\left(\Gamma^{\prime}\right)}\left(d_{\nu^{\prime}}(\varphi)-1\right) .
$$

Since $g(p)=g\left(C_{p}\right), g\left(p^{\prime}\right)=g\left(C_{p^{\prime}}^{\prime}\right)$, and $d_{p^{\prime}}(\varphi)$ is the degree of $\varphi_{p^{\prime}}: C_{p^{\prime}}^{\prime} \rightarrow C_{p}$, this gives

$$
\sum_{v^{\prime} \in T_{p^{\prime}}\left(\Gamma^{\prime}\right)}\left(d_{\nu^{\prime}}(\varphi)-1\right)=\operatorname{deg}\left(\varphi_{p^{\prime}}\right)\left(2-2 g\left(C_{p}\right)\right)-\left(2-2 g\left(C_{p^{\prime}}^{\prime}\right)\right) .
$$

The Riemann-Hurwitz formula as applied to $\varphi_{p^{\prime}}$ says that the right-hand side of the above equation is equal to the degree of the ramification divisor of $\varphi_{p^{\prime}}$ (this holds whenever $\varphi_{p^{\prime}}$ is separable). For all $v^{\prime} \in T_{p^{\prime}}\left(\Gamma^{\prime}\right)$, the ramification degree $e_{x^{\prime}}$ of $\varphi_{p^{\prime}}$ at $x^{\prime}=\operatorname{red}_{p^{\prime}}\left(v^{\prime}\right)$ is $d_{\nu^{\prime}}(\varphi)$, so we have an equality between the degree of the ramification divisor of $\varphi_{p^{\prime}}$ and $\sum_{x^{\prime}=\operatorname{red}_{p^{\prime}}\left(v^{\prime}\right)}\left(e_{x^{\prime}}-1\right)$. This implies both that any ramification point $x^{\prime} \in C_{p^{\prime}}^{\prime}(k)$ is the reduction of a tangent direction, and in addition that the contribution of $x^{\prime}$ to the ramification divisor of $\varphi_{p^{\prime}}$ is equal to $e_{x^{\prime}}-1$, from which it follows that $\varphi_{p^{\prime}}$ is tamely ramified at $x^{\prime}$.

Remark 2.23. Loosely speaking, Proposition 2.22 says that all ramification of all morphisms $\varphi_{p^{\prime}}$ is 'visible' in the edge degrees of the underlying morphism of metric graphs. It follows from Proposition 2.22 that if $\Gamma^{\prime}=\left\{p^{\prime}\right\}$, then $\varphi_{p^{\prime}}$ is étale.

\section{Analytic curves and their skeleta}

In this section, we recall the definition and basic properties of the skeleton of the analytification of an algebraic curve as outlined in [43] and elaborated in [12]. These foundational results are well known to experts and appear in various guises in the literature. See, for example, Berkovich [14,16], Thuillier [44], and Ducros [25,26]. In this section, we also 
show that the skeleton is naturally a metrized complex of curves and prove that any metrized complex arises as the skeleton of a curve. Finally, we introduce the important notion of a triangulated punctured curve, which is essentially a punctured curve along with the data of a skeleton with a distinguished set of vertices.

Recall that $K$ is an algebraically closed field which is complete with respect to a nontrivial non-Archimedean valuation val $: K \rightarrow \mathbf{R} \cup\{\infty\}$. Its valuation ring is $R$, its maximal ideal is $\mathfrak{m}_{R}$, its residue field is $k=R / \mathfrak{m}_{R}$, and its value group is $\Lambda=\operatorname{val}\left(K^{\times}\right) \subset \mathbf{R}$. The assumption that $K$ is algebraically closed is mostly for simplicity, to avoid complications due to Galois actions on non-split semistable models and nontrivial twists of analytic balls and annuli (see however Section 5 where we explain how to use descent arguments to remove this restriction and get results over more general ground fields). We assume that $K$ is nontrivially valued because the theory is mostly tautological in the trivially valued case.

By an analytic curve, we mean a strictly $K$-analytic space $X$ of pure dimension 1 . For our purposes, the most important example of an analytic curve is the analytification of a smooth, connected, projective algebraic $K$-curve. If $X$ is an analytic curve, then we define $\mathbf{H}(X)=X \backslash X(K)$ as in ([12], §5).

\subsection{Analytic building blocks}

We identify the set underlying the analytification of the affine line with the set of multiplicative seminorms $\|\cdot\|: K[t] \rightarrow \mathbf{R}_{\geq 0}$ which extend the absolute value on $K$. We let val : $\mathbf{A}^{1 \text {,an }} \rightarrow \mathbf{R} \cup\{\infty\}$ denote the valuation map

$$
\operatorname{val}(x)=-\log |x|=-\log \|t\|_{x} .
$$

For $a \in K^{\times}$, the standard closed ball of radius $|a|$ is the affinoid domain

$$
\mathbf{B}(a)=\operatorname{val}^{-1}([\operatorname{val}(a), \infty]) \subset \mathbf{A}^{1, \mathrm{an}}
$$

and the standard open ball of radius $|a|$ is the open analytic domain

$$
\mathbf{B}(a)_{+}=\operatorname{val}^{-1}((\operatorname{val}(a), \infty]) \subset \mathbf{A}^{1, \mathrm{an}} .
$$

Note that scaling by $a$ gives isomorphisms $\mathbf{B}(1) \stackrel{\sim}{\longrightarrow} \mathbf{B}(a)$ and $\mathbf{B}(1)_{+} \stackrel{\sim}{\longrightarrow} \mathbf{B}(a)_{+}$. We call $\mathbf{B}(1)$ (resp. $\left.\mathbf{B}(1)_{+}\right)$the standard closed (resp. open) unit ball. For $a \in K^{\times}$with val $(a) \geq$ 0 , the standard closed annulus of modulus $\operatorname{val}(a)$ is the affinoid domain

$$
\mathbf{S}(a)=\operatorname{val}^{-1}([0, \operatorname{val}(a)]) \subset \mathbf{A}^{1, \text { an }}
$$

and if $\operatorname{val}(a)>0$, the standard open annulus of modulus $\operatorname{val}(a)$ is the open analytic domain

$$
\mathbf{S}(a)_{+}=\operatorname{val}^{-1}((0, \operatorname{val}(a))) \subset \mathbf{A}^{1, \text { an }} .
$$

The standard punctured open ball is the open analytic domain

$$
\mathbf{S}(0)_{+}=\mathrm{val}^{-1}((0, \infty))=\mathbf{B}(1)_{+} \backslash\{0\} \subset \mathbf{A}^{1, \mathrm{an}} .
$$

By a closed unit ball (resp. open unit ball, resp. closed annulus, resp. open annulus, resp. punctured open ball), we will mean a $K$-analytic space which is isomorphic to the standard closed unit ball (resp. the standard open unit ball, resp. the standard closed annulus 
of modulus val $(a)$ for some $a \in R \backslash\{0\}$, resp. the standard open annulus of modulus val( $a$ ) for some $a \in \mathfrak{m}_{R} \backslash\{0\}$, resp. the standard punctured open ball). A generalized open annulus is a $\mathrm{K}$-analytic space which is either an open annulus or a punctured open ball. It is a standard fact that the modulus of a closed (resp. open) annulus is an isomorphism invariant; see for instance [12], Corollary 2.6. We define the modulus of a punctured open ball to be $\infty$.

Let $a \in \mathfrak{m}_{R}$ (so $a=0$ is allowed). There is a natural section $\sigma:(0, \operatorname{val}(a)) \rightarrow \mathbf{S}(a)_{+}$of the valuation map val : $\mathbf{S}(a)_{+} \rightarrow(0, \operatorname{val}(a))$ sending $r$ to the maximal point of the affinoid domain $\mathrm{val}^{-1}(r)$ if $r \in \Lambda$, resp. the only point of $\mathrm{val}^{-1}(r)$ if $r \notin \Lambda$. The skeleton of $\mathbf{S}(a)_{+}$ is defined to be the image of $\sigma$ and is denoted $\Sigma\left(\mathbf{S}(a)_{+}\right)=\sigma((0, \operatorname{val}(a)))$. We will always identify the skeleton of the standard generalized open annulus $\mathbf{S}(a)_{+}$with the interval or ray $(0, \operatorname{val}(a))$. It follows from [12], Proposition 2.5, that the skeleton of a standard open annulus or standard punctured open ball is an isomorphism invariant, so if $A$ is a generalized open annulus, then we can define the skeleton $\Sigma(A)$ of $A$ to be the image of the skeleton of a standard open annulus or standard punctured open ball $\mathbf{S}(a)_{+}$under any isomorphism $\mathbf{S}(a)_{+} \stackrel{\sim}{\longrightarrow} A$.

Let $T$ be a metric space and let $x, y \in T$. A geodesic segment from $x$ to $y$ is the image of a locally isometric embedding $[a, b] \hookrightarrow T$ with $a \mapsto x$ and $b \mapsto y$. We often identify a geodesic segment with its image in $T$. Recall that an $\mathbf{R}$-tree is a metric space $T$ with the following properties:

(i) For all $x, y \in T$, there is a unique geodesic segment $[x, y]$ from $x$ to $y$.

(ii) For all $x, y, z \in T$, if $[x, y] \cap[y, z]=\{y\}$, then $[x, z]=[x, y] \cup[y, z]$.

See [9], Appendix B. It is proved in $\S 1.4$ of loc. cit. that $\mathbf{H}(\mathbf{B}(1))$ is naturally an $\mathbf{R}$ tree. (Note however that the topology induced by the $\mathbf{R}$-tree structure is finer than the analytic topology.) Since any path-connected subspace of an $\mathbf{R}$-tree is an $\mathbf{R}$-tree as well, if $X$ is a standard open annulus or standard (punctured) open ball, then $\mathbf{H}(X)$ is an $\mathbf{R}$-tree. It is proved in [12], Corollary 5.6, that the metric structure on $\mathbf{H}(X)$ is an isomorphism invariant, so the same statement applies to open balls and generalized open annuli. For $a \in \mathfrak{m}_{R}$, the section $\sigma:(0, \operatorname{val}(a)) \rightarrow \mathbf{S}(a)_{+}$is an isometry.

It also follows from the results in [9], §1.4, that for any $x \in \mathbf{H}(\mathbf{B}(1))$ and any type-1 point $y \in \mathbf{B}(1)$, there is a unique continuous injection $\alpha:[0, \infty] \hookrightarrow \mathbf{B}(1)$ with $\alpha(0)=x$ and $\alpha(\infty)=y, \alpha([0, \infty)) \subset \mathbf{H}(\mathbf{B}(1))$, and such that $\alpha$ is an isometry when restricted to $[0, \infty)$. We let $[x, y]$ denote the image of $\alpha$, and we call $[x, y]$ the geodesic segment from $x$ to $y$. Similarly, if $x$ and $y$ are both type- 1 points, then there is a unique continuous injection $\alpha:[-\infty, \infty] \rightarrow \mathbf{B}(1)$ with $\alpha(-\infty)=x$ and $\alpha(\infty)=y, \alpha(\mathbf{R}) \subset \mathbf{H}(\mathbf{B}(1))$, and such that $\left.\alpha\right|_{\mathbf{R}}$ is an isometry; the image of $\alpha$ is called the geodesic segment from $x$ to $y$ and is denoted $[x, y]$. Restricting to a suitable analytic subdomain of $\mathbf{B}(1)$ allows us to define geodesic segments between any two points of an open ball or generalized open annulus.

\subsection{Open balls}

The closure of $\mathbf{B}(1)_{+}$in $\mathbf{B}(1)$ consists of $\mathbf{B}(1)_{+}$and a single type- 2 point $x$, called the end of $\mathbf{B}(1)_{+}$. The end is the Shilov boundary point of $\mathbf{B}(1)$ : see for instance the proof of [12], Lemma 3.2. The valuation map val : $\mathbf{B}(1)_{+} \rightarrow(0, \infty]$ extends to a continuous map val : $\mathbf{B}(1)_{+} \cup\{x\} \rightarrow[0, \infty]$. The set $\mathbf{B}(1)_{+} \cup\{x\}$ is path-connected and compact, being a closed subspace of the compact space $\mathbf{B}(1)$. (In fact $\mathbf{B}(1)_{+} \cup\{x\}$ is the one-point 
compactification of $\left.\mathbf{B}(1)_{+}.\right)$For any $y \in \mathbf{B}(1)_{+}$, the geodesic segment $[x, y] \subset \mathbf{B}(1)$ is contained in $\mathbf{B}(1)_{+} \cup\{x\}$.

Lemma 3.3. Let $X=\operatorname{Spec}(A)$ be an irreducible affine $K$-curve, and let $\varphi: \mathbf{B}(1)_{+} \rightarrow X^{\text {an }}$ be a morphism with finite fibers. Then, $\varphi$ extends in a unique way to a continuous map $\mathbf{B}(1)_{+} \cup\{x\} \rightarrow X^{a n}$, and the image of $x$ is a type-2 point of $X^{a n}$.

Proof. Let $f \in A$ be nonzero and define $F:(0, \infty) \rightarrow \mathbf{R}$ by $F(r)=-\log \mid f \circ$ $\varphi(\sigma(r)) \mid$, where $\sigma:(0, \infty) \rightarrow \mathbf{S}(0)_{+} \subset \mathbf{B}(1)_{+}$is the canonical section of val. By [12], Proposition 2.10, $F$ is a piecewise affine function which is differentiable away from finitely many points $r \in \Lambda \cap(0, \infty)$, and for any point $r \in(0, \infty)$ at which $F$ is differentiable, the derivative of $F$ is equal to the number of zeros $y$ of $f \circ \varphi$ with $\operatorname{val}(y)>r$ (note that $f \circ \varphi$ has finitely many zeros since $f$ is algebraic and $\varphi$ has finite fibers). It follows that $F(\Lambda \cap(0, \infty)) \subset \Lambda$. Since $f \circ \varphi$ has finitely many zeros, the limit $\lim _{r \rightarrow 0} F(r)$ exists and is contained in $\Lambda$. We define $\|f\|_{0}=\exp \left(-\lim _{r \rightarrow 0} F(r)\right)$. It is easy to see that $f \mapsto\|f\|_{0}$ is a multiplicative seminorm on $A$ extending the absolute value on $K$, so $\|\cdot\|_{0}$ is a point in $X^{\text {an }}$. Define $\varphi(x)=\|\cdot\|_{0}$. One shows as in the proof of [12], Lemma 3.2, that $\varphi$ is continuous on $\mathbf{B}(1)_{+} \cup\{x\}$.

It remains to show that $\varphi(x)=\|\cdot\|_{0}$ is a type-2 point of $X^{\text {an }}$. Let $f \in A$ be a nonconstant function that has a zero on $\varphi\left(\mathbf{B}(1)_{+}\right)$. Since $-\log \|f\|_{0} \in \Lambda$, there exists $\alpha \in K^{\times}$ such that $\|\alpha f\|_{0}=1$; replacing $f$ by $\alpha f$, we may and do assume that $\|f\|_{0}=1$. Since $\varphi \circ f$ has a zero, by the above we have that $F=-\log |f \circ \varphi \circ \sigma|$ is monotonically increasing, so $F(r)>0$ for all $r \in(0, \infty)$. For any $r \in(0, \infty)$, the maximal point of $\mathrm{val}^{-1}(r) \subset \mathbf{B}(1)_{+}$is equal to $\sigma(r)$, so for any $y \in \mathbf{B}(1)+\operatorname{such}$ that $\operatorname{val}(y)=r$, we have $F(r) \leq-\log |f \circ \varphi(y)|$. It follows that $|f \circ \varphi(y)|<1$ for all $y \in \mathbf{B}(1)_{+}$, so $\varphi\left(f\left(\mathbf{B}(1)_{+}\right)\right) \subset \mathbf{B}(1)_{+}$. Since $\mathbf{B}(1)_{+}$is dense in $\mathbf{B}(1)_{+} \cup\{x\}$, the image of $x$ under $\varphi \circ f$ is contained in the closure $\mathbf{B}(1)_{+} \cup\{x\}$ of $\mathbf{B}(1)_{+}$in $\mathbf{B}(1)$. Since $1=\|f\|_{0}=|f \circ \varphi(x)|$, the point $f \circ \varphi(x) \notin \mathbf{B}(1)_{+}$, so $f \circ \varphi(x)=x$, which is a type-2 point of $\mathbf{A}^{1 \text {,an }}$. Therefore, $\varphi(x)$ is a type- 2 point of $X^{\text {an }}$.

Applying Lemma 3.3 to a morphism $\varphi: \mathbf{B}(1)_{+} \rightarrow \mathbf{B}(1)_{+} \subset \mathbf{A}^{1 \text {,an }}$, we see that any automorphism of $\mathbf{B}(1)_{+}$extends (uniquely) to a homeomorphism $\mathbf{B}(1)_{+} \cup\{x\} \rightarrow \mathbf{B}(1)_{+} \cup$ $\{x\}$, so it makes sense to speak of the end of any open ball. (One can also see this from the fact that $\mathbf{B}(1)_{+} \cup\{x\}$ is the one-point compactification of $\left.\mathbf{B}(1)_{+}.\right)$If $B$ is an open ball with end $x$, we let $\bar{B}$ denote $B \cup\{x\}$.

Let $B$ be an open ball with end $x$. We define a partial ordering on $\bar{B}$ by declaring $y \leq z$ if $y \in[x, z]$; again see ([9], §1.4). Equivalently, for $y, z \in B$ we have $y \leq z$ if and only if $|f(y)| \geq|f(z)|$ for all analytic functions $f$ on $B$. The following lemma is proved as in ([12], Proposition 3.13).

Lemma 3.4. Let $B$ be an open ball, and let $y \in B$ be a type-2 point. Then, $B \backslash\{y\}$ is a disjoint union of the open annulus $A=\{z \in B: z \nsucceq y\}$ with infinitely many open balls, and $B_{y}=\{z \in B: z \geq y\}$ is an affinoid subdomain of $B$ isomorphic to the closed ball $\mathbf{B}(1)$.

\subsection{Open annuli}

Let $a \in \mathfrak{m}_{R} \backslash\{0\}$. The closure of $\mathbf{S}(a)_{+}$in $\mathbf{B}(1)$ consists of $\mathbf{S}(a)_{+}$and the two type-2 points $x=\sigma(0)$ and $y=\sigma(\operatorname{val}(a))$, called the ends of $\mathbf{S}(a)_{+}$: again see the proof of [12], 
Lemma 3.2. The valuation map val $: \mathbf{S}(a)_{+} \rightarrow(0, \operatorname{val}(a))$ extends to a continuous map $\mathrm{val}: \mathbf{S}(a)_{+} \cup\{x, y\} \rightarrow[0, \operatorname{val}(a)]$, and for any $z \in \mathbf{S}(a)_{+}$, the geodesic segments $[x, z]$ and $[y, z]$ are contained in $\mathbf{S}(a)_{+} \cup\{x, y\}$. The following lemma is proved in the same way as the first part of Lemma 3.3.

Lemma 3.6. Let $X=\operatorname{Spec}(A)$ be an irreducible affine $K$-curve, let $a \in \mathfrak{m}_{R} \backslash\{0\}$, and let $\varphi: \mathbf{S}(a)_{+} \rightarrow X^{a n}$ be a morphism with finite fibers. Let $x=\sigma(0)$ and $y=\sigma(\operatorname{val}(a))$ be the ends of $\mathbf{S}(a)_{+}$. Then, $\varphi$ extends in a unique way to a continuous map $\mathbf{S}(a)_{+} \cup\{x, y\} \rightarrow X^{a n}$.

It follows from Lemma 3.6 that any automorphism of $\mathbf{S}(a)_{+}$extends to a homeomorphism $\mathbf{S}(a)_{+} \cup\{x, y\} \stackrel{\sim}{\longrightarrow} \mathbf{S}(a)_{+} \cup\{x, y\}$, so it makes sense to speak of the ends of any open annulus. Alternatively, one can use the fact that the ends as defined here are precisely the canonical endpoints in the terminology of the theory of $\mathbf{R}$-trees (see e.g. [14] or [25], Chapter 1). If $A$ is an open annulus with ends $x, y$, then we let $\bar{A}$ denote the compact space $A \cup\{x, y\}$.

The closure of the punctured open ball $\mathbf{S}(0)_{+}$in $\mathbf{B}(1)$ is equal to $\mathbf{S}(0)_{+} \cup\{0, x\}$, where $x$ is the end of $\mathbf{B}(1)_{+}$. We define $x$ to be the end of $\mathbf{S}(0)_{+}$and 0 to be the puncture. That the end and puncture of $\mathbf{S}(0)_{+}$are isomorphism invariants follows from [12], Proposition 2.5: indeed, any automorphism of $\mathbf{S}(0)_{+}$restricts to the identity map on its skeleton $\sigma((0, \infty))$, hence takes $0=\lim _{r \rightarrow \infty} \sigma(r)$ to 0 and $x=\lim _{r \rightarrow 0} \sigma(r)$ to $x$. Therefore, it makes sense to speak of the end and the puncture of any punctured open ball. If $A$ is a punctured open ball with end $x$ and puncture $y$, then we let $\bar{A}$ denote the compact space $A \cup\{x, y\}$.

Let $A$ be a generalized open annulus. Each connected component of $A \backslash \Sigma(A)$ is an open ball ([12], Lemma 2.12), and if $B$ is such a connected component with end $x$, then the inclusion $B \hookrightarrow A$ extends to an inclusion $\bar{B} \hookrightarrow A$ with $x$ mapping into $\Sigma(A)$; the image of $\bar{B}$ is the closure of $B$ in $A$. We define the retraction to the skeleton $\tau: A \rightarrow \Sigma(A)$ by fixing $\Sigma(A)$ and sending each connected component of $A \backslash \Sigma(A)$ to its end. If $A=\mathbf{S}(a)_{+}$, then the retraction $\tau$ coincides with $\sigma \circ$ val : $\mathbf{S}(a)_{+} \rightarrow \Sigma\left(\mathbf{S}(a)_{+}\right)$; in particular, if $(r, s) \subset$ $(0, \operatorname{val}(a))=\mathbf{S}(a)_{+}$, then $\tau^{-1}((r, s))=\operatorname{val}^{-1}((r, s))$.

\subsection{The skeleton of a curve}

Let $X$ be a smooth, connected, proper algebraic $K$-curve, and let $D \subset X(K)$ be a finite set of closed points. The set $\mathbf{H}\left(X^{\mathrm{an}}\right)$ is natually a metric space ([12], Corollary 5.7), although the metric topology on $\mathbf{H}\left(X^{\mathrm{an}}\right)$ is much finer than the topology induced by the topology on the $K$-analytic space $X^{\text {an }}$. The metric on $\mathbf{H}\left(X^{\text {an }}\right)$ is locally modeled on an $\mathbf{R}$-tree ([12], Proposition 5.9).

Definition 3.8. A semistable vertex set of $X$ is a finite set $V$ of type-2 points of $X^{\text {an }}$ such that $X^{\text {an }} \backslash V$ is a disjoint union of open balls and finitely many open annuli. A semistable vertex set of $(X, D)$ is a semistable vertex set of $X$ such that the points of $D$ are contained in distinct open ball connected components of $X^{\text {an }} \backslash V$.

It is a consequence of the semistable reduction theorem of Deligne-Mumford that there exist semistable vertex sets of $(X, D)$ : see ([12], Theorem 4.22). In the sequel, it will be convenient to consider a curve along with a choice of semistable vertex set, so we give such an object a name. 
Definition 3.9. A triangulated punctured curve $(X, V \cup D)$ is a smooth, connected, proper algebraic $K$-curve $X$ equipped with a finite set $D \subset X(K)$ of punctures and a semistable vertex set $V$ of $(X, D)$.

Remark 3.10. This terminology is loosely based on that used in [26] as well as the forthcoming book of Ducros on analytic spaces and analytic curves. Strictly speaking, what we have defined should be called a semistably triangulated punctured curve, but as these are the only triangulations that we consider, we will not need the added precision.

Let $(X, V \cup D)$ be a triangulated punctured curve, so

$$
X^{\text {an }} \backslash(V \cup D)=A_{1} \cup \cdots \cup A_{n} \cup \bigcup_{\alpha} B_{\alpha}
$$

where each $A_{i}$ is a generalized open annulus and $\left\{B_{\alpha}\right\}$ is an infinite collection of open balls. The skeleton of $(X, V \cup D)$ is the subset

$$
\Sigma(X, V \cup D)=V \cup D \cup \Sigma\left(A_{1}\right) \cup \cdots \cup \Sigma\left(A_{n}\right) .
$$

(This set is denoted $\widehat{\Sigma}(X, V \cup D)$ in [12].) For each $i$ and each $\alpha$ the inclusions $\mathbf{H}\left(A_{i}\right) \hookrightarrow$ $\mathbf{H}\left(X^{\text {an }}\right)$ and $\mathbf{H}\left(B_{\alpha}\right) \hookrightarrow \mathbf{H}\left(X^{\text {an }}\right)$ are local isometries ([12], Proposition 5.5). For each open ball $B_{\alpha}$, the map $B_{\alpha} \hookrightarrow X^{\text {an }}$ extends to an inclusion $\bar{B}_{\alpha} \hookrightarrow X^{\text {an }}$ sending the end of $B_{\alpha}$ to a point $x_{\alpha} \in V$. We say that $B_{\alpha}$ is adjacent to $x_{\alpha}$. For each open annulus $A_{i}$, the map $A_{i} \hookrightarrow X^{\text {an }}$ extends to a continuous map $\bar{A}_{i} \hookrightarrow X^{\text {an }}$ sending the ends of $A_{i}$ to points $x_{i}, y_{i} \in V$. We say that $A_{i}$ is adjacent to $x_{i}$ and $y_{i}$. The length $\ell\left(e_{i}\right) \in \Lambda$ of the geodesic segment $e_{i}=\Sigma\left(A_{i}\right) \cup\left\{x_{i}, y_{i}\right\}$ is then the modulus of $A_{i}$. We say that $V$ is strongly semistable if $x_{i} \neq y_{i}$ for each open annulus $A_{i}$. For each generalized open annulus $A_{i}$, the map $A_{i} \hookrightarrow$ $X^{\text {an }}$ extends to a continuous map $\bar{A}_{i} \hookrightarrow X^{\text {an }}$ sending the end of $A_{i}$ to a point $x_{i} \in V$ and sending the puncture to a point $y_{i} \in D$. We say that $A_{i}$ is adjacent to $x_{i}$ and $y_{i}$. We define the length of $e_{i}=\Sigma\left(A_{i}\right) \cup\left\{x_{i}, y_{i}\right\}$ to be $\ell\left(e_{i}\right)=\infty$.

The skeleton $\Sigma=\Sigma(X, V \cup D)$ naturally has the structure of a $\Lambda$-metric graph with distinguished finite vertex set $V_{f}(\Sigma)=V$, infinite vertex set $V_{\infty}(\Sigma)=D$, and edges $\left\{e_{1}, \ldots, e_{n}\right\}$ as above. Note that the $\Lambda$-points of $\Sigma$ are exactly the type-2 points of $X^{\text {an }}$ contained in $\Sigma$. For $x \in V$, the residue field $\widetilde{\mathscr{H}}(x)$ of the completed residue field $\mathscr{H}(x)$ at the type-2 point $x$ is a finitely generated field extension of $k$ of transcendence degree 1 ; we let $C_{x}$ be the smooth $k$-curve with function field $\widetilde{\mathscr{H}}(x)$. For $x \in V$ we let $g(x)$ be the genus of $C_{x}$, and for $x \in \Sigma \backslash V$ we set $g(x)=0$. These extra data give $\Sigma$ the structure of an augmented $\Lambda$-metric graph. (In (3.22), we will see that $\Sigma$ is in fact naturally a metrized complex of $k$-curves.) By the genus formula ([12], (4.1)), the genus of $X$ is equal to the genus of the augmented $\Lambda$-metric graph $\Sigma$.

The open analytic domain $X^{\text {an }} \backslash \Sigma$ is isomorphic to an infinite disjoint union of open balls ([12], Lemma 3.4(3)). If $B$ is a connected component of $X^{\text {an }} \backslash \Sigma$, then the inclusion $B \hookrightarrow X^{\text {an }}$ extends to a map $\bar{B} \hookrightarrow X^{\text {an }}$ sending the end of $B$ to a point of $\Sigma$. We define the retraction $\tau=\tau_{\Sigma}: X^{\text {an }} \rightarrow \Sigma$ by fixing $\Sigma$ and sending a point $x \in X^{\text {an }}$ not in $\Sigma$ to the end of the connected component of $x$ in $X^{\text {an }} \backslash \Sigma$. This is a continuous map and in fact Berkovich has proved that it is a strong deformation retraction, cf. [14]. If $x \in B_{\alpha}$ is in an open ball connected component of $X^{\text {an }} \backslash(V \cup D)$, then $\tau(x) \in V$ is the end of $B_{\alpha}$, and if $x \in A_{i}$, then $\tau(x)$ coincides with the image of $x$ under the retraction map $\tau: A_{i} \rightarrow \Sigma\left(A_{i}\right)$. 
Here, we collect some additional facts about skeleta from [12].

Proposition 3.12. Let $(X, V \cup D)$ be a triangulated punctured curve with skeleton $\Sigma=$ $\Sigma(X, V \cup D)$.

1. The skeleton $\Sigma$ is the set of points in $X^{\text {an }}$ that do not admit an open neighborhood isomorphic to $\mathbf{B}(1)_{+}$and disjoint from $V \cup D$.

2. Let $V_{1}$ be a semistable vertex set of $(X, D)$ such that $V_{1} \supset V$. Then, $\Sigma\left(X, V_{1} \cup D\right) \supset \Sigma(X, V \cup D)$ and $\tau_{\Sigma(X, V \cup D)}=\tau_{\Sigma(X, V \cup D)} \circ \tau_{\Sigma\left(X, V_{1} \cup D\right)}$.

3. Let $W \subset X^{\text {an }}$ be a finite set of type-2 points. Then, there exists a semistable vertex set of $(X, D)$ containing $V \cup W$.

4. Let $W \subset \Sigma$ be a finite set of type-2 points. Then, $V \cup W$ is a semistable vertex set of $(X, D)$ and $\Sigma(X, V \cup D)=\Sigma(X, V \cup W \cup D)$.

5. Let $x, y \in \Sigma \cap \mathbf{H}\left(X^{\text {an }}\right)$. Then, any geodesic segment from $x$ to $y$ in $\mathbf{H}\left(X^{\text {an }}\right)$ is contained in $\Sigma$.

6. Let $x, y \in X^{\text {an }}$ be points of type 2 or 3 , and let $[x, y]$ be a geodesic segment from $x$ to $y$ in $\mathbf{H}\left(X^{\text {an }}\right)$. Then, there exists a semistable vertex set $V_{1}$ of $(X, D)$ such that $V_{1} \supset V$ and $[x, y] \subset \Sigma\left(X, V_{1} \cup D\right)$.

Definition 3.13. A skeleton of $(X, D)$ is a subset of $X^{\text {an }}$ of the form $\Sigma=\Sigma(X, V \cup D)$ for some semistable vertex set $V$ of $(X, D)$. Such a semistable vertex set $V$ is called a vertex set for $\Sigma$. A skeleton of $X$ is a skeleton of $(X, \emptyset)$.

The augmented $\Lambda$-metric graph structure of a skeleton $\Sigma$ of $(X, D)$ does not depend on the choice of vertex set for $\Sigma$.

\subsection{Modifying the skeleton}

Let $X$ be a smooth, proper, connected $K$-curve, and let $D \subset X(K)$ be a finite set. Let $\Sigma$ be a skeleton of $(X, D)$, let $y \in X^{\text {an }} \backslash \Sigma$, let $B$ be the connected component of $X^{\text {an }} \backslash \Sigma$ containing $y$, and let $x=\tau(y) \in \Sigma$ be the end of $B$. If $y \in \mathbf{H}\left(X^{\text {an }}\right)$, then the geodesic segment $[x, y] \subset \bar{B}$ is the unique geodesic segment in $\mathbf{H}\left(X^{\text {an }}\right)$ connecting $x$ and $y$. If $y \in X(K)$, then we define $[x, y]$ to be the geodesic segment $[x, y] \subset \bar{B}$ as in (3.1). The following strengthening of Proposition 3.12(3) will be important in the sequel.

Lemma 3.15. Let $V$ be a semistable vertex set of $(X, D)$, let $\Sigma=\Sigma(X, V \cup D)$, let $W \subset X^{\text {an }}$ be a finite set of type-2 points, and let $E \subset X(K)$ be a finite set of type-1 points.

1. There exists a minimal semistable vertex set $V_{1}$ of $(X, D \cup E)$ which contains $V \cup W$, in the sense that any other such semistable vertex set contains $V_{1}$.

2. Let $B$ be a connected component of $X^{\text {an }} \backslash \Sigma$ with end $x$, and let $y_{1}, \ldots, y_{n}$ be the points of $(W \cup E) \cap B$. Then

$$
\Sigma\left(X, V_{1} \cup D \cup E\right) \cap \bar{B}=\left[x, y_{1}\right] \cup \cdots \cup\left[x, y_{n}\right]
$$

if $n>0$, and $\Sigma\left(X, V_{1} \cup D \cup E\right) \cap \bar{B}=\{x\}$ otherwise. Therefore,

$$
\Sigma\left(X, V_{1} \cup D \cup E\right)=\Sigma \cup \bigcup_{y \in W \cup E}\left[\tau_{\Sigma}(y), y\right]
$$


3. The skeleton $\Sigma_{1}=\Sigma\left(X, V_{1} \cup D \cup E\right)$ is minimal in the sense that any other skeleton containing $\Sigma$ and $W \cup E$ must contain $\Sigma_{1}$.

Proof. To prove the first part, we may assume that $W=\{y\}$ consists of a single type-2 point not contained in $\Sigma$ and $E=\emptyset$, or $E=\{y\}$ is a single type-1 point not contained in $D$ and $W=\emptyset$. In the first case, one sees using Lemma 3.4 that $V_{1}=V \cup\{y, \tau(y)\}$ is the minimal semistable vertex set of $(X, D)$ containing $V$ and $y$, and in the second case, $V_{1}=V \cup\{\tau(y)\}$ is the minimal semistable vertex set of $(X, D \cup\{y\})$ containing $V$. For $W$ and $E$ arbitrary, let $V_{1}$ be the minimal semistable vertex set of $(X, D \cup E)$ containing $V \cup W$ and let $\Sigma_{1}=\Sigma\left(X, V_{1} \cup D\right)$. If $E=\emptyset$, then it is clear from Proposition 3.12(5) that $\left[x, y_{i}\right] \subset \Sigma_{1}$ for each $i$; the other inclusion is proved by induction on $n$, adding one point at a time as above. The case $E \neq \emptyset$ is similar and is left to the reader. The final assertion follows easily from the first two and Proposition 3.12.

Remark 3.16. The above lemma shows in particular that the metric graph $\Sigma\left(X, V_{1} \cup\right.$ $D \cup E)$ is obtained from $\Sigma(X, V \cup D)$ by a sequence of tropical modifications and their inverses (see Definition II.2.12 and Example II.2.16). It is easy to see that any tropical modification of $\Sigma(X, V \cup D)$ is of the form $\Sigma\left(X, V_{1} \cup D \cup E\right)$ for a semistable vertex set $V_{1}$ which contains $V$ and a finite subset $E \subset X(K)$.

\subsection{The minimal skeleton}

The Euler characteristic of $X \backslash D$ is defined to be $\chi(X \backslash D)=2-2 g(X)-\# D$, where $g(X)$ is the genus of $X$. We say that $(X, D)$ is stable if $\chi(X \backslash D)<0$. A semistable vertex set $V$ of $(X, D)$ is minimal if there is no semistable vertex set of $(X, D)$ properly contained in $V$, and $V$ is stable if there is no $x \in V$ of genus zero (resp. one) and valency less than 3 (resp. one) in $\Sigma(X, V \cup D)$. It is clear that minimal semistable vertex sets exist. A skeleton $\Sigma$ of $(X, D)$ is minimal if there is no skeleton of $(X, D)$ properly contained in $\Sigma$.

The following consequence of the stable reduction theorem can be found in [12], Theorem 4.22 .

Proposition 3.18. Let $V$ be a minimal semistable vertex set of $(X, D)$, and let $\Sigma=$ $\Sigma(X, V \cup D)$.

1. If $\chi(X \backslash D) \leq 0$, then $\Sigma$ is the unique minimal skeleton of $(X, D)$; moreover, $\Sigma$ is equal to the set of points of $X^{\text {an }} \backslash D$ that do not admit an open neighborhood isomorphic to $\mathbf{B}(1)_{+}$and disjoint from $D$.

2. If $\chi(X \backslash D)<0$, then $V$ is the unique minimal semistable vertex set of $(X, D), V$ is stable, and

$$
V=\{x \in \Sigma: x \text { has valency } \geq 3 \text { or genus } \geq 1\} .
$$

Remark 3.19. We have $\chi(X \backslash D)>0$ if and only if $X \cong \mathbf{P}^{1}$ and $D$ contains at most one point; in this case, any type-2 point of $X^{\text {an }}$ serves as a minimal semistable vertex set of $(X, D)$, and there does not exist a unique minimal skeleton. If $\chi(X \backslash D)=0$, then either $X \cong \mathbf{P}^{1}$ and $D$ consists of two points, or $X$ is an elliptic curve and $D$ is empty. In the first case, the minimal skeleton $\Sigma$ of $(X, D)$ is the extended line connecting the points of $D$ and any type-2 point on $\Sigma$ is a minimal semistable vertex set. If $X$ is an elliptic curve and 
$X^{\text {an }}$ contains a type- 2 point $x$ of genus 1 , then $\{x\}$ is both the unique minimal semistable vertex set and the minimal skeleton. Otherwise, $X$ is a Tate curve, $\Sigma$ is a circle, and any type-2 point of $\Sigma$ is a minimal semistable vertex set. See [12], Remark 4.24.

\subsection{Tangent directions and the slope formula}

As above, we let $X$ be a smooth, proper, connected algebraic $K$-curve. A continuous function $F: X^{\text {an }} \rightarrow \mathbf{R} \cup\{ \pm \infty\}$ is called piecewise affine provided that $F\left(\mathbf{H}\left(X^{\mathrm{an}}\right)\right) \subset \mathbf{R}$ and $F \circ \alpha:[a, b] \rightarrow \mathbf{R}$ is a piecewise affine function for every geodesic segment $\alpha:[a, b] \hookrightarrow$ $\mathbf{H}\left(X^{\text {an }}\right)$. To any point, $x \in X^{\text {an }}$ is associated a set $T_{x}$ of tangent directions at $x$, defined as the set of germs of geodesic segments in $X^{\text {an }}$ beginning at $x$. If $F: X^{\text {an }} \rightarrow \mathbf{R} \cup\{ \pm \infty\}$ is a piecewise affine function and $v \in T_{x}$, we denote by $d_{v} F(x)$ the outgoing slope of $F$ in the direction $v$. We say that $F$ is harmonic at a point $x \in X^{\text {an }}$ provided that there are only finitely many $v \in T_{x}$ with $d_{v} F(x) \neq 0$, and $\sum_{v \in T_{x}} d_{v} F(x)=0$. See [12], (5.11).

Let $x \in X^{\text {an }}$ be a type- 2 point, let $\mathscr{H}(x)$ be the completed residue field of $X^{\text {an }}$ at $x$, and let $\widetilde{\mathscr{H}}(x)$ be its residue field, as in (3.11.1). Then, $\widetilde{\mathscr{H}}(x)$ is a finitely generated field extension of $k$ of transcendence degree 1 , and there is a canonical bijection between the set $T_{x}$ of tangent directions to $X^{\text {an }}$ at $x$ and the set $\mathrm{DV}(\widetilde{\mathscr{H}}(x) / k)$ of discrete valuations on $\widetilde{\mathscr{H}}(x)$ which are trivial on $k$. For $v \in T_{x}$, we let $\operatorname{ord}_{v}: \widetilde{\mathscr{H}}(x) \rightarrow \mathbf{Z}$ denote the corresponding valuation. Let $f$ be a nonzero analytic function on $X^{\text {an }}$ defined on a neighborhood of $x$, let $c \in K^{\times}$be such that $|c|=|f(x)|$, and let $\widetilde{f}_{x} \in \widetilde{\mathscr{H}}(x)$ denote the residue of $c^{-1} f$. Then, $\widetilde{f}_{x}$ is defined up to multiplication by $k^{\times}$, so for any $v \in T_{x}$, the integer $\operatorname{ord}_{v}\left(\tilde{f}_{x}\right)$ is well-defined.

The following theorem is called the slope formula in [12], Theorem 5.15:

Theorem 3.21. Let $f \in K(X)^{\times}$be a nonzero rational function on $X$ and let $F=-\log |f|$ : $X^{a n} \rightarrow \mathbf{R} \cup\{ \pm \infty\}$. Let $D \subset X(K)$ contain the zeros and poles off and let $\Sigma$ be a skeleton of $(X, D)$. Then:

1. $F=F \circ \tau$, where $\tau: X^{\text {an }} \rightarrow \Sigma$ is the retraction.

2. $F$ is piecewise affine with integer slopes, and $F$ is affine on each edge of $\Sigma$ (with respect to a choice of vertex set $V$ ).

3. If $x$ is a type-2 point of $X^{\text {an }}$ and $v \in T_{x}$, then $d_{v} F(x)=\operatorname{ord}_{v}\left(\tilde{f}_{x}\right)$.

4. $\quad F$ is harmonic at all points of $X^{\text {an }} \backslash D$.

5. Let $x \in X(K)$, and let $v$ be the unique tangent direction at $x$. Then, $d_{\nu} F(x)=\operatorname{ord}_{x}(f)$.

\subsection{The skeleton as a metrized complex of curves}

Let $(X, V \cup D)$ be a triangulated punctured curve with skeleton $\Sigma=\Sigma(X, V \cup D)$. Recall that $\Sigma$ is an augmented $\Lambda$-metric graph with infinite vertices $D$. We enrich $\Sigma$ with the structure of a $\Lambda$-metrized complex of $k$-curves as follows. For $x \in V$, let $C_{x}$ be the smooth, proper, connected $k$-curve with function field $\widetilde{\mathscr{H}}(x)$ as in (3.11.1). By definition, $C_{x}$ has genus $g(x)$. We have natural bijections

$$
T_{x} \cong \mathrm{DV}(\widetilde{\mathscr{H}}(x) / k) \cong C_{x}(k)
$$

where the first bijection $v \mapsto \operatorname{ord}_{v}$ is defined in (3.20) and the second associates to a closed point $\xi \in C_{x}(k)$ the discrete valuation ord $\xi$ on the function field $\widetilde{\mathscr{H}}(x)$ of $C_{x}$. Let $v \in T_{x}$ be a tangent direction and define $\operatorname{red}_{x}(v)$ to be the point of $C_{x}$ corresponding to the 
discrete valuation $\operatorname{ord}_{v} \in \mathrm{DV}(\widetilde{\mathscr{H}}(x) / k)$. These data make $\Sigma$ into a $\Lambda$-metrized complex of $k$-curves.

\subsection{Lifting metrized complexes of curves}

We now prove that every metrized complex of curves over $k$ arises as the skeleton of a smooth, proper, connected $K$-curve. This fact appears in the literature in various contexts (over discretely valued fields): see for instance [6], Appendix B, and [41], Lemme 6.3. For this reason, we only sketch a proof using our methods.

Theorem 3.24. Let $\mathcal{C}$ be a $\Lambda$-metrized complex of $k$-curves. There exists a triangulated punctured curve $(X, V \cup D)$ such that the skeleton $\Sigma(X, V \cup D)$ is isomorphic to $\mathcal{C}$.

Proof. Let $C$ be a smooth, proper, connected $k$-curve. By elementary deformation theory, there is a smooth, proper admissible formal $R$ scheme $\mathfrak{C}$ with special fiber $C$. By GAGA, the analytic generic fiber $\mathfrak{C}_{K}$ is the analytification of a smooth, proper, connected $K$-curve $\mathscr{C}$ (in fact, the formal scheme $\mathfrak{C}$ is itself algebraizable, see e.g. [25], Théorème 3.7.6). There is a reduction map red : $\mathfrak{C}_{K} \rightarrow C$ from the analytic generic fiber of $\mathfrak{C}$ to (the set underlying) $C$, under which the inverse image of the generic point of $C$ is a single distinguished point $x$. The set $\{x\}$ is a semistable vertex set of $\mathscr{C}$, with associated skeleton also equal to $\{x\}$. Moreover, there is a canonical identification of $\widetilde{\mathscr{H}}(x)$ with the field of rational functions on $\bar{C}$ by [14], Proposition 2.4.4. See (5.6) for a more detailed discussion of the relationship between semistable vertex sets and admissible formal models.

Let $\Gamma$ be the metric graph underlying $\mathcal{C}$. Let $V$ be the vertices of $\Gamma$, and let $E$ be its edges. By adding valence- 2 vertices, we may assume that $\Gamma$ has no loop edges. Assume for the moment that $\Gamma$ has no infinite edges. For a vertex $x \in V$, let $C_{x}$ denote the smooth, proper, connected $k$-curve associated to $x$ and choose an admissible formal curve $\mathfrak{C}_{x}$ with special fiber isomorphic to $C_{x}$ as above. For clarity, we let red $\mathfrak{C}_{x}$ denote the reduction map $\left(\mathfrak{C}_{x}\right)_{K} \rightarrow C_{x}$. By [19], Proposition 2.2, for every $\bar{x} \in C_{x}(k)$, the formal fiber $\operatorname{red}_{\mathfrak{C}_{x}}^{-1}(\bar{x})$ is isomorphic to $\mathbf{B}(1)_{+}$. Let $e$ be an edge of $\Gamma$ with endpoints $x, y$, and let $\bar{x}_{e} \in C_{x}(k), \bar{y}_{e} \in$ $C_{y}(k)$ be the reductions of the tangent vectors in the direction of $e$ at $x, y$, respectively. Remove closed balls from $\operatorname{red}_{\mathfrak{C}_{x}}^{-1}\left(\bar{x}_{e}\right)$ and $\operatorname{red}_{\mathfrak{C}_{y}}^{-1}\left(\bar{y}_{e}\right)$ whose radii are such that the remaining open annuli have modulus equal to $\ell(e)$. We form a new analytic curve $X^{\text {an }}$ by gluing these annuli together using some isomorphism of annuli for each edge $e$. The resulting curve is proper, hence is the analytification of an algebraic curve $X$. By construction, the image of the set of distinguished points of the curves $\left(\mathfrak{C}_{x}\right)_{K}$ in $X^{\text {an }}$ is a semistable vertex set, and the resulting skeleton (considered as a metric graph) is isomorphic to $\Gamma$.

If $\Gamma$ does have infinite edges, then we apply the above procedure to the finite part of $\Gamma$, then puncture the curve $X$ in the formal fibers over the smooth points of the residue curves which correspond to the directions of the infinite tails of $\Gamma$.

As an immediate application, we obtain the following property of the 'abstract tropicalization map' from the moduli space of stable marked curves to the moduli space of stable abstract tropical curves:

Corollary 3.25. If $g$ and $n$ are nonnegative integers with $2-2 g-n<0$, the natural map trop : $M_{g, n} \rightarrow M_{g, n}^{\text {trop }}$ (see [12], Remark 4.25) is surjective. 
See [1] for an interpretation of the above map as a contraction from $M_{g, n}^{\mathrm{an}}$ onto its skeleton (which also implies surjectivity).

\section{Morphisms between curves and their skeleta}

This section is a relative version of the previous one, in that we propose to study the behavior of semistable vertex sets and skeleta under finite morphisms of curves. We introduce finite morphisms of triangulated punctured curves, and we prove that any finite morphism of punctured curves can be enriched to a finite morphism of triangulated punctured curves. This powerful result can be used to prove the simultaneous semistable reduction theorems of Coleman, Liu-Lorenzini, and Liu, which we do in Section 5. It is interesting to note that we use only analytic methods on analytic $K$-curves, making (almost) no explicit reference to semistable models; hence, our proofs of the results of Liu-Lorenzini and Liu are very different from theirs. Using a relative version of the slope formula, we also show that a finite morphism of triangulated punctured curves induces (by restricting to skeleta) a finite harmonic morphism of metrized complexes of curves, which will be crucial in the sequel.

\subsection{Morphisms between open balls and generalized open annuli}

The main results of this section rest on a careful study of the behavior of certain morphisms between open balls and generalized open annuli. Some of the lemmas and intermediate results appear in some form in the literature - see in particular [14] and [15] - although they are hard to find in the form we need them. As the proofs are not difficult, for the convenience of the reader we include complete arguments.

We begin by remarking that if $\varphi: X^{\prime} \rightarrow X$ is a morphism of smooth strictly $K$-analytic curves with finite fibers, then $\varphi$ preserves the type of a point, i.e. if $x^{\prime} \in X^{\prime}$ has type $i$ then $x=\varphi\left(x^{\prime}\right)$ has type $i$. This is because $\varphi^{-1}(x)$ is a strictly $\mathscr{H}(x)$-analytic space of dimension zero, so $\left[\mathscr{H}\left(x^{\prime}\right): \mathscr{H}(x)\right]<\infty$.

Lemma 4.2. Let $a, a^{\prime} \in \mathfrak{m}_{R}$, and let $\varphi: \mathbf{S}\left(a^{\prime}\right)_{+} \rightarrow \mathbf{S}(a)_{+}$be a morphism.

1. If valo $\varphi$ is constant on $\Sigma\left(\mathbf{S}\left(a^{\prime}\right)_{+}\right)$, then $\varphi\left(\mathbf{S}\left(a^{\prime}\right)_{+}\right)$is contained in $\mathbf{S}(a)_{+} \backslash \Sigma\left(\mathbf{S}(a)_{+}\right)$.

2. If val $\circ \varphi$ is not constant on $\Sigma\left(\mathbf{S}\left(a^{\prime}\right)_{+}\right)$, then $\varphi\left(\Sigma\left(\mathbf{S}\left(a^{\prime}\right)_{+}\right)\right) \subset \Sigma(\mathbf{S}(a))$ and the restriction of $\varphi$ to $\Sigma\left(\mathbf{S}\left(a^{\prime}\right)_{+}\right)$has the form $x \mapsto m \cdot x+b$ for some nonzero integer $m$ and some $b \in \Lambda$.

3. If $\varphi$ extends to a continuous map $\left.\left.\bar{\varphi}: \mathbf{S} \overline{\left(a^{\prime}\right.}\right)_{+} \rightarrow \mathbf{S} \overline{(a}\right)_{+}$taking an end of $\mathbf{S}\left(a^{\prime}\right)_{+}$to an end of $\mathbf{S}(a)_{+}$, then val $\circ \varphi$ is not constant on $\Sigma\left(\mathbf{S}\left(a^{\prime}\right)_{+}\right)$.

Proof. Part (2) is exactly [12], Proposition 2.5, so suppose that val $\circ \varphi$ is constant on $\Sigma(\mathbf{S}(a))$. The map $\varphi: \mathbf{S}\left(a^{\prime}\right)_{+} \rightarrow \mathbf{S}(a)_{+} \subset \mathbf{G}_{m}^{\text {an }}$ is given by a unit $f$ on $\mathbf{S}\left(a^{\prime}\right)_{+}$. By [12], Proposition 2.2, we can write $f=\alpha(1+g)$, where $\alpha \in K^{\times}$and $\left|g\left(x^{\prime}\right)\right|<1$ for all $x^{\prime} \in$ $\mathbf{S}\left(a^{\prime}\right)_{+}$. Hence, $\left|\varphi\left(x^{\prime}\right)-\alpha\right|<|\alpha|$ for all $x^{\prime} \in \mathbf{S}\left(a^{\prime}\right)_{+}$; here, $\left|\varphi\left(x^{\prime}\right)-\alpha\right|$ should be interpreted as the absolute value of the function $f-\alpha$ in $\mathscr{H}\left(x^{\prime}\right)$ or equivalently as the absolute value of the function $t-\alpha$ in $\mathscr{H}(\varphi(x))$, where $t$ is a parameter on $\mathbf{G}_{m}$. But $|t(x)-\alpha| \geq|\alpha|$ for every $x \in \Sigma(\mathbf{S}(a))$, so $\varphi\left(\mathbf{S}\left(a^{\prime}\right)_{+}\right) \cap \Sigma(\mathbf{S}(a))=\emptyset$.

Let $x^{\prime}$ be an end of $\mathbf{S}\left(a^{\prime}\right)_{+}$, let $x$ be an end of $\mathbf{S}(a)_{+}$, and suppose that $\bar{\varphi}\left(x^{\prime}\right)=x$. Since $x^{\prime}$ is a limit point of $\Sigma\left(\mathbf{S}\left(a^{\prime}\right)_{+}\right)$, there exists a sequence of points $x_{1}^{\prime}, x_{2}^{\prime}, \ldots \in \Sigma\left(\mathbf{S}\left(a^{\prime}\right)_{+}\right)$ 
converging to $x^{\prime}$. Then, $\varphi\left(x_{1}^{\prime}\right), \varphi\left(x_{2}^{\prime}\right), \ldots$ converge to $x$, so val $\left(\varphi\left(x_{1}^{\prime}\right)\right)$, val $\left(\varphi\left(x_{2}^{\prime}\right)\right), \ldots$ converges to $\operatorname{val}(x)$. Since each $\operatorname{val}\left(\varphi\left(x_{i}^{\prime}\right)\right) \in(0, \operatorname{val}(a))$ but $\operatorname{val}(x) \notin(0, \operatorname{val}(a))$, $\operatorname{val} \circ \varphi$ is not constant on $\Sigma\left(\mathbf{S}\left(a^{\prime}\right)_{+}\right)$.

Lemma 4.3. Let $B^{\prime}$ be an open ball, let $A$ be a generalized open annulus, and let $\varphi: B^{\prime} \rightarrow A$ be a morphism with finite fibers. Then, $\varphi\left(B^{\prime}\right) \cap \Sigma(A)=\emptyset$, and $\varphi$ extends to a continuous map $\bar{B}^{\prime} \rightarrow A$.

Proof. We identify $A$ with $\mathbf{S}(a)_{+}$for $a \in \mathfrak{m}_{R}$ and $B^{\prime}$ with $\mathbf{B}(1)_{+}$. Any unit on $B^{\prime}$ has constant absolute value, so val $\circ \varphi$ is constant on $B^{\prime}$. Since $\mathbf{B}(1)_{+} \backslash\{0\}$ is a generalized open annulus, and since the type- 1 point 0 maps to a type- 1 point of $A$, it follows from Lemma 4.2 that $\varphi\left(B^{\prime}\right) \cap \Sigma(A)=\emptyset$. Let $B$ be the connected component of $A \backslash \Sigma(A)$ containing $\varphi\left(B^{\prime}\right)$. Then, the morphism $B^{\prime} \rightarrow B \hookrightarrow A$ extends to a continuous map $\bar{B}^{\prime} \rightarrow \bar{B} \hookrightarrow A$.

Definition 4.4. Let $T$ be a metric space, and let $m \in \mathbf{R}_{>0}$. A continuous injection $\varphi:[a, b] \hookrightarrow T$ is an embedding with expansion factor $m$ provided that $r \mapsto \varphi(r / m)$ : $[m a, m b] \hookrightarrow T$ is a geodesic segment. A continuous injection $\varphi:[a, b] \hookrightarrow T$ is piecewise affine provided that there exist $a=a_{0}<a_{1}<\cdots<a_{r}=b$ and $m_{1}, \ldots, m_{r} \in \mathbf{R}_{>0}$ such that $\left.\varphi\right|_{\left[a_{i-1}, a_{i}\right]}$ is an embedding with expansion factor $m_{i}$ for each $i=1, \ldots, r$.

Lemma 4.5. Let $B, B^{\prime}$ be open balls, let $x^{\prime}$ be the end of $B^{\prime}$, and let $\varphi: B^{\prime} \rightarrow B$ be a morphism with finite fibers. Let $y^{\prime} \in B^{\prime}$, let $y=\varphi\left(y^{\prime}\right)$, and let $x=\varphi\left(x^{\prime}\right) \in \bar{B}^{\prime}$. Then, the restriction of $\varphi$ to the geodesic segment $\left[x^{\prime}, y^{\prime}\right]$ is injective, and $\varphi\left(\left[x^{\prime}, y^{\prime}\right]\right)$ is equal to the geodesic segment $[x, y]$. If in addition there exists $N>0$ such that all fibers of $\varphi$ have fewer than $N$ elements, then $\left.\varphi\right|_{\left[x^{\prime}, y^{\prime}\right]}$ is piecewise affine.

Proof. First suppose that $y^{\prime}$ does not have type 4 . Choose isomorphisms $B^{\prime} \cong \mathbf{B}(1)_{+}$ such that $0 \geq y^{\prime}$ and $B \cong \mathbf{B}(1)_{+}$such that $\varphi(0)=0$. Since $y^{\prime} \in\left[x^{\prime}, 0\right]$, we may replace $\left[x^{\prime}, y^{\prime}\right]$ by the larger geodesic segment $\left[x^{\prime}, 0\right]$ to assume that $y^{\prime}=0$. Define $F: \Sigma\left(\mathbf{S}(0)_{+}\right) \rightarrow$ $\mathbf{R}$ by $F(x)=$ val $\circ \varphi(x)=-\log |\varphi(x)|$. By [12], Proposition 2.10, $F$ is a piecewise affine function, and for any point $z^{\prime} \in \Sigma\left(\mathbf{S}(0)_{+}\right)$at which $F$ is differentiable, the derivative of $F$ is equal to the number of zeros $q^{\prime}$ of $\varphi$ with $\operatorname{val}\left(q^{\prime}\right)>\operatorname{val}\left(z^{\prime}\right)$. It follows that $F$ is monotonically increasing, so $\varphi$ is injective on $\left[x^{\prime}, 0\right]$. Let

$$
Z=\left\{\tau\left(q^{\prime}\right): q^{\prime} \in \mathbf{S}(0)_{+}, \varphi\left(q^{\prime}\right)=0\right\} \subset \Sigma\left(\mathbf{S}(0)_{+}\right) .
$$

If $C \subset \Sigma\left(\mathbf{S}(0)_{+}\right) \backslash Z$ is a connected component, then $A^{\prime}=\tau^{-1}(C)$ is a generalized open annulus mapping to $\mathbf{S}(0)_{+}$. By the above, val $\circ \varphi$ is not constant on $\Sigma\left(A^{\prime}\right)=\Sigma\left(\mathbf{S}(0)_{+}\right) \cap A^{\prime}$, so by Lemma 4.2, $\varphi\left(\Sigma\left(A^{\prime}\right)\right) \subset \Sigma\left(\mathbf{S}(0)_{+}\right)$. Since $\Sigma\left(\mathbf{S}(0)_{+}\right) \backslash Z$ is dense in $\Sigma\left(\mathbf{S}(0)_{+}\right)$, we have that $\left[x^{\prime}, 0\right]=\Sigma\left(\mathbf{S}(0)_{+}\right) \cup\{0\}$ maps bijectively onto $[x, 0]$. It is clear in this case that the restriction of $\varphi$ to the closure of any connected component of $\Sigma\left(\mathbf{S}(0)_{+}\right) \backslash Z$ is an embedding with integer expansion factor, so $\left.\varphi\right|_{\left[x^{\prime}, 0\right]}$ is piecewise affine. Note that $\left.\varphi\right|_{\left[x^{\prime}, 0\right]}$ changes expansion factor at most $\# \varphi^{-1}(0)$ times. 
Now suppose that $y^{\prime}$ has type 4 . Suppose that $z^{\prime}, w^{\prime} \in\left[x^{\prime}, y^{\prime}\right]$ are two distinct points such that $\varphi\left(z^{\prime}\right)=\varphi\left(w^{\prime}\right)$. Assume without loss of generality that $z^{\prime}<w^{\prime}$. Since $w^{\prime}$ and $z^{\prime}$ have the same image under $\varphi$, they both have the same type, so $w^{\prime} \neq y^{\prime}$ because $y^{\prime}$ is the only type- 4 point in $\left[x^{\prime}, y^{\prime}\right]$. Applying the above to the geodesic $\left[x^{\prime}, w^{\prime}\right]$ gives a contradiction. Therefore, $\varphi$ is injective on $\left[x^{\prime}, y^{\prime}\right]$, so $\varphi\left(\left[x^{\prime}, y^{\prime}\right]\right)=[x, y]$ since $B$ is uniquely path-connected ([9], Corollary 1.14).

Assume now that all fibers of $\varphi$ have size bounded by $N$. The above argument proves that for all $z^{\prime}<y^{\prime}$, the restriction of $\varphi$ to $\left[x^{\prime}, z^{\prime}\right]$ is piecewise affine, and $\left.\varphi\right|_{\left[x^{\prime}, z^{\prime}\right]}$ changes expansion factor at most $N$ times. Therefore, there exists $z_{0}^{\prime}<y^{\prime}$ and $m \in \mathbf{Z}_{>0}$ such that for all $z^{\prime} \in\left[z_{0}^{\prime}, y^{\prime}\right]$, the restriction of $\varphi$ to $\left[z_{0}^{\prime}, z^{\prime}\right]$ is an embedding with expansion factor $m$. It follows that $\left.\varphi\right|_{\left[z_{0}^{\prime}, y^{\prime}\right]}$ is an embedding with expansion factor $m$, so $\left.\varphi\right|_{\left[x^{\prime}, y^{\prime}\right]}$ is piecewise affine.

Lemma 4.6. Let $B, B^{\prime}$ be open balls, and let $\varphi: B^{\prime} \rightarrow B$ be a morphism. Suppose that $\varphi$ is open and has finite fibers, so it extends canonically to a continuous map $\varphi: \bar{B}^{\prime} \rightarrow \bar{B}$ by Lemma 3.3. Let $x^{\prime}$ be the end of $B^{\prime}$, and let $x=\varphi\left(x^{\prime}\right) \in \bar{B}$. Then, $B_{1}=\varphi\left(B^{\prime}\right)$ is an open ball connected component of $\bar{B} \backslash\{x\}$ and $\varphi: B^{\prime} \rightarrow B_{1}$ is finite and order-preserving. In particular, if $x$ is the end of $B$, then $\varphi: B^{\prime} \rightarrow B$ is finite and order-preserving.

Proof. Let $y^{\prime} \in B^{\prime}$. By Lemma 4.5, the restriction of $\varphi$ to the geodesic $\left[x^{\prime}, y^{\prime}\right]$ is injective, so $\varphi\left(y^{\prime}\right) \neq x$. Therefore $x \notin B_{1}$. Let $C$ be the connected component of $B \backslash\{x\}$ containing $B_{1}$. Since $x$ is an end of $C$ and $x^{\prime}$ maps to $x$, Lemma 4.3 implies that $C$ is not an open annulus, so it is an open ball. By hypothesis, $B_{1}$ is an open subset of $C$; since $B^{\prime} \cup\left\{x^{\prime}\right\}$ is compact, $\varphi\left(B^{\prime} \cup\left\{x^{\prime}\right\}\right)=B_{1} \cup\{x\}$ is closed in $\bar{B}$, and therefore $B_{1}=\left(B_{1} \cup\{x\}\right) \cap C$ is closed in $C$. Since $C$ is connected, we have $B_{1}=C$.

Since $\varphi$ has finite fibers, $\varphi: B^{\prime} \rightarrow B_{1}$ is finite if and only if it is proper by [14], Corollary 3.3.8. If $D \subset B_{1}$ is compact, then $\varphi^{-1}(D)$ is compact since $\varphi^{-1}(D)$ is closed as a subset of the compact space $\bar{B}^{\prime}$. One has $\operatorname{Int}\left(B^{\prime} / B_{1}\right)=B^{\prime}$ by [14], Proposition 3.1.3(i) since $B^{\prime}$ is a boundary-less $K$-analytic space. Therefore, $\varphi$ is proper.

The fact that $\varphi: B^{\prime} \rightarrow B_{1}$ is order-preserving follows immediately from Lemma 4.5.

Let $\varphi: B^{\prime} \rightarrow B$ be a finite morphism of open balls. Since an open ball is a smooth curve, $\varphi$ is flat in the sense that if $\mathscr{M}(\mathcal{A}) \subset B$ is an affinoid domain and $\varphi^{-1}(\mathscr{M}(\mathcal{A}))=\mathscr{M}\left(\mathcal{A}^{\prime}\right)$, then $\mathcal{A}^{\prime}$ is a (finite) flat $\mathcal{A}$-algebra. For $x \in \mathscr{M}(\mathcal{A})$, the fiber over $x$ is

$$
\varphi^{-1}(x)=\mathscr{M}\left(\mathcal{A}^{\prime} \widehat{\otimes}_{\mathcal{A}} \mathscr{H}(x)\right)=\mathscr{M}\left(\mathcal{A}^{\prime} \otimes_{\mathcal{A}} \mathscr{H}(x)\right),
$$

where the second equality holds because $\mathcal{A}^{\prime}$ is a finite $\mathcal{A}$-algebra. It follows that for $x \in B$ the quantity $\sum_{x^{\prime} \mapsto x} \operatorname{dim}_{\mathscr{H}(x)} \mathrm{O}_{\varphi^{-1}(x), x^{\prime}}$ is independent of $x$; we call this number the degree of $\varphi$.

Proposition 4.8. Let $B, B^{\prime}$ be open balls, and let $\varphi: B^{\prime} \rightarrow B$ be a finite morphism. Let $x^{\prime}$ be the end of $B^{\prime}$, let $x=\varphi\left(x^{\prime}\right)$ be the end of $B$, let $y \in B$, and let $y_{1}^{\prime}, \ldots, y_{n}^{\prime} \in B^{\prime}$ be the inverse images of $y$ in $B^{\prime}$. Then,

$$
\varphi^{-1}([x, y])=\left[x^{\prime}, y_{1}^{\prime}\right] \cup \cdots \cup\left[x^{\prime}, y_{n}^{\prime}\right]
$$


Proof. Let $T^{\prime}=\left[x^{\prime}, y_{1}^{\prime}\right] \cup \cdots \cup\left[x^{\prime}, y_{n}^{\prime}\right]$. The inclusion $T^{\prime} \subset \varphi^{-1}([x, y])$ follows from Lemma 4.5. First, we claim that for $z \in[x, y]$ near enough to $x$, there is only one preimage of $z$ in $B^{\prime}$. Shrinking $[x, y]$ if necessary, we may assume that $y$ has type 2 . Choose a type-1 point $w \in B$ such that $w>y$, so $(x, y] \subset(x, w)=\Sigma(B \backslash\{w\})$. Let $w^{\prime} \in B^{\prime}$ be a preimage of $w$ and choose $z^{\prime} \in \Sigma\left(B^{\prime} \backslash\left\{w^{\prime}\right\}\right)$ such that $z^{\prime} \leq \tau\left(q^{\prime}\right)$ for all $q^{\prime} \in \varphi^{-1}(w)$, where $\tau: B^{\prime} \backslash\left\{w^{\prime}\right\} \rightarrow \Sigma\left(B^{\prime} \backslash\left\{w^{\prime}\right\}\right)$ is the retraction. Let $A^{\prime}$ be the open annulus $\tau^{-1}\left(\left(x^{\prime}, z^{\prime}\right)\right) \subset B^{\prime}$. Then, $\varphi\left(A^{\prime}\right) \subset B \backslash\{w\}$, and $\varphi$ takes the end $x^{\prime}$ of $A^{\prime}$ to the end $x$ of $B \backslash\{w\}$, so by Lemma 4.2(2,3), $\varphi\left(\Sigma\left(A^{\prime}\right)\right) \subset \Sigma(B \backslash\{w\})$ and the map $\Sigma\left(A^{\prime}\right) \rightarrow$ $\Sigma(B \backslash\{w\})$ is injective. If $u^{\prime} \in A^{\prime} \backslash \Sigma\left(A^{\prime}\right)$, then $u^{\prime}$ is contained in an open ball in $A^{\prime}$, so $\varphi\left(u^{\prime}\right) \notin \Sigma(B \backslash\{w\})$ by Lemma 4.3. Therefore, every point of $\Sigma(B \backslash\{w\})$ has at most one preimage in $A^{\prime}$. Let $u^{\prime} \in B \backslash A^{\prime}$, so $u^{\prime} \geq z^{\prime}$. Then, $\varphi\left(u^{\prime}\right) \geq \varphi\left(z^{\prime}\right)$, so every point $u \in(x, y]$ with $u<\varphi\left(z^{\prime}\right)$ has exactly one preimage in $B^{\prime}$ (note that $\varphi\left(z^{\prime}\right) \in \Sigma(B \backslash\{w\}$ ) by Lemma 4.5).

Let $d$ be the degree of the finite morphism $\varphi$, so for every $z \in B$, we have

$$
d=\sum_{z^{\prime} \in \varphi^{-1}(z)} \operatorname{dim}_{\mathscr{H}(z)} \mathrm{O}_{\varphi^{-1}(z), z^{\prime}}
$$

For $y \in B$ as in the statement of the Proposition define a function $\delta:(x, y] \rightarrow \mathbf{Z}$ by

$$
\delta(z)=\sum_{\substack{z^{\prime} \in \varphi^{-1}(z) \\ z^{\prime} \in T^{\prime}}} \operatorname{dim} \mathscr{H}(z) \mathrm{O}_{\varphi^{-1}(z), z^{\prime}}
$$

Clearly, $\delta(z) \leq d$ for all $z \in(x, y]$, and $\delta(z)=d$ if and only if $\varphi^{-1}(z) \subset T^{\prime}$. By definition of $T^{\prime}$, we have $\delta(y)=d$, and by the above, $\delta(z)=d$ for $z \in(x, y]$ close enough to $x$ (any geodesic segment $\left[x^{\prime}, y_{i}^{\prime}\right]$ surjects onto $[x, y]$, hence contains the unique preimage of $z$ ). Therefore, it is enough to show that $\delta\left(z_{1}\right) \geq \delta\left(z_{2}\right)$ if $z_{1} \leq z_{2}$.

If $z \in B$ is a point of type 2 or 3 , then $B_{z}:=\{w \in B: w \geq z\}$ is an (not necessarily strict) affinoid subdomain of $B$. If $w^{\prime} \in \varphi^{-1}\left(B_{z}\right)$, i.e., if $\varphi\left(w^{\prime}\right) \geq z$, then by Lemma 4.5, $\varphi\left(\left[x^{\prime}, w^{\prime}\right]\right)=\left[x, \varphi\left(w^{\prime}\right)\right]$ is a geodesic containing $z$, so there exists $z^{\prime} \in\left[x^{\prime}, w^{\prime}\right]$ mapping to $z$. It follows that

$$
\varphi^{-1}\left(B_{z}\right)=\coprod_{z^{\prime} \mapsto z}\left\{w^{\prime} \in B^{\prime}: w^{\prime} \geq z^{\prime}\right\}=\coprod_{z^{\prime} \mapsto z} B_{z^{\prime}}^{\prime}
$$

is a disjoint union of affinoid domains: indeed, by Lemma 4.5, the preimages of $z$ are incomparable (otherwise, the restriction of $\varphi$ to a geodesic segment $\left[z^{\prime}, z^{\prime \prime}\right]$, for $z^{\prime}, z^{\prime \prime} \in$ $\varphi^{-1}(z)$, would not be injective), and so the sets $B_{z^{\prime}}$ are disjoint. Hence, each map $B_{z^{\prime}} \rightarrow B_{z}$ is finite, and its degree is equal to $\operatorname{dim}_{\mathscr{H}(z)} \mathrm{O}_{\varphi^{-1}(z), z^{\prime}}$. Let $z_{1}, z_{2} \in(x, y]$ and assume that $z_{1}<z_{2}$, so $z_{1}$ has type 2 or 3 . For $z_{1}^{\prime} \in \varphi^{-1}\left(z_{1}\right)$, we have

$$
\operatorname{dim}_{\mathscr{H}\left(z_{1}\right)} \mathrm{O}_{\varphi^{-1}\left(z_{1}\right), z_{1}^{\prime}}=\sum_{\substack{z_{2}^{\prime} \mapsto z_{2} \\ z_{2}^{\prime} \geq z_{1}^{\prime}}} \operatorname{dim} \mathscr{H}\left(z_{2}\right) \mathrm{O}_{\varphi^{-1}\left(z_{2}\right), z_{2}^{\prime}} .
$$


Summing over all $z_{1}^{\prime} \in \varphi^{-1}\left(z_{1}\right) \cap T^{\prime}$, we obtain

$$
\begin{aligned}
\delta\left(z_{1}\right) & \left.=\sum_{\substack{z_{1}^{\prime} \mapsto z_{1} \\
z_{1}^{\prime} \in T^{\prime}}} \operatorname{dim}_{\mathscr{H}\left(z_{1}\right)} \mathrm{O}_{\varphi^{-1}\left(z_{1}\right), z_{1}^{\prime}}=\sum_{\substack{z_{1}^{\prime} \mapsto z_{1} \\
z_{1}^{\prime} \in T^{\prime}}} \sum_{z_{2}^{\prime} \mapsto z_{2}} \operatorname{dim}_{z_{2}^{\prime} \geq z_{1}^{\prime}} \operatorname{He}_{2}\right) \mathrm{O}_{\varphi^{-1}\left(z_{2}\right), z_{2}^{\prime}} \\
& \geq \sum_{\substack{z_{1}^{\prime} \mapsto z_{1} \\
z_{1}^{\prime} \in T^{\prime}}} \sum_{\substack{z_{2}^{\prime} \mapsto z_{2} \\
z_{2}^{\prime} \geq z_{1}^{\prime} \\
z_{2}^{\prime} \in T^{\prime}}} \operatorname{dim}_{\mathscr{H}\left(z_{2}\right)} \mathrm{O}_{\varphi^{-1}\left(z_{2}\right), z_{2}^{\prime}}=\delta\left(z_{2}\right),
\end{aligned}
$$

where the final equality holds because if $z_{2}^{\prime} \in \varphi^{-1}\left(z_{2}\right) \cap T^{\prime}$, then there exists $z_{1}^{\prime} \in \varphi^{-1}\left(z_{1}\right) \cap$ $T^{\prime}$ such that $z_{2}^{\prime} \geq z_{1}^{\prime}$, namely, the unique point of $\left[x^{\prime}, z_{2}^{\prime}\right]$ mapping to $z_{1}$.

Proposition 4.9. Let $a \in \mathfrak{m}_{R} \backslash\{0\}$, let $A^{\prime}=\mathbf{S}(a)_{+}$, let $B$ be an open ball, and let $\varphi: A^{\prime} \rightarrow B$ be a morphism with finite fibers. Suppose that each end of $A^{\prime}$ maps to the end of $B$ or to a type-2 point of $B$ under the induced map $\bar{A}^{\prime} \rightarrow \bar{B}$. Let $\alpha=\varphi \circ \sigma:[0, \operatorname{val}(a)] \rightarrow \bar{B}$. Then, there exist finitely many numbers $r_{0}, r_{1}, r_{2}, \ldots, r_{n} \in \Lambda$ with $0=r_{0}<r_{1}<r_{2}<\cdots<r_{n}=$ $v a l(a)$ such that $\alpha$ is an embedding with nonzero integer expansion factor when restricted to each interval $\left[r_{i}, r_{i+1}\right]$. In other words, $\alpha$ is piecewise affine with integer expansion factors. Moreover, the image of $\alpha$ is a geodesic segment between type- 2 points of $B$.

Proof. Let $x^{\prime}$ be the end $\sigma(0)$ of $A^{\prime}$, and let $x=\varphi\left(x^{\prime}\right)$. Suppose first that $x$ is the end of $B$. Choose an identification $B \cong \mathbf{B}(1)_{+}$, and let $r_{+}=\min \left\{\operatorname{val}\left(y^{\prime}\right): \varphi\left(y^{\prime}\right)=0\right\}>0$ if the preimage of 0 is not empty; otherwise, set $r_{+}=\operatorname{val}(a)$. Let $A_{+}^{\prime}$ be the open annulus val $^{-1}\left(\left(0, r_{+}\right)\right) \subset A^{\prime}$. Then, $\varphi\left(A_{+}^{\prime}\right) \subset \mathbf{B}(1)_{+} \backslash\{0\}=\mathbf{S}(0)_{+}$and the end $x^{\prime}$ of $A_{+}^{\prime}$ maps to the end $x$ of $\mathbf{S}(0)_{+}$, so by Lemma 4.2(2,3), $\alpha$ is an embedding with (nonzero) integer expansion factor when restricted to $\left[0, r_{+}\right]$.

Now, suppose that $x$ is not the end of $B$. Let $r=\min \left\{\operatorname{val}(a),\left\{\operatorname{val}\left(y^{\prime}\right): \varphi\left(y^{\prime}\right)=x\right\}\right\} \in$ $(0, \operatorname{val}(a)]$, and let $A^{\prime \prime}=\mathrm{val}^{-1}((0, r))$. Then, $\varphi\left(A^{\prime \prime}\right)$ is contained in a connected component $C$ of $B \backslash\{x\}$, and $\varphi$ takes the end $x^{\prime}$ of $A^{\prime \prime}$ to the end $x$ of $C$. If $C$ is an open annulus, then $\alpha$ is an embedding with integer expansion factor when restricted to $[0, r]$ by Lemma 4.2(2,3); and if $C$ is an open ball, then we proceed as above to find $r_{+} \in(0, r] \cap \Lambda$ such that $\alpha$ is an embedding with integer expansion factor when restricted to $\left[0, r_{+}\right]$.

Applying the above argument to the morphism $\varphi$ composed with the automorphism $t \mapsto a / t$ of $\mathbf{S}(a)_{+}$(which interchanges the two ends), we find that there exists $r_{-} \in$ $[0, \operatorname{val}(a)) \cap \Lambda$ such that $\alpha$ is an embedding with integer expansion factor when restricted to $\left[r_{-}, \operatorname{val}(a)\right]$. Let $s \in(0, \operatorname{val}(a)) \cap \Lambda$. Replacing $A^{\prime}$ with the annulus val ${ }^{-1}((0, s))$ (resp. $\left.\operatorname{val}^{-1}((s, \operatorname{val}(a)))\right)$, the above arguments then provide us with $s_{+} \in(s, \operatorname{val}(a)] \cap \Lambda$ (resp. $\left.s_{-} \in[0, s) \cap \Lambda\right)$ such that $\alpha$ is an embedding with integer expansion factor when restricted to $\left[s, s_{+}\right]$(resp. $\left.\left[s_{-}, s\right]\right)$. Hence, we have shown that there exists a neighborhood $U_{s}$ of every point $s \in[0, \operatorname{val}(a)] \cap \Lambda$ such that $\left.\varphi\right|_{U_{s}}$ is an embedding with integer expansion factor.

It remains to treat type- 3 points, i.e., points $s \in[0, \operatorname{val}(a)] \backslash \Lambda$. Let $s$ be such a point, and let $y=\varphi(s)$. Then, $y$ has type 3 as well, so by [12], Corollary 4.27(2), there is an open neighborhood $U$ of $y$ which is isomorphic to an open annulus containing $y$ in its skeleton. For the same reason, $\varphi^{-1}(U)$ contains an open neighborhood $U^{\prime}$ of $s$ which is isomorphic 
to an open annulus containing $s$ in its skeleton. Again by Lemma 4.2(2,3), this implies that $\varphi$ is an embedding with integer expansion factor on $U_{s}:=U^{\prime} \cap[0, \operatorname{val}(a)]$.

The first assertions now follow because there is a finite subcover of the open covering $\left\{U_{s}: s \in[0, \operatorname{val}(a)]\right\}$ of the compact space $[0, \operatorname{val}(a)]$.

As for the final assertion, choose $0=r_{0}<r_{1}<r_{2}<\cdots<r_{n}=\operatorname{val}(a)$ such that $\alpha$ is an embedding with integer expansion factor on each $\left[r_{i}, r_{i+1}\right]$. Let $i_{0} \in\{0,1, \ldots, n\}$ be the largest integer such that $\alpha\left(r_{i_{0}}\right)>\alpha\left(r_{i}\right)$ (in the canonical partial ordering on $B$ ) for all $i<i_{0}$. If $i_{0}=n$, then we are done, so assume that $i_{0}<n$. Let $y=\alpha\left(r_{i_{0}}\right) \in B$, and choose an identification $B \cong \mathbf{B}(1)_{+}$such that $0>y$. If $F=-\log |\varphi|$, then by [12], Proposition 2.10, at every point $r \in(0, \operatorname{val}(a))$, the change in slope of $F$ at $r$ is equal to the negative of the number of zeros of $\varphi$ with valuation $r$ (cf. the proof of Lemma 4.5); in particular, the slope of $F$ can only decrease. By construction, $F$ is monotonically increasing on $\left[0, r_{i_{0}}\right]$. Since $\alpha\left(\left[r_{i_{0}}, r_{i_{0}+1}\right]\right)$ is a geodesic segment, it meets $y$ only at $\alpha\left(r_{i_{0}}\right)$. The image of $\left(r_{i_{0}}, r_{i_{0}+1}\right]$ under $\alpha$ is not contained in an open ball connected component of $B \backslash\{y\}$ because $\alpha\left(r_{i_{0}+1}\right) \ngtr y$; hence, $F$ is decreasing on an interval $\left[r_{i_{0}}, r_{i_{0}}+\epsilon\right]$ for some $\epsilon>0$. Since the slope of $F$ can only decrease, it follows that $F$ is monotonically decreasing on $\left[r_{i_{0}}, r_{n}\right]$. It follows immediately from this that $\alpha([0, \operatorname{val}(a)])=[x, y]$ or $\alpha([0, \operatorname{val}(a)])=[\alpha(\operatorname{val}(a)), y]$, whichever segment is larger.

\subsection{Morphisms between curves and skeleta}

In what follows, we fix smooth, connected, proper algebraic $K$-curves $X, X^{\prime}$ and a finite morphism $\varphi: X^{\prime} \rightarrow X$. Let $D \subset X(K)$ and $D^{\prime} \subset X^{\prime}(K)$ be finite sets of closed points. The map on analytifications $\varphi: X^{\prime a n} \rightarrow X^{\text {an }}$ is finite and open by [14], Lemma 3.2.4.

Proposition 4.11. Let $\Sigma^{\prime}$ be a skeleton of $\left(X^{\prime}, D^{\prime}\right)$, and let $\Sigma$ be a skeleton of $(X, D)$. There exists a skeleton $\Sigma_{1}$ of $\left(X, D \cup \varphi\left(D^{\prime}\right)\right)$ containing $\Sigma \cup \varphi\left(\Sigma^{\prime}\right)$, and there is a minimal such $\Sigma_{1}$ with respect to inclusion.

Proof. First, we will prove the Proposition in the case $D=D^{\prime}=\emptyset$. Let $V$ be a vertex set for $\Sigma$, and let $V^{\prime}$ be a vertex set for $\Sigma^{\prime}$ containing $\left\{\tau\left(y^{\prime}\right): y^{\prime} \in \varphi^{-1}\right\}$. Let $A^{\prime}$ be an open annulus connected component of $X^{\prime a n} \backslash V^{\prime}$, and let $e^{\prime} \subset \Sigma^{\prime}$ be the associated edge. We claim that $\varphi\left(e^{\prime}\right)$ is a geodesic segment between type-2 points of $X^{\text {an }}$. Let $C$ be the connected component of $X^{\text {an }} \backslash V$ containing $\varphi\left(A^{\prime}\right)$.

(i) If $C$ is an open ball, then the claim follows immediately from Proposition 4.9.

(ii) If $C$ is an open annulus and $\varphi\left(A^{\prime}\right) \cap \Sigma(C)=\emptyset$, then $A^{\prime}$ is contained in an open ball in $C$ because each connected component of $C \backslash \Sigma(C)$ is an open ball, so the claim is true as in (i).

(ii) If $C$ is an open annulus and $\varphi\left(A^{\prime}\right) \cap \Sigma(C) \neq \emptyset$, then $\varphi\left(e^{\prime}\right)$ is a geodesic segment with endpoints of type 2 by Lemma 4.2.

Applying the above to each edge $e^{\prime}$ of $\Sigma^{\prime}$, we find that there exists a finite set of type-2 points $x_{1}, y_{1}, x_{2}, y_{2}, \ldots, x_{n}, y_{n} \in X^{\text {an }}$ such that $\varphi\left(\Sigma^{\prime}\right)=\bigcup_{i=1}^{n}\left[x_{i}, y_{i}\right]$, where $\left[x_{i}, y_{i}\right]$ denotes a geodesic segment from $x_{i}$ to $y_{i}$. Let

$$
\Sigma_{1}=\Sigma \cup \bigcup_{i=1}^{n}\left(\left[x_{i}, \tau_{\Sigma}\left(x_{i}\right)\right] \cup\left[y_{i}, \tau_{\Sigma}\left(y_{i}\right)\right]\right) .
$$


By Lemma 3.15(2) as applied to $W=\left\{x_{1}, y_{1}, \ldots, x_{n}, y_{n}\right\}$, we have that $\Sigma_{1}$ is a skeleton of $X$, so by Proposition 3.12(5), $\Sigma_{1}$ contains $\Sigma \cup \varphi\left(\Sigma^{\prime}\right)$ since $\Sigma_{1}$ contains each geodesic segment $\left[x_{i}, y_{i}\right]$. Any other skeleton of $X$ containing $\Sigma$ and all $x_{i}, y_{i}$ also contains the geodesics $\left[x_{i}, \tau_{\Sigma}\left(x_{i}\right)\right]$ and $\left[y_{i}, \tau_{\Sigma}\left(y_{i}\right)\right]$, so $\Sigma_{1}$ is the minimal such skeleton.

Now, assume that $D \amalg D^{\prime} \neq \emptyset$. By Lemma 3.15(3), there is a minimal skeleton $\Sigma_{1}$ of $X$ containing $\Sigma$ and $\varphi\left(D^{\prime}\right)$; replacing $\Sigma$ by $\Sigma_{1}$ and $D$ by $D \cup \varphi\left(D^{\prime}\right)$, we may assume without loss of generality that $\varphi\left(D^{\prime}\right) \subset D$. We will proceed by induction on the size of $D \amalg D^{\prime}$. As above, we let $V$ be a vertex set for $\Sigma$, and we let $V^{\prime}$ be a vertex set for $\Sigma^{\prime}$ containing $\left\{\tau\left(y^{\prime}\right): y^{\prime} \in \varphi^{-1}(V \cup D)\right\}$.

(i) Suppose that $D^{\prime}$ is not empty. Let $x^{\prime} \in D^{\prime}$, and let $\Sigma_{1}$ be the minimal skeleton of $(X, D)$ containing $\Sigma \cup \varphi\left(\Sigma\left(X^{\prime}, V^{\prime} \cup D^{\prime} \backslash\left\{x^{\prime}\right\}\right)\right)$. Let $A^{\prime}$ be the connected component of $X^{\text {an }} \backslash\left(V^{\prime} \cup D^{\prime}\right)$ whose closure contains $x^{\prime}$, let $x=\varphi\left(x^{\prime}\right) \in D$, and let $A$ be the connected component of $X^{\text {an }} \backslash(V \cup D)$ whose closure contains $x$. Note that $\Sigma^{\prime}=\Sigma\left(X^{\prime}, V^{\prime} \cup D^{\prime} \backslash\left\{x^{\prime}\right\}\right) \cup \Sigma\left(A^{\prime}\right) \cup\left\{x^{\prime}\right\}$. Clearly, $\varphi\left(A^{\prime}\right) \subset A$, and $\varphi^{-1}(x) \cap A^{\prime}=\emptyset$ by construction. Since $\varphi$ takes the puncture of $A^{\prime}$ to the puncture of $A$, one shows as in the proof of Lemma 4.2 that $\varphi\left(\Sigma\left(A^{\prime}\right)\right) \subset \Sigma(A)$. Therefore, $\Sigma_{1}$ contains $\Sigma \cup \varphi\left(\Sigma^{\prime}\right)$.

(ii) Now, suppose that $D^{\prime}=\emptyset$ and $D \neq \emptyset$. Let $x \in D$, and let $\Sigma_{1}$ be the minimal skeleton of $(X, D \backslash\{x\})$ containing $\Sigma(X, V \cup D \backslash\{x\}) \cup \varphi\left(\Sigma^{\prime}\right)$. Let $\Sigma_{2}=\Sigma_{1} \cup[x, \tau(x)]$. It follows from Lemma 3.15(3) that $\Sigma_{2}$ is the minimal skeleton of $(X, D)$ containing $\Sigma_{1}$.

Let $V$ be a semistable vertex set of $(X, D)$. Recall from (3.11.1) that a connected component $C$ of $X^{\text {an }} \backslash(V \cup D)$ is adjacent to a vertex $x \in V$ provided that the closure of $C$ in $X^{\text {an }}$ contains $x$. Proposition 4.12(1) below is exactly [14], Theorem 4.5.3, when $D=D^{\prime}=\emptyset$.

Proposition 4.12. Suppose that $D^{\prime}=\varphi^{-1}(D)$ and that one of the following two conditions holds:

1. $\chi(X \backslash D) \leq 0$ (hence, also $\left.\chi\left(X^{\prime} \backslash D^{\prime}\right) \leq 0\right)$, or

2. $\varphi^{-1}(V) \subset V^{\prime}$.

In the situation of (1), let $\Sigma$ (resp. $\left.\Sigma^{\prime}\right)$ be the minimal skeleton of $(X, D)\left(\right.$ resp. $\left.\left(X^{\prime}, D^{\prime}\right)\right)$; and in (2), let $\Sigma=\Sigma(X, V \cup D)$ and $\Sigma^{\prime}=\Sigma\left(X^{\prime}, V^{\prime} \cup D^{\prime}\right)$. Then, $\varphi^{-1}(\Sigma) \subset \Sigma^{\prime}$.

Proof. First, suppose that (2) holds. Let $x^{\prime} \in X^{\prime \text { an }} \backslash \Sigma^{\prime}$, and let $B^{\prime}$ be the connected component of $X^{\prime \text { an }} \backslash \Sigma^{\prime}$ containing $x^{\prime}$, so $B^{\prime}$ is an open ball. By hypothesis, $\varphi\left(B^{\prime}\right)$ is contained in a connected component $C$ of $X^{\text {an }} \backslash(V \cup D)$. If $C$ is an open ball, then $\varphi\left(B^{\prime}\right) \cap \Sigma=\varnothing$ by the definition of $\Sigma(X, V \cup D)$. If $C$ is a generalized open annulus, then $\varphi\left(B^{\prime}\right) \cap \Sigma=\varphi\left(B^{\prime}\right) \cap \Sigma(C)=\emptyset$ by Lemma 4.3. Therefore, $\varphi\left(x^{\prime}\right) \notin \Sigma$.

Now, suppose that (1) holds. Let $V$ be a semistable vertex set of $(X, D)$ such that $\Sigma=$ $\Sigma(X, V \cup D)$. By subdividing edges of $\Sigma$ and enlarging $V$ if necessary, we may and do assume that $\Sigma$ has no loop edges. No point in $V$ of genus zero has valence one in $\Sigma$ : this follows from Proposition 3.18(2) if $\chi(X \backslash D)<0$, and from [12], Remark 4.24, if 
$\chi(X \backslash D)=0$. First, we claim that if $B^{\prime} \subset X^{\prime \text { an }} \backslash D^{\prime}$ is an open analytic domain which is isomorphic to $\mathbf{B}(1)_{+}$, then $\varphi\left(B^{\prime}\right) \cap V=\emptyset$. If $\varphi^{-1}(V) \cap B^{\prime}$ contains more than one point, then it easy to see that there exists a smaller open ball $B^{\prime \prime} \subset B^{\prime}$ such that $\varphi^{-1}(V) \cap B^{\prime \prime}$ contains exactly one point. Replacing $B^{\prime}$ by $B^{\prime \prime}$, we may assume that there is a unique point $y^{\prime} \in \varphi^{-1}(V) \cap B^{\prime}$. Let $y=\varphi\left(y^{\prime}\right) \in V$. Since $g\left(y^{\prime}\right)=0$, we have $g(y)=0$ by Lüroth's theorem.

The open analytic domain $B^{\prime} \backslash\left\{y^{\prime}\right\}$ is the disjoint union of an open annulus $A^{\prime}$ and an infinite collection of open balls by Lemma 3.4. Each connected component $C^{\prime}$ of $B^{\prime} \backslash\left\{y^{\prime}\right\}$ maps into a connected component $C$ of $X^{\text {an }} \backslash(V \cup D)$ adjacent to $y$, with the end $y^{\prime}$ of $C^{\prime}$ mapping to the end $y$ of $C$. By Lemma 4.3, no open ball connected component of $B^{\prime} \backslash\left\{y^{\prime}\right\}$ can map to a generalized open annulus connected component of $X^{\text {an }} \backslash(V \cup D)$. There are at least two generalized open annulus connected components $A$ of $X^{\text {an }} \backslash(V \cup D)$ adjacent to $y$, so some such $A$ must satisfy $\varphi\left(B^{\prime}\right) \cap A=\emptyset$. But the map $\varphi: X^{\prime \text { an }} \rightarrow X^{\text {an }}$ is open by [14], Lemma 3.2.4, so $\varphi\left(B^{\prime}\right)$ is an open neighborhood of $y$, which contradicts the fact that $y$ is a limit point of $A$. This proves the claim.

Let $V^{\prime}$ be a semistable vertex set of $\left(X^{\prime}, D^{\prime}\right)$ such that $\Sigma^{\prime}=\Sigma\left(X^{\prime}, V^{\prime} \cup D^{\prime}\right)$. Since $X^{\prime} \backslash \Sigma^{\prime}$ is a disjoint union of open balls, by the above, we have $\varphi^{-1}(V) \subset \Sigma^{\prime}$. Hence, we may enlarge $V^{\prime}$ to contain $\varphi^{-1}(V)$ without changing $\Sigma^{\prime}$, so we are reduced to (2).

Theorem 4.13. Let $\Sigma^{\prime}$ be a skeleton of $\left(X^{\prime}, D^{\prime}\right)$, and let $\Sigma$ be a skeleton of $(X, D)$. Suppose that $\varphi\left(\Sigma^{\prime}\right) \subset \Sigma$, and if $X \cong \mathbf{P}^{1}$ assume in addition that there exists a type-2 point $z \in \Sigma$ such that $\varphi^{-1}(z) \subset \Sigma^{\prime}$. Then, $\varphi^{-1}(\Sigma)$ is a skeleton of $\left(X^{\prime}, \varphi^{-1}(D)\right)$.

We will require the following lemmas. Lemma 4.14 is similar to [14], Corollary 4.5.4.

Lemma 4.14. Let $B^{\prime} \subset X^{\prime a n}$ be an open analytic domain isomorphic to $\mathbf{B}(1)_{+}$, and let $B=\varphi\left(B^{\prime}\right) \subset X^{a n}$. Then, $B$ is an open analytic domain of $X^{\text {an }}$, and one of the following is true:

1. $B$ is an open ball, and $\varphi: B^{\prime} \rightarrow B$ is finite and order-preserving.

2. $X \cong \mathbf{P}^{1}$, and $B=X^{\text {an }}$.

Proof. Suppose that the genus of $X$ is at least one, so $X^{\prime}$ also has genus at least one. By Lemma 4.6, we only need to show that $B$ is contained in an open ball in $X^{\text {an }}$. Let $\Sigma$ (resp. $\Sigma^{\prime}$ ) be the minimal skeleton of $X$ (resp. $X^{\prime}$ ). By Proposition 3.18(1), we have $B^{\prime} \subset X^{\prime \text { an }} \backslash \Sigma^{\prime}$; so by Proposition 4.12(1), we have $B \subset X^{\text {an }} \backslash \Sigma$. But every connected component of $X^{\text {an }} \backslash \Sigma$ is an open ball, so $B$ is contained in an open ball.

In the case $X=\mathbf{P}^{1}$, suppose first that $B^{\prime}(K) \rightarrow \mathbf{P}^{1}(K)$ is not surjective, so we may assume that $\infty \notin B$ after choosing a suitable coordinate on $\mathbf{P}^{1}$. Let $x^{\prime}$ be the end of $B^{\prime}$, and let $x=\varphi\left(x^{\prime}\right)$. Then, $x$ is a type- 2 point, so $\varphi\left(B^{\prime} \cup\left\{x^{\prime}\right\}\right)=B \cup\{x\}$ is a compact subset of $\mathbf{A}^{1 \text {,an }}$. Since $\mathbf{A}^{1 \text {,an }}$ is covered by an increasing union of open balls, $B$ is contained in an open ball.

Now, suppose that $B^{\prime}(K) \rightarrow \mathbf{P}^{1}(K)$ is surjective. Since $\mathbf{P}^{1}(K)$ is dense in $\mathbf{P}^{1}$, an and $B \cup\{x\}$ is closed in $\mathbf{P}^{1 \text {,an }}$, it follows that $B \cup\{x\}=\mathbf{P}^{1 \text {,an }}$. If $x \notin B$, then $B$ is contained in a connected component of $\mathbf{P}^{1, \text { an }} \backslash\{x\}$, which contradicts the surjectivity of $B^{\prime}(K) \rightarrow \mathbf{P}^{1}(K)$. Therefore, $B=\mathbf{P}^{1, \text { an }}$. 
Remark 4.15. Case (2) of Lemma 4.14 does occur. For instance, let $\varphi: \mathbf{P}^{1} \rightarrow \mathbf{P}^{1}$ be the finite morphism $t \mapsto t^{2}$, and let $B^{\prime}$ be the open ball in $\mathbf{P}^{1, \text { an }}$ obtained by deleting the closed ball of radius $1 / 2$ around $1 \in \mathbf{P}^{1}(K)$. If $\operatorname{char}(k) \neq 2$, then for every point $x \in \mathbf{P}^{1}(K)$, either $x \in B^{\prime}(K)$ or $-x \in B^{\prime}(K)$, so $B^{\prime} \rightarrow \mathbf{P}^{1, \text { an }}$ is surjective.

Lemma 4.16. Let $V$ be a semistable vertex set of $(X, D)$, let $\Sigma=\Sigma(X, V \cup D)$, and let $B \subset X$ be an open analytic domain isomorphic to $\mathbf{B}(1)+$ whose end $x$ is contained in $\Sigma$. Then,

$$
\Sigma \cap \bar{B}=\bigcup_{y \in B \cap(V \cup D)}[x, y]
$$

Proof. We may assume without loss of generality that $x \in V$. Let $V_{0}=V \backslash(V \cap$ $B)$, and $D_{0}=D \backslash(D \cap B)$. Then, $V_{0}$ is a semistable vertex set of $\left(X, D_{0}\right)$ : indeed, every connected component of $X^{\text {an }} \backslash(V \cup D)$ is either a connected component of $X^{\text {an }} \backslash\left(V_{0} \cup D_{0}\right)$ or is contained in $B$, so $X^{\text {an }} \backslash\left(V_{0} \cup D_{0}\right)$ is a disjoint union of open balls and finitely many generalized open annuli. Let $\Sigma_{0}=\Sigma\left(X, V_{0} \cup D_{0}\right)$, so $x \in \Sigma_{0}$ but $B \cap \Sigma_{0}=\emptyset$ by Proposition 3.12(1). Since $B$ lies in a connected component of $X^{\text {an }} \backslash \Sigma_{0}$, which has to be an open ball, and since the end of $B$ is contained in $\Sigma_{0}$, by Lemma 4.6, we have that $B$ is in fact a connected component of $X^{\text {an }} \backslash \Sigma_{0}$, so the Lemma now follows from Lemma 3.15 as applied to $\Sigma=\Sigma_{0}, W=V \cap B$, and $E=D \cap B$.

Proof. Note that $\varphi\left(\Sigma^{\prime}\right) \subset \Sigma$ implies $\varphi\left(D^{\prime}\right) \subset D$. Let $V$ (resp. $V^{\prime}$ ) be a vertex set for $\Sigma$ (resp. $\Sigma^{\prime}$ ). We claim that

$$
\varphi^{-1}(\Sigma)=\Sigma^{\prime} \cup \bigcup_{x^{\prime} \in \varphi^{-1}(V \cup D)}\left[x^{\prime}, \tau_{\Sigma^{\prime}}\left(x^{\prime}\right)\right]
$$

which by Lemma 3.15 is the minimal skeleton of $\left(X^{\prime}, \varphi^{-1}(D)\right)$ containing $\Sigma^{\prime}$ and $\varphi^{-1}(V)$.

Let $B^{\prime}$ be a connected component of $X^{\prime a n} \backslash \Sigma^{\prime}$, let $x^{\prime} \in \Sigma^{\prime}$ be its end, and let $x=\varphi\left(x^{\prime}\right) \in$ $\Sigma$. Then, $B=\varphi\left(B^{\prime}\right)$ is an open ball and $x$ is its end: if $X ¥ \mathbf{P}^{1}$, then this follows directly from Lemma 4.14; and if $X \cong \mathbf{P}^{1}$, then $B \subset X^{\text {an }} \backslash\{z\}$, so $\varphi(B) \neq X^{\text {an }}$ and therefore $B$ is an open ball in this case as well. Hence,

$$
\Sigma \cap B=\bigcup_{y \in B \cap(V \cup D)}[x, y]
$$

by Lemma 4.16, so

$$
\varphi^{-1}(\Sigma) \cap B^{\prime}=\bigcup_{y^{\prime} \in B^{\prime} \cap \varphi^{-1}(V \cup D)}\left[x^{\prime}, y^{\prime}\right]
$$

by Proposition 4.8 .

Remark 4.17. When $X \cong \mathbf{P}^{1}$, the extra hypothesis on $\Sigma^{\prime}$ in Theorem 4.13 is necessary. Indeed, let $\varphi: \mathbf{P}^{1} \rightarrow \mathbf{P}^{1}$ be a finite morphism and let $x^{\prime} \in \mathbf{P}^{1, \text { an }}$ be a type-2 point such that $\varphi^{-1}\left(\varphi\left(x^{\prime}\right)\right)$ has more than one element. Then, $\Sigma=\left\{\varphi\left(x^{\prime}\right)\right\}$ is a skeleton containing the image of the skeleton $\Sigma^{\prime}=\left\{x^{\prime}\right\}$, but $\varphi^{-1}(\Sigma)$ is not a skeleton. 
Corollary 4.18. Let $\Sigma$ (resp. $\left.\Sigma^{\prime}\right)$ be a skeleton of $(X, D)$ (resp. $\left.\left(X^{\prime}, D^{\prime}\right)\right)$. There exists a skeleton $\Sigma_{1}$ of $\left(X, D \cup \varphi\left(D^{\prime}\right)\right)$ such that $\Sigma_{1} \supset \Sigma \cup \varphi\left(\Sigma^{\prime}\right)$ and such that $\varphi^{-1}\left(\Sigma_{1}\right)$ is a skeleton of $\left(X^{\prime}, \varphi^{-1}\left(D \cup \varphi\left(D^{\prime}\right)\right)\right)$. Moreover, there is a minimal such $\Sigma_{1}$ with respect to inclusion.

Proof. If $X \nsubseteq \mathbf{P}^{1}$, then this is an immediate consequence of Proposition 4.11 and Theorem 4.13: if $\Sigma_{1}$ is the minimal skeleton of $\left(X, D \cup \varphi\left(D^{\prime}\right)\right)$ containing $\Sigma \cup \varphi\left(\Sigma^{\prime}\right)$, then $\varphi^{-1}\left(\Sigma_{1}\right)$ is a skeleton of $\left(X^{\prime}, \varphi^{-1}\left(D \cup \varphi\left(D^{\prime}\right)\right)\right)$. Suppose then that $X \cong \mathbf{P}^{1}$. Let $x^{\prime} \in \Sigma^{\prime}$ be a type-2 point, let $x=\varphi\left(x^{\prime}\right)$, and let $x^{\prime}, x_{1}^{\prime}, x_{2}^{\prime}, \ldots, x_{n}^{\prime}$ be the points of $X^{\prime}$ mapping to $x$. Let

$$
\Sigma_{1}^{\prime}=\Sigma^{\prime} \cup \bigcup_{i=1}^{n}\left[x_{i}^{\prime}, \tau_{\Sigma^{\prime}}\left(x_{i}^{\prime}\right)\right] .
$$

This is the minimal skeleton of $\left(X^{\prime}, D^{\prime}\right)$ containing $\Sigma^{\prime}$ and $\varphi^{-1}(x)$ by Lemma 3.15(3). Let $\Sigma_{1}$ be the minimal skeleton of $\left(X, D \cup \varphi\left(D^{\prime}\right)\right)$ containing $\Sigma \cup \varphi\left(\Sigma_{1}^{\prime}\right)$. Then, $\varphi^{-1}\left(\Sigma_{1}\right)$ is a skeleton of $\left(X^{\prime}, \varphi^{-1}\left(D \cup \varphi\left(D^{\prime}\right)\right)\right)$ by Theorem 4.13. If $\Sigma_{2}$ is a skeleton of $\left(X, D \cup \varphi\left(D^{\prime}\right)\right)$ such that $\Sigma_{2} \supset \Sigma \cup \varphi\left(\Sigma^{\prime}\right)$ and $\varphi^{-1}\left(\Sigma_{2}\right)$ is a skeleton of $\left(X^{\prime}, \varphi^{-1}\left(D \cup \varphi\left(D^{\prime}\right)\right)\right)$, then $\varphi^{-1}\left(\Sigma_{2}\right)$ contains each geodesic $\left[x_{i}^{\prime}, \tau_{\Sigma^{\prime}}\left(x_{i}^{\prime}\right)\right]$, so $\varphi^{-1}\left(\Sigma_{2}\right)$ contains $\Sigma_{1}^{\prime}$ and therefore $\Sigma_{2} \supset \Sigma_{1}$.

Remark 4.19. Suppose that $\varphi: X^{\prime} \rightarrow X$ is a finite morphism such that $\varphi^{-1}(D)=D^{\prime}$ and $\varphi^{-1}(V)=V^{\prime}$. Let $\Sigma=\Sigma(X, V \cup D)$, and $\Sigma^{\prime}=\Sigma\left(X^{\prime}, V^{\prime} \cup D^{\prime}\right)$. In this case, we can describe the skeleton $\Sigma_{1}$ of Corollary 4.18 more explicitly, as follows. Let $e^{\prime}$ be an open edge of $\Sigma^{\prime}$ with respect to the given choice of vertex set, and let $A^{\prime}=\tau^{-1}\left(e^{\prime}\right)$. This is a connected component of $X^{\text {an }} \backslash\left(V^{\prime} \cup D^{\prime}\right)$. Let $A$ be the connected component of $X^{\text {an }} \backslash(V \cup D)$ containing $\varphi\left(A^{\prime}\right)$. Since $A^{\prime}$ is a connected component of $\varphi^{-1}(A)$, the map $A^{\prime} \rightarrow A$ is finite, hence surjective. If $A^{\prime}$ is a punctured open ball, then so is $A$; so by Lemma 4.2, $\varphi$ maps $e^{\prime}$ homeomorphically with nonzero integer expansion factor onto $e=\Sigma(A)$. If $A^{\prime}$ is an open annulus, then $A$ could be an open annulus or an open ball; in the former case, we again have $e^{\prime}$ mapping homeomorphically onto $e=\Sigma(A)$ with nonzero integer expansion factor. If $A$ is an open ball, then by Proposition 4.9, $e^{\prime}$ maps onto a geodesic segment in $A$; clearly both vertices of $e^{\prime}$ map to the end of $A$, so $e^{\prime}$ 'goes straight and doubles back' under $\varphi$.

It follows from this and Proposition 4.12(2) that $\varphi\left(\Sigma^{\prime}\right)$ is equal to $\Sigma$ union a finite number of geodesic segments $T_{1}, \ldots, T_{r}$ contained in open balls $B_{1}, \ldots, B_{r} \subset X^{\text {an }} \backslash(V \cup D)$ and with one endpoint at elements of $V$. These are precisely the images of the edges of $\Sigma^{\prime}$ not mapping to edges of $\Sigma$. By Lemma 3.15, $\Sigma \cup\left(T_{1} \cup \cdots \cup T_{r}\right)$ is again a skeleton of $X$, so in this case

$$
\Sigma_{1}=\varphi\left(\Sigma^{\prime}\right)=\Sigma \cup\left(T_{1} \cup \cdots \cup T_{r}\right)
$$

(the inverse image of $\Sigma_{1}$ is a skeleton by Theorem 4.13).

\subsection{Tangent directions and morphisms of curves}

Our next goal is to prove that a finite morphism of curves induces a finite harmonic morphism of metrized complexes of curves for a suitable choice of skeleta. First, we need to formulate and prove a relative version of Theorem 3.21. Let $X, X^{\prime}$ be smooth, proper, connected $K$-curves. 
Definition 4.21. A continuous function $\varphi: X^{\prime a n} \rightarrow X^{\text {an }}$ is piecewise affine provided that, for every geodesic segment $\alpha:[a, b] \hookrightarrow X^{\prime a n}$, the composition $\varphi \circ \alpha$ is piecewise affine with integer expansion factors in the sense of Definition 4.4.

Let $\varphi: X^{\prime \text { an }} \rightarrow X^{\text {an }}$ be a piecewise affine function, let $x^{\prime} \in X^{\prime \text { an }}$, and let $x=\varphi\left(x^{\prime}\right) \in X^{\text {an }}$. Let $v^{\prime} \in T_{x^{\prime}}$, and let $\alpha:[a, b] \hookrightarrow X^{\prime \text { an }}$ be a geodesic segment representing $v^{\prime}$ (so $a=-\infty$ if $x^{\prime}$ has type 1). Taking $b$ small enough, we can assume that $\varphi \circ \alpha$ is an embedding with integer expansion factor $m$. Let $v \in T_{x}$ be the tangent direction represented by $\varphi \circ \alpha$. We define

$$
d \varphi\left(x^{\prime}\right): T_{x^{\prime}} \rightarrow T_{x} \text { by } d \varphi\left(x^{\prime}\right)\left(v^{\prime}\right)=v ;
$$

this is independent of the choice of $\alpha$. We call the expansion factor $m$ the outgoing slope of $\varphi$ in the direction $v^{\prime}$, and we write $m=d_{v^{\prime}} \varphi\left(x^{\prime}\right)$.

Definition 4.22. A piecewise affine function $\varphi: X^{\prime a n} \rightarrow X^{\text {an }}$ is harmonic at a point $x^{\prime} \in X^{\prime a n}$ provided that, for all $v \in T_{\varphi\left(x^{\prime}\right)}$, the sum

$$
\sum_{\substack{\nu^{\prime} \in T_{x^{\prime}} \\ d \varphi\left(v^{\prime}\right)=v}} d_{\nu^{\prime}} \varphi\left(x^{\prime}\right)
$$

has a finite number of nonzero terms and is independent of the choice of $v$.

Let $\varphi: X^{\prime} \rightarrow X$ be a finite morphism of smooth, proper, connected $K$-curves, let $x^{\prime} \in X^{\prime a n}$ be a type-2 point, and let $x=\varphi\left(x^{\prime}\right)$. Let $C_{x}$ and $C_{x^{\prime}}$ denote the smooth proper connected $k$-curves with function fields $\widetilde{\mathscr{H}}(x)$ and $\widetilde{\mathscr{H}}\left(x^{\prime}\right)$, respectively. We denote by $\varphi_{x^{\prime}}$ the induced morphism $C_{x^{\prime}} \rightarrow C_{x}$. We have the following relative version of the slope formula of Theorem 3.21:

Theorem 4.23. Let $\varphi: X^{\prime} \rightarrow X$ be a finite morphism of smooth, proper, connected K-curves.

1. The analytification $\varphi: X^{\prime a n} \rightarrow X^{\text {an }}$ is piecewise affine and harmonic.

2. Let $x^{\prime} \in X^{\prime a n}$ be a type-2 point, let $x=\varphi\left(x^{\prime}\right)$, let $v^{\prime} \in T_{x^{\prime}}$, let $v=d \varphi\left(v^{\prime}\right) \in T_{x}$, and let $\xi_{v^{\prime}} \in C_{x^{\prime}}$ and $\xi_{v} \in C_{x}$ be the closed points associated Equation 3.22.1 to $v^{\prime}$ and $v$, respectively. Then, $\varphi_{x^{\prime}}\left(\xi_{v^{\prime}}\right)=\xi_{v}$, and the ramification degree of $\varphi_{x^{\prime}}$ at $\xi_{v^{\prime}}$ is equal to $d_{v^{\prime}} \varphi\left(x^{\prime}\right)$.

3. Let $x^{\prime} \in X^{\prime a n}$ be a type-1 point, and let $v^{\prime} \in T_{x^{\prime}}$ be the unique tangent direction. Then, $d_{v^{\prime}} \varphi\left(x^{\prime}\right)$ is the ramification degree of $\varphi$ at $x^{\prime}$.

Proof. First we claim that $\varphi$ is piecewise affine. Let $\left[x^{\prime}, y^{\prime}\right] \subset X^{\prime \text { an }}$ be a geodesic segment. Suppose first that $x^{\prime}$ and $y^{\prime}$ have type 2 or 3 . Then, there exists a skeleton $\Sigma^{\prime}$ of $X^{\prime}$ containing $\left[x^{\prime}, y^{\prime}\right]$ by [12], Corollaries 5.1 and 5.10 . From this, we easily reduce to the case that $\left[x^{\prime}, y^{\prime}\right]$ is an edge of $\Sigma^{\prime}$ with respect to some vertex set; now, the claim follows from Lemma 4.2 and Proposition 4.9 as in the proof of Proposition 4.11 (which provides a finite subdivision of $\left[x^{\prime}, y^{\prime}\right]$ into closed geodesic segments $I_{j}$ such that the image of the interior of each $I_{j}$ lies either in an open annulus or an open ball). If $x^{\prime}$ has type 1 or 4 , then there is an open neighborhood $B$ of $x=\varphi\left(x^{\prime}\right)$ and an open neighborhood $B^{\prime} \subset \varphi^{-1}(B)$ of $x^{\prime}$ such that $B$ and $B^{\prime}$ are open balls. Shrinking $B^{\prime}$ if necessary, we can assume that $y^{\prime} \notin B^{\prime}$, so the 
end $z^{\prime}$ of $B^{\prime}$ is contained in $\left[x^{\prime}, y^{\prime}\right]$ (removing $z^{\prime}$ disconnects $X^{\prime a n}$ ). The restriction of $\varphi$ to $\left[x^{\prime}, z^{\prime}\right]$ is piecewise affine by Lemma 4.5 , so this proves the claim.

Next, we prove (2). By functoriality of the reduction map, for any rational function $f$ on $X$ such that $|f(x)|=1$, we have $\varphi_{x^{\prime}}^{*}\left(\widetilde{f}_{x}\right)=\widetilde{\varphi^{*}(f)_{x^{\prime}}}$. By the slope formula (Theorem 3.21), the point $\xi_{v}$ corresponds to the discrete valuation on $\widetilde{\mathscr{H}}(x)$ given by $\operatorname{ord}_{\xi_{v}}\left(\tilde{f}_{x}\right)=d_{v} F(x)$, where $F=-\log |f|$. Similarly, the slope formula applied to $\varphi^{*}(f)$ on $X^{\prime}$ gives ord $\xi_{\nu^{\prime}}\left(\widetilde{\varphi^{*}(f)_{x^{\prime}}}\right)=d_{\nu^{\prime}} \varphi\left(x^{\prime}\right) d_{\nu} F(x)$. Therefore, we get

$$
\operatorname{ord}_{\xi_{v^{\prime}}}\left(\varphi_{x^{\prime}}^{*}\left(\widetilde{f}_{x}\right)\right)=d_{v^{\prime}} \varphi\left(x^{\prime}\right) \operatorname{ord}_{\xi_{v}}\left(\tilde{f}_{x}\right) .
$$

Since any non-zero element of $\widetilde{\mathscr{H}}(x)$ is of the form $\widetilde{f}_{x}$ for some rational function $f$ on $X$ with $|f(x)|=1$, this shows that the center of the valuation $\xi_{v^{\prime}}$ on $\widetilde{\mathscr{H}}(x) \stackrel{\varphi_{x^{\prime}}^{*}}{\longleftrightarrow} \widetilde{\mathscr{H}}\left(x^{\prime}\right)$ coincides with the center of $\xi_{v}$, that is $\varphi_{x^{\prime}}\left(\xi_{v^{\prime}}\right)=\xi_{v}$, and at the same time, the ramification degree of $\varphi_{x^{\prime}}$ at $\xi_{v^{\prime}}$ is equal to $d_{v^{\prime}} \varphi\left(x^{\prime}\right)$.

The proof of (3) proceeds in the same way as (2), applying the slope formula to a nonzero rational function $f$ on $X$ with a zero at $x=\varphi\left(x^{\prime}\right)$ and to $\varphi^{*}(f)$ on $X^{\prime}$.

\subsection{Morphisms of curves induce morphisms of metrized complexes}

More precisely, this occurs when the morphism of curves respects a choice of triangulations, in the following sense.

Definition 4.25. Let $(X, V \cup D)$ and $\left(X^{\prime}, V^{\prime} \cup D^{\prime}\right)$ be triangulated punctured curves. A finite morphism $\varphi:\left(X^{\prime}, V^{\prime} \cup D^{\prime}\right) \rightarrow(X, V \cup D)$ is a finite morphism $\varphi: X^{\prime} \rightarrow X$ such that $\varphi^{-1}(D)=D^{\prime}, \varphi^{-1}(V)=V^{\prime}$, and $\varphi^{-1}(\Sigma(X, V \cup D))=\Sigma\left(X^{\prime}, V^{\prime} \cup D^{\prime}\right)$ (as sets).

Corollary 4.26. Let $\varphi: X^{\prime} \rightarrow X$ be a finite morphism of smooth, connected, proper $K$-curves, let $D \subset X(K)$ be a finite set, and let $D^{\prime}=\varphi^{-1}(D)$. There exists a strongly semistable vertex set $V$ of $(X, D)$ such that $V^{\prime}=\varphi^{-1}(V)$ is a strongly semistable vertex set of $\left(X^{\prime}, D^{\prime}\right)$ and such that

$$
\Sigma\left(X^{\prime}, V^{\prime} \cup D^{\prime}\right)=\varphi^{-1}(\Sigma(X, V \cup D)) .
$$

In particular, $\varphi$ extends to a finite morphism of triangulated punctured curves.

Proof. By Corollary 4.18, there is a skeleton $\Sigma$ of $(X, D)$ such that $\Sigma^{\prime}=\varphi^{-1}(\Sigma)$ is a skeleton of $\left(X^{\prime}, D^{\prime}\right)$. Let $V_{0}^{\prime}$ be a strongly semistable vertex set of $\Sigma^{\prime}$, let $V$ be a strongly semistable vertex set of $\Sigma$ containing $\varphi\left(V_{0}^{\prime}\right)$, and let $V^{\prime}=\varphi^{-1}(V)$. Then, $V^{\prime}$ is again a strongly semistable vertex set of $\Sigma^{\prime}$.

Let $\varphi:\left(X^{\prime}, V^{\prime} \cup D^{\prime}\right) \rightarrow(X, V \cup D)$ be a finite morphism of triangulated punctured curves. Let $\Sigma=\Sigma(X, V \cup D)$, and $\Sigma^{\prime}=\Sigma\left(X^{\prime}, V^{\prime} \cup D^{\prime}\right)$. Let $e^{\prime}$ be an open edge of $\Sigma^{\prime}$, and let $e=\varphi\left(e^{\prime}\right)$, an open edge of $\Sigma$. The image of the annulus $A^{\prime}=\tau^{-1}\left(e^{\prime}\right)$ is contained in $A=\tau^{-1}(e)$, so the restriction of $\varphi$ to $e^{\prime}$ is an embedding with integer expansion factor by Lemma 4.2(2). It is clear from this and Theorem 4.23 that $\left.\varphi\right|_{\Sigma^{\prime}}: \Sigma^{\prime} \rightarrow \Sigma$ is a harmonic $\left(V^{\prime}, V\right)$-morphism of $\Lambda$-metric graphs. For $x^{\prime} \in V^{\prime}$ with image $x=\varphi\left(x^{\prime}\right)$, we have a finite morphism of $k$-curves $\varphi_{x^{\prime}}: C_{x^{\prime}} \rightarrow C_{x}$. It now follows from Theorem 4.23 that these 
extra data enrich $\left.\varphi\right|_{\Sigma^{\prime}}: \Sigma^{\prime} \rightarrow \Sigma$ with the structure of a finite harmonic morphism of $\Lambda$-metrized complexes of curves:

Corollary 4.28. Let $\varphi:\left(X^{\prime}, V^{\prime} \cup D^{\prime}\right) \rightarrow(X, V \cup D)$ be a finite morphism of triangulated punctured curves. Then $\varphi$ naturally induces a finite harmonic morphism of $\Lambda$-metrized complexes of curves

$$
\Sigma\left(X^{\prime}, V^{\prime} \cup D^{\prime}\right) \longrightarrow \Sigma(X, V \cup D)
$$

Remark 4.29. More generally, suppose that $(X, V \cup D)$ and $\left(X^{\prime}, V^{\prime} \cup D^{\prime}\right)$ are triangulated punctured $K$-curves and that $\varphi: X^{\prime} \rightarrow X$ is a finite morphism such that $\varphi^{-1}(D)=D^{\prime}$ and $\varphi^{-1}(V) \subset V^{\prime}$. Then, $\varphi^{-1}(\Sigma) \subset \Sigma^{\prime}$ by Proposition 4.12(2). One can show using Theorem 4.23 that the map $\Sigma^{\prime} \rightarrow X^{\text {an }}$ followed by the retraction $X^{\text {an }} \rightarrow \Sigma$ is a (not necessarily finite) harmonic morphism of metric graphs $\Sigma^{\prime} \rightarrow \Sigma$.

\subsection{Tame coverings of triangulated curves}

Many of the results in this paper involve constructing a curve $X^{\prime}$ and a morphism $\varphi$ : $X^{\prime} \rightarrow X$ inducing a given morphism of skeleta as in Corollary 4.28. For these purposes, it is useful to introduce some mild restrictions on the morphism $\varphi$. Fix a triangulated punctured curve $(X, V \cup D)$ with skeleton $\Sigma=\Sigma(X, V \cup D)$, regarded as a metrized complex of curves as in (3.22).

Definition 4.31. Let $\left(X^{\prime}, V^{\prime} \cup D^{\prime}\right)$ be a triangulated punctured curve with skeleton $\Sigma^{\prime}=$ $\Sigma\left(X^{\prime}, V^{\prime} \cup D^{\prime}\right)$, and let $\varphi:\left(X^{\prime}, V^{\prime} \cup D^{\prime}\right) \rightarrow(X, V \cup D)$ be a finite morphism. We say that $\varphi$ is a tame covering of $(X, V \cup D)$ provided that:

1. $D$ contains the branch locus of $\varphi$,

2. if $\operatorname{char}(k)=p>0$, then for every edge $e^{\prime} \in E\left(\Sigma^{\prime}\right)$ the expansion factor $d_{e^{\prime}}(\varphi)$ is not divisible by $p$, and

3. $\varphi_{x^{\prime}}$ is separable (= generically étale) for all $x^{\prime} \in V^{\prime}$.

\section{Remark 4.32.}

1. Since $D$ is finite, it follows from (1) and Theorem 4.23(3) that a tame covering of $(X, V \cup D)$ is a tamely ramified morphism of curves.

2. If $\Sigma$ has at least one edge, then (2) implies (3).

3. Let $\varphi:\left(X^{\prime}, V^{\prime} \cup D^{\prime}\right) \rightarrow(X, V \cup D)$ be a tame covering, let $S \subset \Sigma$ be a finite set of type-2 points, and let $S^{\prime}=\varphi^{-1}(S)$. Then $S^{\prime} \subset \Sigma\left(X^{\prime}, V^{\prime} \cup D^{\prime}\right)$ is also a finite set of type-2 points, so $\varphi$ also defines a tame covering $\left(X^{\prime}, S^{\prime} \cup V^{\prime} \cup D^{\prime}\right) \rightarrow(X, S \cup V \cup D)$.

Lemma 4.33. Let $\varphi:\left(X^{\prime}, V^{\prime} \cup D^{\prime}\right) \rightarrow(X, V \cup D)$ be a tame covering, and let $\Sigma=$ $\Sigma(X, V \cup D)$ and $\Sigma^{\prime}=\Sigma\left(X^{\prime}, V^{\prime} \cup D^{\prime}\right)$. Then, $\left.\varphi\right|_{\Sigma^{\prime}}: \Sigma^{\prime} \rightarrow \Sigma$ is a tame covering of metrized complexes of curves.

Proof. Let $\Gamma$ (resp. $\Gamma^{\prime}$ ) be the augmented metric graph underlying $\Sigma$ (resp. $\Sigma^{\prime}$ ). By Proposition 2.22, we only have to show that $\left.\varphi\right|_{\Gamma^{\prime}}$ is generically étale. Let $R=$ $\sum_{x^{\prime} \in V^{\prime} \cup D^{\prime}} R_{x^{\prime}}\left(x^{\prime}\right)$ be the ramification divisor of $\left.\varphi\right|_{\Gamma^{\prime}}$ as defined in Equation 2.14.1, and let 
$S=\sum_{x^{\prime} \in D^{\prime}} S_{x^{\prime}}\left(x^{\prime}\right)$ be the ramification divisor of $\varphi: X^{\prime} \rightarrow X$. We will show that $R=S$. Since $\varphi_{x^{\prime}}$ is generically étale for all $x^{\prime} \in V^{\prime}$, we see from Equation 2.14.1 and the RiemannHurwitz formula as applied to $\varphi_{x^{\prime}}$ that $R_{x^{\prime}} \geq 0$ for $x^{\prime} \in V^{\prime}$. If $x^{\prime} \in D^{\prime}$, then there is a unique edge $e^{\prime}$ adjacent to $x^{\prime}$ and $d_{e^{\prime}}(\varphi)=d_{x^{\prime}}(\varphi)$, so

$$
R_{x^{\prime}}=2 d_{x^{\prime}}(\varphi)-2-d_{x^{\prime}}(\varphi)+1=d_{x^{\prime}}(\varphi)-1=S_{x^{\prime}},
$$

where the final equality is Theorem 4.23(3). Since $R_{x^{\prime}} \geq 0$ for all $x^{\prime} \in V^{\prime}$, it is enough to show that $\operatorname{deg}(R)=\operatorname{deg}(S)$. By Equation 2.14.2, we have $K_{\Gamma^{\prime}}=\left(\left.\varphi\right|_{\Gamma^{\prime}}\right)^{*}\left(K_{\Gamma}\right)+R$, so counting degrees gives

$$
\operatorname{deg}(R)=\operatorname{deg}\left(\left.\varphi\right|_{\Gamma^{\prime}}\right)(2-2 g(\Gamma))-\left(2-2 g\left(\Gamma^{\prime}\right)\right) .
$$

The Lemma now follows from the equalities $\operatorname{deg}(\varphi)=\operatorname{deg}\left(\left.\varphi\right|_{\Gamma^{\prime}}\right), g(\Gamma)=g(X), g\left(\Gamma^{\prime}\right)=$ $g\left(X^{\prime}\right)$, and the Riemann-Hurwitz formula as applied to $\varphi: X^{\prime} \rightarrow X$.

Remark 4.34. We showed in the proof of Lemma 4.33 that the ramification divisor of $\left.\varphi\right|_{\Gamma^{\prime}}$ coincides with the ramification divisor of $\varphi$. Moreover, it follows from Proposition 2.22 that for every $x^{\prime} \in V^{\prime}$, every ramification point $\vec{x} \in C_{x^{\prime}}$ is the reduction of a tangent direction in $\Gamma^{\prime}$. In other words, for a tame covering all ramification points of all residue curves are 'visible' in the morphism of underlying metric graphs $\Gamma^{\prime} \rightarrow \Gamma$.

Proposition 4.35. Let $\varphi:\left(X^{\prime}, V^{\prime} \cup D^{\prime}\right) \rightarrow(X, V \cup D)$ be a finite morphism of triangulated punctured curves. Then, $\varphi$ is a tame covering if and only if

1. D contains the branch locus of $\varphi$ and

2. $\varphi_{x^{\prime}}: C_{x^{\prime}} \rightarrow C_{\varphi(x)}$ is tamely ramified for every type-2 point $x^{\prime} \in X^{\prime a n}$.

Moreover, if $\varphi$ is a tame covering and $x^{\prime} \in X^{\prime a n}$ is a type-2 point not contained in $\Sigma^{\prime}$, then $\varphi$ is an isomorphism in a neighborhood of $x^{\prime}$.

Proof. It is clear that conditions (1) and (2) imply that $\varphi$ is a tame covering, so suppose that $\varphi$ is a tame covering and $x^{\prime} \in X^{\prime a n}$ is a type- 2 point. We have the following cases:

- If $x^{\prime} \in V^{\prime}$, then $\varphi_{x^{\prime}}$ is tamely ramified by Lemma 4.33 and Proposition 2.22.

- Suppose that $x^{\prime}$ is contained in the interior of an edge $e^{\prime}$ of $\Sigma^{\prime}$. Since $\varphi$ is a tame covering of $\left(X,\left(V \cup\left\{\varphi\left(y^{\prime}\right)\right\}\right) \cup D\right)$ by Remark 4.32(3), we are reduced to the case treated above.

- Suppose that $x^{\prime} \notin \Sigma^{\prime}$ but $y^{\prime}=\tau\left(x^{\prime}\right)$ is contained in $V^{\prime}$. Let $\mathfrak{X}$ (resp. $\mathfrak{X}^{\prime}$ ) be the semistable formal model of $X$ (resp. $X^{\prime}$ ) corresponding to the semistable vertex set $V$ (resp. $V^{\prime}$ ), and let $\varphi$ denote the unique finite morphism $\mathfrak{X}^{\prime} \rightarrow \mathfrak{X}$ extending $\varphi: X^{\prime} \rightarrow X$ (see Theorem 5.13). Let $\bar{x}^{\prime}=\operatorname{red}\left(x^{\prime}\right) \in \mathfrak{X}^{\prime}(k)$, and let $x=\varphi\left(x^{\prime}\right)$ and $\bar{x}=\operatorname{red}(x)=\varphi_{k}\left(\bar{x}^{\prime}\right)$. The connected component $B^{\prime}$ of $x^{\prime}$ in $X^{\prime a n} \backslash \Sigma^{\prime}$ is equal to $\operatorname{red}^{-1}\left(\bar{x}^{\prime}\right)$, and the connected component $B$ of $x$ in $X^{\text {an }} \backslash \Sigma$ is equal to red ${ }^{-1}(\bar{x})$. Since $\Sigma \cap B=\emptyset, \varphi_{k}$ is not branched over $\bar{x}$ by Lemma 4.33 and Proposition 2.22. Therefore, $\varphi^{-1}(B)$ is a disjoint union of $\operatorname{deg}(\varphi)$ open balls (one of which is $B^{\prime}$ ) mapping isomorphically onto $B$. In particular, $\varphi$ is an isomorphism in a neighborhood of $x^{\prime}$.

- Suppose that $x^{\prime} \notin \Sigma^{\prime}$ but $y^{\prime}=\tau\left(x^{\prime}\right)$ is contained in the interior of an edge of $\Sigma^{\prime}$. We claim that $\varphi$ is an isomorphism in a neighborhood of $x^{\prime}$. Let $y=\varphi\left(y^{\prime}\right) \in \Sigma$. Then, $\varphi$ is 
a tame covering of $(X,(V \cup\{y\}) \cup D)$, so replacing $V$ with $V \cup\{y\}$ and $V^{\prime}$ with $V^{\prime} \cup \varphi^{-1}(y)$ (see Remark 4.32(3)), we are reduced to the previous case.

Remark 4.36. It follows from Proposition 4.35 that if $\varphi$ is a tame covering, then the set-theoretic branch locus of $\varphi: X^{\prime a n} \rightarrow X^{\text {an }}$ (i.e. the set of all points in $X^{\text {an }}$ with fewer than $\operatorname{deg}(\varphi)$ points in its fiber) is contained in $\Sigma$. See $[27,28]$ for more on the topic of the Berkovich ramification locus in the case of self-maps of $\mathbf{P}^{1}$.

\section{Applications to morphisms of semistable models}

In this section, we show how Section 4 formally implies a large part of the results of Liu [34] on simultaneous semistable reduction of morphisms of curves (see also [23]) over an arbitrary valued field. In addition to establishing these results over a more general ground field, we feel that the 'skeletal' point of view on simultaneous semistable reduction is enlightening (see Remark 5.23). To this end, we let $K_{0}$ be any field equipped with a nontrivial non-Archimedean valuation val $: K_{0} \rightarrow \mathbf{R} \cup\{\infty\}$. Its valuation ring will be denoted $R_{0}$, its maximal ideal $\mathfrak{m}_{R_{0}}$, and its residue field $k_{0}$.

Let $X$ be a smooth, proper, geometrically connected algebraic $K_{0}$-curve. By a (strongly) semistable $R_{0}$-model of $X$, we mean a flat, integral, proper relative curve $\mathcal{X} \rightarrow \operatorname{Spec}\left(R_{0}\right)$ whose special fiber $\mathcal{X}_{k_{0}}$ is a (strongly) semistable curve (i.e. $\mathcal{X}_{\bar{k}_{0}}$ is a reduced curve with at worst ordinary double point singularities; it is strongly semistable if its irreducible components are smooth) and whose generic fiber is equipped with an isomorphism $\mathcal{X}_{K_{0}} \cong X$. By properness of $\mathcal{X}$, any $K_{0}$-point $x \in X\left(K_{0}\right)$ extends in a unique way to an $R_{0}$-point $x \in \mathcal{X}\left(R_{0}\right)$; the special fiber of this point is the reduction of $x$ and is denoted $\operatorname{red}(x) \in \mathcal{X}\left(k_{0}\right)$. Let $D \subset X\left(K_{0}\right)$ be a finite set. A semistable model $\mathcal{X}$ of $X$ is a semistable $R_{0}$-model of $(X, D)$ provided that the points of $D$ reduce to distinct smooth points of $\mathcal{X}_{k_{0}}$. The model $\mathcal{X}$ is a stable $R_{0}$-model of $(X, D)$ provided that every rational (resp. genus-1) component of the normalization $\mathcal{X}_{k}$ contains at least three points (resp. one point) mapping to a singular point of $\mathcal{X}_{k}$ or to the reduction of a point of $D$.

If $K_{0}=K$ is complete and algebraically closed, we define a (strongly) semistable formal $R$-model of $X$ to be an integral, proper, admissible formal $R$-curve $\mathfrak{X}$ whose analytic generic fiber $\mathfrak{X}_{K}$ is equipped with an isomorphism to $X^{\text {an }}$ and whose special fiber $\mathfrak{X}_{k}$ is a (strongly) semistable curve. There is a natural map of sets red : $X^{\text {an }} \rightarrow \mathfrak{X}_{k}$, called the reduction, which extends the reduction map from the previous paragraph to the formal setting; it is surjective and anti-continuous in the sense that the inverse image of a Zariski-open subset of $\mathfrak{X}_{k}$ is a closed subset of $X^{\text {an }}$ (and vice versa). Using the reduction map, for a finite set $D \subset X(K)$, we define semistable and stable formal $R$-models of $(X, D)$ as above.

As we will be passing between formal and algebraic $R$-models of $K$-curves, it is worth stating the following lemma; see [12], Remark 4.2(2).

Lemma 5.1. Let $X$ be a smooth, proper, connected $K$-curve. The $\varpi$-adic completion functor defines an equivalence from the category of semistable $R$-models of $X$ to semistable formal $R$-models of $X$.

The inverse of the $\varpi$-adic completion functor of Lemma 5.1 will be called algebraization. 


\subsection{Descent to a general ground field}

We will use the following lemmas to descend the geometric theory of Section 4 to the valued field $K_{0}$. Fix an algebraic closure $\bar{K}_{0}$ of $K_{0}$ and a valuation val on $\bar{K}_{0}$ extending the given valuation on $K_{0}$. For any field $K_{1} \subset \bar{K}_{0}$, we consider $K_{1}$ as a valued field with respect to the restriction of val, and we write $R_{1}$ for the valuation ring of $K_{1}$. Let $K$ be the completion of $\bar{K}_{0}$ with respect to val. This field is algebraically closed by [20], Proposition 3.4.1/3.

Lemma 5.2. Let $X_{0}$ be a smooth, proper, geometrically connected curve over $K_{0}$, and let $X=X_{0} \otimes_{K_{0}}$ K. Let $K_{0}^{\text {sep }}$ be the separable closure of $K_{0}$ in $K$. Then, the image of $X_{0}\left(K_{0}^{\text {sep }}\right)$ under the natural inclusion

$$
X_{0}\left(K_{0}^{\text {sep }}\right) \subset X_{0}(K)=X(K) \subset X^{a n}
$$

is dense in $X^{a n}$.

Proof. Let $\widehat{K}_{0} \subset K$ denote the completion of $K_{0}$, and let $\left(\widehat{K}_{0}\right)^{\text {sep }} \subset K$ be its separable closure. By [20], Proposition 3.4.1/6), $\left(\widehat{K}_{0}\right)^{\text {sep }}$ is dense in $K$. Let $\widehat{K}_{1} \subset\left(\widehat{K}_{0}\right)^{\text {sep }}$ be a finite, separable extension of $\widehat{K}_{0}$. By Krasner's lemma (see $\S 3.4 .2$ of loc. cit.), there exists a finite, separable extension $K_{1} / K_{0}$ contained in $K_{0}$ which is dense in $\widehat{K}_{1}$. It follows that $K_{0}$ is dense in $\left(\widehat{K}_{0}\right)^{\text {sep }}$, so $K_{0}^{\text {sep }}$ is dense in $K$. Since $\mathbf{P}_{K}^{1}(K)$ is dense in $\mathbf{P}_{K}^{1, \text { an }}$ and the subspace topology on $\mathbf{P}_{K}^{1}(K) \subset \mathbf{P}_{K}^{1, \text { an }}$ coincides with the ultrametric topology, this proves the lemma for $X_{0}=\mathbf{P}_{K_{0}}^{1}$.

For general $X_{0}$, choose a finite, generically étale morphism $\varphi: X_{0} \rightarrow \mathbf{P}_{K_{0}}^{1}$. The map $\varphi$ is finite and flat, and hence open. It follows that if $U \subset X^{\text {an }}$ is a nonempty open set, then $\varphi(U)$ is a nonempty open subset of $\mathbf{P}_{K}^{1 \text {,an }}$. Let $x \in \mathbf{P}_{K_{0}}^{1}\left(K_{0}^{\text {sep }}\right)$ be a point contained in $\varphi(U)$. Then, $\varphi^{-1}(x) \subset X_{0}\left(K_{0}^{\text {sep }}\right)$ and $\varphi^{-1}(x) \cap U \neq \emptyset$.

Lemma 5.4. Let $X_{0}$ be a smooth, proper, geometrically connected curve over $K_{0}$, let $D \subset$ $X_{0}\left(K_{0}\right)$ be a finite subset, let $X=X_{0} \otimes_{K_{0}} K$, and let $\mathcal{X}$ be a semistable model of $(X, D)$. There exists a finite, separable extension $K_{1}$ of $K_{0}$ and a semistable model $\mathcal{X}_{1}$ of $X_{1}=X_{0} \otimes_{K_{0}} K_{1}$ with respect to $D$ such that $\mathcal{X}_{1} \otimes_{R_{1}} R \cong \mathcal{X}$.

Proof. Let $\mathfrak{X}$ be the $\varpi$-adic completion of $\mathcal{X}$. For $\bar{x} \in \mathfrak{X}_{k}(k)$, the subset red ${ }^{-1}(x) \subset X^{\text {an }}$ is open in $X^{\text {an }}$. By Lemma 5.2, there exists a point $x \in X_{0}\left(K_{0}^{\text {sep }}\right)$ with $\operatorname{red}(x)=\bar{x}$, so after passing to a finite, separable extension of $K_{0}$ if necessary, we may enlarge $D \subset X_{0}\left(K_{0}\right)$ to assume that $\mathcal{X}$ is a stable model of $(X, D)$. On the other hand, by the stable reduction theorem (i.e. the properness of the Deligne-Mumford stack $\overline{\mathcal{M}}_{g, n}$ parameterizing stable marked curves), there exists a stable model $\mathcal{X}_{0}$ of $\left(X_{0}, D_{0}\right)$ after potentially passing to a finite, separable extension of $K_{0}$. By uniqueness of stable models (i.e. the separateness of the Deligne-Mumford stack), we have $\mathcal{X}_{0} \otimes_{R_{0}} R \cong \mathcal{X}$.

Lemma 5.5. Let $X_{0}, X_{0}^{\prime}$ be smooth, proper, geometrically connected $K_{0}$-curves, and let $\varphi$ : $X_{0}^{\prime} \rightarrow X_{0}$ be a finite morphism. Let $\mathcal{X}_{0}$ (resp. $\left.\mathcal{X}_{0}^{\prime}\right)$ be a semistable model of $X_{0}$ (resp. $X_{0}^{\prime}$ ), let $X=X_{0} \otimes_{K_{0}} K$ and $\mathcal{X}=\mathcal{X}_{0} \otimes_{R_{0}} R$ (resp. $X^{\prime}=X_{0}^{\prime} \otimes_{K_{0}} K$ and $\mathcal{X}^{\prime}=\mathcal{X}_{0}^{\prime} \otimes_{R_{0}} R$ ), and suppose that $\varphi_{K}: X^{\prime} \rightarrow X$ extends to a morphism $\mathcal{X}^{\prime} \rightarrow \mathcal{X}$. Then, $\varphi$ extends to a morphism $\mathcal{X}_{0}^{\prime} \rightarrow \mathcal{X}_{0}$ in a unique way. 
Proof. The morphism $\varphi$ gives rise to a section $X_{0}^{\prime} \rightarrow X_{0} \times_{K_{0}} X_{0}^{\prime}$ of $\mathrm{pr}_{2}: X_{0} \times_{K_{0}} X_{0}^{\prime} \rightarrow X_{0}^{\prime}$. Let $\mathcal{Z}$ be the schematic closure of the image of $X_{0}^{\prime}$ in $\mathcal{X}_{0} \times_{R_{0}} \mathcal{X}_{0}^{\prime}$. It is enough to show that $\mathrm{pr}_{2}: \mathcal{Z} \rightarrow \mathcal{X}_{0}^{\prime}$ is an isomorphism. Since schematic closure respects flat base change, the schematic closure of the image of $X^{\prime}$ in $\mathcal{X} \times_{R} \mathcal{X}^{\prime}$ is equal to $\mathcal{Z} \otimes_{R_{0}} R$. By properness of $\mathcal{X}^{\prime}$, the image of $\mathcal{X}^{\prime}$ in $\mathcal{X} \times_{R} \mathcal{X}^{\prime}$ is then equal to $\mathcal{Z} \otimes_{R_{0}} R$, so $\mathrm{pr}_{2}: \mathcal{Z} \otimes_{R_{0}} R \rightarrow \mathcal{X}^{\prime}$ is an isomorphism. Since $R_{0} \rightarrow R$ is faithfully flat, this implies that $\mathrm{pr}_{2}: \mathcal{Z} \rightarrow \mathcal{X}_{0}^{\prime}$ is an isomorphism.

\subsection{The relation between semistable models and skeleta}

Let $X$ be a smooth, proper, connected $K$-curve. The following theorem is due to Berkovich and Bosch-Lütkebohmert; see [12], Theorem 4.6, for more precise references.

Theorem 5.7. Let $\mathfrak{X}$ be a semistable formal model of $X$, and let $\bar{x} \in \mathfrak{X}_{k}$ be a point. Then,

1. $\bar{x}$ is a generic point if and only if red ${ }^{-1}(\bar{x})$ consists of a single type-2 point of $X^{\text {an }}$.

2. $\bar{x}$ is a smooth closed point if and only if red $^{-1}(\bar{x})$ is an open ball.

3. $\bar{x}$ is an ordinary double point if and only if $\mathrm{red}^{-1}(\bar{x})$ is an open annulus.

It follows from Theorem 5.7 that if $\mathfrak{X}$ is a semistable formal model of $X$, then

$$
\left.V(\mathfrak{X})=\left\{\operatorname{red}^{-1} \bar{\zeta}\right): \zeta \in \mathfrak{X}_{k} \text { is a generic point }\right\}
$$

is a semistable vertex set of $X$ : indeed,

$$
X \backslash V(\mathfrak{X})=\coprod_{\substack{\bar{x} \in \mathfrak{X}_{k} \\ \text { singular }}} \operatorname{red}^{-1}(\bar{x}) \cup \coprod_{\substack{\bar{x} \in \mathfrak{X}_{k} \\ \text { smooth }}} \operatorname{red}^{-1}(\bar{x})
$$

is a disjoint union of open balls and finitely many open annuli. The following folklore theorem is proved in [12], Theorem 4.11.

Theorem 5.8. Let $X$ be a smooth, proper, connected algebraic $K$-curve, and let $D \subset X(K)$ be a finite set of closed points. The association $\mathfrak{X} \mapsto V(\mathfrak{X})$ sets up a bijection between the set of (strongly) semistable formal models of $(X, D)$ (up to isomorphism) and the set of (strongly) semistable vertex sets of $(X, D)$.

Let $\mathfrak{X}$ be a semistable formal model of $(X, D)$. One can construct the metrized complex of curves $\Sigma(X, V(\mathfrak{X}) \cup D)$ directly from $\mathfrak{X}$, as follows. Let $V$ be the set of irreducible components of $\mathfrak{X}_{k}$, regarded as a set of vertices of a graph. For $x \in V$, we will write $C_{x}$ to denote the corresponding component of $\mathfrak{X}_{k}$. To every double point $\bar{x} \in \mathfrak{X}_{k}$, we associate an edge $e$ connecting the irreducible components of $\mathfrak{X}_{k}$ containing $\bar{x}$ (this is a loop edge if there is only one such component); if $C_{x}$ is such a component, then we set $\operatorname{red}_{x}(e)=\bar{x}$. The completed local ring of $\mathfrak{X}$ at $\bar{x}$ is isomorphic to $R \llbracket s, t \rrbracket /(s t-\pi)$ for some $\pi \in \mathfrak{m}_{R} \backslash\{0\}$; we define the length of $e$ to be $\ell(e)=\operatorname{val}(\pi)$. We connect a point $x \in D$ to the vertex corresponding to the irreducible component of $\mathfrak{X}_{k}$ containing $\operatorname{red}(x)$. These data define a metrized complex of curves $\Sigma(\mathfrak{X}, D)$. By Equation 5.7.1, the graphs underlying 
$\Sigma(\mathfrak{X}, D)$ and $\Sigma(X, V(\mathfrak{X}) \cup D)$ are naturally isomorphic. The edge lengths coincide see for instance [12], Proposition 4.10 - and the residue curves of the two metrized complexes are naturally isomorphic by [14], Proposition 2.4.4. From this, it is straightforward to verify that $\Sigma(\mathfrak{X}, D)$ and $\Sigma(X, V(\mathfrak{X}) \cup D)$ are identified as metrized complexes of curves.

In particular, a semistable formal model $\mathfrak{X}$ of $(X, D)$ is stable if and only if $V(\mathfrak{X})$ is a stable vertex set of $(X, D)$.

As another consequence of Theorem 5.8, we obtain the following compatibility of skeleta and extension of scalars.

Proposition 5.10. Let $X$ be a smooth, proper, connected $K$-curve, let $K^{\prime}$ be a complete and algebraically closed valued field extension of $K$, and let $\pi: X_{K^{\prime}}^{a n} \rightarrow X_{K}^{a n}$ be the canonical map. If $\mathfrak{X}$ is a semistable formal model of $X$, then

1. $\pi$ maps $V\left(\mathfrak{X}_{R^{\prime}}\right)$ bijectively onto $V(\mathfrak{X})$, and

2. $\pi$ maps $\Sigma\left(X_{K^{\prime}}, V\left(\mathfrak{X}_{R^{\prime}}\right) \cup D\right)$ bijectively onto $\Sigma(X, V(\mathfrak{X}) \cup D)$, with

$$
\pi: \Sigma\left(X_{K^{\prime}}, V\left(\mathfrak{X}_{R^{\prime}}\right) \cup D\right) \stackrel{\sim}{\longrightarrow} \Sigma(X, V(\mathfrak{X}) \cup D)
$$

an isomorphism of augmented metric graphs.

Proof. The reduction map is compatible with extension of scalars, in that the following square commutes

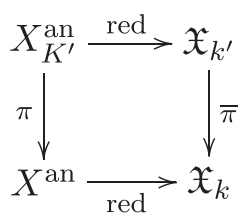

with $k^{\prime}$ the residue field of $K^{\prime}$. The first assertion is immediate because the extension of scalars morphism $\bar{\pi}: \mathfrak{X}_{k^{\prime}} \rightarrow \mathfrak{X}_{k}$ is a bijection on generic points. It is also a bijection on singular points, and for any node $\vec{x}^{\prime} \in \mathfrak{X}_{k^{\prime}}$ the open annulus $A^{\prime}=\operatorname{red}^{-1}\left(\vec{x}^{\prime}\right)$ is identified with the extension of scalars of $A=\operatorname{red}^{-1}\left(\bar{\pi}\left(\bar{x}^{\prime}\right)\right)$. It is easy to see that $\pi: A^{\prime} \rightarrow A$ takes the skeleton of $A^{\prime}$ isomorphically onto the skeleton of $A$.

\subsection{Extending morphisms to semistable models: analytic criteria}

Let $X$ be a smooth, proper, connected $K$-curve, and let $\mathfrak{X}$ be a semistable formal model of $X$. The inverse image topology on $X^{\text {an }}$ is the topology $\mathcal{T}(\mathfrak{X})$ whose open sets are the sets of the form $\operatorname{red}^{-1}\left(\mathfrak{U}_{k}\right)$, where $\mathfrak{U}_{k}$ is a Zariski-open subset of $\mathfrak{X}_{k}$. Note that any such set is closed in the natural topology on $X^{\text {an }}$. If $\mathfrak{U}_{k} \subset \mathfrak{X}_{k}$ is an affine open subset, then $\mathfrak{U}_{k}$ is the special fiber of a formal affine open $\mathfrak{U}=\operatorname{Spf}(\mathcal{A}) \subset \mathfrak{X}$, the set underlying the generic fiber $\mathfrak{U}_{K}=\mathscr{M}\left(\mathcal{A}_{K}\right) \subset X^{\text {an }}$ is equal to $\operatorname{red}^{-1}\left(\mathfrak{U}_{k}\right)$, and $\mathcal{A}$ is equal to the ring $\stackrel{\circ}{K}_{K}$ of power-bounded elements in the affinoid algebra $\mathcal{A}_{K}$. This essentially means that $\mathfrak{X}$ is a formal analytic variety in the sense of [18]; see [12], 
Remark 4.2(3), for an explanation. Therefore, $\mathfrak{X}$ is constructed by gluing the canonical models of the affinoid subdomains of $X^{\text {an }}$ which are open in the inverse image topology $\mathcal{T}(\mathfrak{X})$, along the canonical models of their intersections; here, the canonical model of an affinoid space $\mathscr{M}(\mathcal{B})$ is by definition $\operatorname{Spf}(\stackrel{\circ}{\mathcal{B}})$. The following general fact about formal analytic varieties follows from these observations and the functoriality of the reduction map.

Proposition 5.12. Let $X, X^{\prime}$ be smooth, proper, connected algebraic curves over $K$, let $\varphi$ : $X^{\prime} \rightarrow X$ be a finite morphism, and let $\mathfrak{X}$ and $\mathfrak{X}^{\prime}$ be semistable formal models of $X$ and $X^{\prime}$, respectively. Then, $\varphi$ extends to a morphism $\mathfrak{X}^{\prime} \rightarrow \mathfrak{X}$ if and only if $\varphi$ is continuous with respect to the inverse image topologies $\mathcal{T}(\mathfrak{X})$ and $\mathcal{T}\left(\mathfrak{X}^{\prime}\right)$, in which case there is exactly one morphism $\mathfrak{X}^{\prime} \rightarrow \mathfrak{X}$ extending $\varphi$.

Fix smooth, proper, connected algebraic $K$-curves $X, X^{\prime}$ and a finite morphism $\varphi: X^{\prime} \rightarrow$ $X$. The following theorem is well known to experts, although no proof appears in the published literature to the best of our knowledge (see however the book in preparation by Ducros ([25], Section 6.3), where part of this result is discussed).

Theorem 5.13. Let $\mathfrak{X}$ and $\mathfrak{X}^{\prime}$ be semistable formal models of $X$ and $X^{\prime}$, respectively. Then $\varphi: X^{\prime} \rightarrow X$ extends to a morphism $\mathfrak{X}^{\prime} \rightarrow \mathfrak{X}$ if and only if $\varphi^{-1}(V(\mathfrak{X})) \subset V\left(\mathfrak{X}^{\prime}\right)$, and $\mathfrak{X}^{\prime} \rightarrow \mathfrak{X}$ is finite if and only if $\varphi^{-1}(V(\mathfrak{X}))=V\left(\mathfrak{X}^{\prime}\right)$.

Remark 5.14. If $\mathfrak{X}$ and $\mathfrak{X}^{\prime}$ are semistable formal models of the punctured curves $(X, D)$ and $\left(X^{\prime}, D^{\prime}\right)$, respectively, and $\varphi: X^{\prime} \rightarrow X$ is a finite morphism with $\varphi^{-1}(D)=D^{\prime}$ which extends to a morphism $\mathfrak{X}^{\prime} \rightarrow \mathfrak{X}$, then since $\varphi^{-1}(V(\mathfrak{X})) \subset V\left(\mathfrak{X}^{\prime}\right)$ it follows from Remark 4.29 that there is a natural harmonic morphism of metric graphs $\Sigma\left(X^{\prime}, V\left(\mathfrak{X}^{\prime}\right) \cup\right.$ $\left.D^{\prime}\right) \rightarrow \Sigma(X, V(\mathfrak{X}) \cup D)$. The morphism $\mathfrak{X}^{\prime} \rightarrow \mathfrak{X}$ is finite if and only if the local degree of this harmonic morphism at every $v^{\prime} \in V\left(\mathfrak{X}^{\prime}\right)$ is positive.

Before giving a proof of Theorem 5.13, we mention the following consequences. Let $\mathfrak{X}_{1}$ and $\mathfrak{X}_{2}$ be semistable formal models of $X$. We say that $\mathfrak{X}_{1}$ dominates $\mathfrak{X}_{2}$ provided that there exists a (necessarily unique) morphism $\mathfrak{X}_{1} \rightarrow \mathfrak{X}_{2}$ inducing the identity map on analytic generic fibers. Taking $X=X^{\prime}$ in Theorem 5.13, we obtain the following corollary; this gives a different proof of the second part of ([12], Theorem 4.11).

Corollary 5.15. Let $\mathfrak{X}_{1}$ and $\mathfrak{X}_{2}$ be semistable formal models of $X$. Then, $\mathfrak{X}_{1}$ dominates $\mathfrak{X}_{2}$ if and only if $V\left(\mathfrak{X}_{1}\right) \supset V\left(\mathfrak{X}_{2}\right)$.

In conjunction with Proposition 5.10, we obtain the following corollary. If $K^{\prime}$ is a complete and algebraically closed field extension of $K$, we say that a semistable formal model $\mathfrak{X}^{\prime}$ of $X_{K^{\prime}}$ is defined over $R$ provided that it arises as the extension of scalars of a (necessarily unique) semistable formal model of $X$.

Corollary 5.16. Let $K^{\prime}$ be a complete and algebraically closed field extension of $K$, let $\mathfrak{X}^{\prime}$ be a semistable formal model of $X_{K^{\prime}}$, and suppose that there exists a semistable formal model $\mathfrak{X}$ of $X$ such that $\mathfrak{X}_{R^{\prime}}$ dominates $\mathfrak{X}^{\prime}$. Then, $\mathfrak{X}^{\prime}$ is defined over $R$. 
Proof. Let $\pi: X_{K^{\prime}}^{\text {an }} \rightarrow X^{\text {an }}$ be the canonical map. By Corollary 5.15, we have $V\left(\mathfrak{X}_{R^{\prime}}\right) \supset$ $V\left(\mathfrak{X}^{\prime}\right)$, and by Proposition 5.10 the map $\pi$ defines a bijection $V\left(\mathfrak{X}_{R^{\prime}}\right) \stackrel{\sim}{\longrightarrow} V(\mathfrak{X})$ and an isomorphism

$$
\Sigma\left(X_{K^{\prime}}, V\left(\mathfrak{X}_{R^{\prime}}\right)\right) \stackrel{\sim}{\longrightarrow} \Sigma(X, V(\mathfrak{X})) .
$$

Let $V=V(\mathfrak{X})$ and $V^{\prime}=\pi\left(V\left(\mathfrak{X}^{\prime}\right)\right)$. We claim that $V^{\prime}$ is a semistable vertex set. Granted this, letting $\mathfrak{Y}$ be the semistable formal model of $X$ associated to $V^{\prime}$, we have $V\left(\mathfrak{Y}_{R^{\prime}}\right)=$ $V\left(\mathfrak{X}^{\prime}\right)$ since $V\left(\mathfrak{Y}_{R^{\prime}}\right) \subset V\left(\mathfrak{X}_{R^{\prime}}\right)\left(\right.$ as $\mathfrak{X}_{R^{\prime}}$ dominates $\left.\mathfrak{Y}_{R^{\prime}}\right)$ and $\pi\left(V\left(\mathfrak{Y}_{R^{\prime}}\right)\right)=V(\mathfrak{Y})$, so $\mathfrak{Y}_{R^{\prime}}=\mathfrak{X}^{\prime}$.

It remains to prove the claim that $V^{\prime}$ is a semistable vertex set. The point is that the question of whether or not a subset of $V$ is a semistable vertex set is intrinsic to the augmented $\Lambda$-metric graph $\Sigma=\Sigma(X, V) \cong \Sigma\left(X_{K^{\prime}}, V\left(\mathfrak{X}_{R^{\prime}}\right)\right)$. We leave the details to the reader.

We will use the following lemmas in the proof of Theorem 5.13. Recall from (3.11.1) that if $V$ is a semistable vertex set of $X$, then a connected component $C$ of $X^{\text {an }} \backslash V$ is adjacent to a point $x \in V$ provided that the closure of $C$ in $X^{\text {an }}$ contains $x$.

Lemma 5.17. Let $\mathfrak{X}$ be a semistable formal model of $X$, let $V=V(\mathfrak{X})$, let $\mathfrak{U}_{k} \subset \mathfrak{X}_{k}$ be a subset, and let $U=\operatorname{red}^{-1}\left(\mathfrak{U}_{k}\right) \subset X^{a n}$. Then, $U$ is open in the topology $\mathcal{T}(\mathfrak{X})$ if and only if the following conditions hold:

1. $U$ is closed in the ordinary topology on $X^{\text {an }}$, and

2. for every $x \in U \cap V$, all but finitely many connected components of $X^{\text {an }} \backslash V$ which are adjacent to $x$ are contained in $U$.

Proof. Let $\bar{\zeta}$ be a generic point of $\mathfrak{X}_{k}$, and let $\bar{y} \in \mathfrak{X}_{k}$ be a closed point. Let $x \in V$ be the unique point of $X^{\text {an }}$ reducing to $\bar{\zeta}$, and let $B=\operatorname{red}^{-1}(\bar{y})$. By the anti-continuity of the reduction map, $\bar{y}$ is in the closure of $\{\bar{\zeta}\}$ if and only if $x$ is adjacent to $B$. The lemma follows easily from this and the fact that the connected components of $X^{\text {an }} \backslash V$ are exactly the inverse images of the closed points of $\mathfrak{X}_{k}$ under red.

Lemma 5.18. Let $V$ and $V^{\prime}$ be semistable vertex sets of $X$ and $X^{\prime}$, respectively, and suppose that $\varphi^{-1}(V) \subset V^{\prime}$. Let $C^{\prime}$ be a connected component of $X^{\prime a n} \backslash \varphi^{-1}(V)$. Then, $C^{\prime}$ has the following form:

1. If $C^{\prime}$ intersects $\Sigma\left(X^{\prime}, V^{\prime}\right)$, then $C^{\prime}=\tau^{-1}\left(\Sigma\left(X^{\prime}, V^{\prime}\right) \cap C^{\prime}\right)$, and

2. otherwise $C^{\prime}$ is an open ball connected component of $X^{\prime a n} \backslash V^{\prime}$.

Proof. Let $\Sigma^{\prime}=\Sigma\left(X^{\prime}, V^{\prime}\right)$. Suppose that there exists $y^{\prime} \in C^{\prime}$ such that $\tau\left(y^{\prime}\right) \notin C^{\prime}$. Let $B^{\prime}$ be the connected component of $X^{\prime \text { an }} \backslash \Sigma^{\prime}$ containing $y^{\prime}$, so $B^{\prime}$ is an open ball contained in $C^{\prime}$ and $\tau\left(y^{\prime}\right)$ is the end of $B^{\prime}$. It follows that $\tau\left(y^{\prime}\right)$ is contained in the closure of $C^{\prime}$ in $X^{\prime a n}$. Since $C^{\prime}$ is a connected component of $X^{\prime a n} \backslash \varphi^{-1}(V)$, its closure is contained in $C^{\prime} \cup \varphi^{-1}(V)$, so $\tau\left(y^{\prime}\right) \in \varphi^{-1}(V)$. Therefore, $B^{\prime}=\left(B^{\prime} \cup\left\{\tau\left(y^{\prime}\right)\right\}\right) \cap\left(X^{\prime a n} \backslash \varphi^{-1}(V)\right)$ is open and closed in $X^{\text {an }} \backslash \varphi^{-1}(V)$, so $B^{\prime}$ is a connected component of $X^{\prime \text { an }} \backslash \varphi^{-1}(V)$ and hence $B^{\prime}=C^{\prime}$.

Now, suppose that $\tau\left(C^{\prime}\right) \subset C^{\prime}$. Then, $\tau\left(C^{\prime}\right) \subset C^{\prime} \cap \Sigma^{\prime}$, so $C^{\prime} \subset \tau^{-1}\left(C^{\prime} \cap \Sigma^{\prime}\right)$. Let $y^{\prime} \in \tau^{-1}\left(C^{\prime} \cap \Sigma^{\prime}\right)$, and let $B^{\prime}$ be the connected component of $X^{\text {an }} \backslash \Sigma^{\prime}$ containing $y^{\prime}$, as 
above. Since $\bar{B}^{\prime}=B^{\prime} \cup\left\{\tau\left(y^{\prime}\right)\right\}$ is a connected subset of $X^{\prime a n} \backslash \varphi^{-1}(V)$ intersecting $C^{\prime}$, we have $\bar{B}^{\prime} \subset C^{\prime}$, so $y^{\prime} \in C^{\prime}$ and therefore $C^{\prime}=\tau^{-1}\left(C^{\prime} \cap \Sigma^{\prime}\right)$.

Proof. Let $V=V(\mathfrak{X})$, and $V^{\prime}=V\left(\mathfrak{X}^{\prime}\right)$. If there is an extension $\mathfrak{X}^{\prime} \rightarrow \mathfrak{X}$ of $\varphi$, then the square

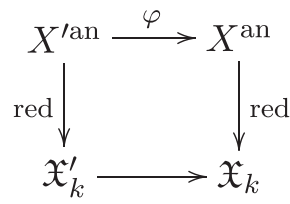

commutes. Let $x^{\prime} \in \varphi^{-1}(V)$, so $x=\varphi\left(x^{\prime}\right)$ reduces to a generic point $\bar{\zeta}$ of $\mathfrak{X}_{k}$. Since the reduction $\overline{\zeta^{\prime}}$ of $x^{\prime}$ maps to $\bar{\zeta}$, the point $\bar{\zeta}^{\prime}$ is generic, so $x^{\prime} \in V^{\prime}$. Therefore, $\varphi^{-1}(V) \subset V^{\prime}$. The morphism $\mathfrak{X}^{\prime} \rightarrow \mathfrak{X}$ is finite if and only if every generic point of $\mathfrak{X}_{k}^{\prime}$ maps to a generic point of $\mathfrak{X}_{k}$; as above, this is equivalent to $V^{\prime}=\varphi^{-1}(V)$.

It remains to prove that if $\varphi^{-1}(V) \subset V^{\prime}$, then $\varphi$ extends to a morphism $\mathfrak{X}^{\prime} \rightarrow \mathfrak{X}$. By Proposition 5.12, we must show that $\varphi$ is continuous with respect to the topologies $\mathcal{T}(\mathfrak{X})$ and $\mathcal{T}\left(\mathfrak{X}^{\prime}\right)$. Let $U \subset X^{\text {an }}$ be $\mathcal{T}(\mathfrak{X})$ open, and let $U^{\prime}=\varphi^{-1}(U)$. Clearly $U$ is closed, so $U^{\prime}$ is closed (with respect to the ordinary topologies). We must show that condition (2) of Lemma 5.17 holds for $U^{\prime}$. Let $x^{\prime} \in U^{\prime} \cap V^{\prime}$, and let $x=\varphi\left(x^{\prime}\right)$. If $x \notin V$, then let $C \subset U$ be the connected component of $X^{\text {an }} \backslash V$ containing $x$ and let $C^{\prime}$ be the connected component of $X^{\prime \text { an }} \backslash \varphi^{-1}(V)$ containing $x^{\prime}$. Then, $C^{\prime} \subset U^{\prime}$ because $\varphi\left(C^{\prime}\right) \subset C$, and $C^{\prime}$ contains every connected component of $X^{\prime a n} \backslash V^{\prime}$ adjacent to $x^{\prime}$ since $C^{\prime}$ is an open neighborhood of $x^{\prime}$.

Now suppose that $x \in V$. Any connected component of $X^{\prime a n} \backslash V^{\prime}$ which is adjacent to $x^{\prime}$ maps into a connected component of $X^{\text {an }} \backslash V$ which is adjacent to $x$. There are finitely many connected components $X^{\text {an }} \backslash V$ which are adjacent to $x$ and not contained in $U$ by Lemma 5.17. Let $C$ be such a component. Since $\varphi$ is finite, there are only finitely many connected components of $\varphi^{-1}(C)$; each of these is a connected component of $X^{\prime a n} \backslash$ $\varphi^{-1}(V)$. If $C^{\prime}$ is such a connected component, then either $C^{\prime}$ is an open ball connected component of $X^{\prime a n} \backslash V^{\prime}$ or $C^{\prime}$ intersects $\Sigma^{\prime}=\Sigma\left(X^{\prime}, V^{\prime}\right)$ by Lemma 5.18. There are finitely many connected components of $X^{\prime a n} \backslash V^{\prime}$ which intersect $\Sigma^{\prime}$ - these are just the open annulus connected components of $X^{\prime a n} \backslash V^{\prime}$ - so there are only finitely many connected components of $X^{\prime a n} \backslash V^{\prime}$ contained in $\varphi^{-1}(C)$. Therefore, all but finitely many connected components of $X^{\prime a n} \backslash V^{\prime}$ which are adjacent to $x^{\prime}$ map to connected components of $X^{\text {an }} \backslash V$ which are contained in $U$.

\subsection{Simultaneous semistable reduction theorems}

Recall that $K_{0}$ is a field equipped with a nontrivial non-Archimedean valuation val : $K_{0} \rightarrow$ $\mathbf{R} \cup\{\infty\}$. As above, we fix an algebraic closure $\bar{K}_{0}$ of $K_{0}$ and a valuation val on $\bar{K}_{0}$ extending the given valuation on $K_{0}$; for any field $K_{1} \subset \bar{K}_{0}$, we consider $K_{1}$ as a valued field with respect to the restriction of val, and we write $R_{1}$ for the valuation ring of $K_{1}$. In what follows, $X$ and $X^{\prime}$ are smooth, proper, geometrically connected $K_{0}$-curves and $\varphi: X^{\prime} \rightarrow X$ is a finite morphism.

Proposition 5.20. Let $\mathcal{X}$ be a semistable $R_{0}$-model of $X$. If there exists a semistable $R_{0}$-model $\mathcal{X}^{\prime}$ of $X^{\prime}$ such that $\varphi: X^{\prime} \rightarrow X$ extends to a finite morphism $\mathcal{X}^{\prime} \rightarrow \mathcal{X}$, then there is exactly one such model $\mathcal{X}^{\prime}$ up to (unique) isomorphism. 
Proof. Suppose first that $K=K_{0}$ is complete and algebraically closed. Let $\mathfrak{X}$ (resp. $\left.\mathfrak{X}^{\prime}\right)$ be the $\varpi$-adic completion of $\mathcal{X}$ (resp. $\mathcal{X}^{\prime}$ ). In this case, the proposition follows from Theorem 5.8 and the fact that if the morphism $\mathfrak{X}^{\prime} \rightarrow \mathfrak{X}$ of Theorem 5.13 is finite, then $V\left(\mathfrak{X}^{\prime}\right)$ is uniquely determined by $V(\mathfrak{X})$. The general case follows from this case after passing to the completion of the algebraic closure $K$ of $K_{0}$ : if $\mathcal{X}^{\prime}, \mathcal{X}^{\prime \prime}$ are two semistable models of $X^{\prime}$ such that $\varphi$ extends to finite morphisms $\mathcal{X}^{\prime} \rightarrow \mathcal{X}$ and $\mathcal{X}^{\prime \prime} \rightarrow \mathcal{X}$, then the isomorphism $\mathcal{X}_{R}^{\prime \prime} \stackrel{\sim}{\longrightarrow} \mathcal{X}_{R}^{\prime}$ descends to an isomorphism $\mathcal{X}^{\prime \prime} \stackrel{\sim}{\longrightarrow} \mathcal{X}^{\prime}$ by Lemma 5.5.

Let $\mathcal{X}_{1}, \mathcal{X}_{2}$ be semistable $R_{0}$-models of $X$. Recall that $\mathcal{X}_{1}$ dominates $\mathcal{X}_{2}$ if there exists a (necessarily unique) morphism $\mathcal{X}_{1} \rightarrow \mathcal{X}_{2}$ inducing the identity on $X$.

Proposition 5.21. Let $\mathcal{X}$ and $\mathcal{X}^{\prime}$ be semistable $R_{0}$-models of $X$ and $X^{\prime}$, respectively. Let $D^{\prime} \subset X^{\prime}\left(K_{0}\right)$ be a finite set of points. Then there exists a finite, separable extension $K_{1}$ of $K_{0}$ and a semistable $R_{1}$-model $\mathcal{X}^{\prime \prime}$ of $\left(X_{K_{1}}^{\prime}, D^{\prime}\right)$ such that:

1. $\mathcal{X}^{\prime \prime}$ dominates $\mathcal{X}_{R_{1}}^{\prime}$,

2. for any valued field extension $K_{1} \rightarrow K_{1}^{\prime}$, the morphism $\varphi_{K_{1}^{\prime}}: X_{K_{1}^{\prime}}^{\prime} \rightarrow X_{K_{1}^{\prime}}$ extends to a morphism $\mathcal{X}_{R_{1}^{\prime}}^{\prime \prime} \rightarrow \mathcal{X}_{R_{1}^{\prime}}$, where $R_{1}^{\prime}$ is the ring of integers in $K_{1}^{\prime}$, and

3. any other semistable $R_{1}$-model of $\left(X_{K_{1}}^{\prime}, D^{\prime}\right)$ satisfying the above two properties dominates $\mathcal{X}^{\prime \prime}$.

Proof. Suppose first that $K_{0}=K$ is complete and algebraically closed. Let $\mathfrak{X}$ and $\mathfrak{X}^{\prime}$ be the $\varpi$-adic completions of $\mathcal{X}$ and $\mathcal{X}^{\prime}$, respectively. Let $V=V(\mathfrak{X})$ and $V^{\prime}=V\left(\mathfrak{X}^{\prime}\right)$. By Lemma 3.15, there is a minimal semistable vertex set $V^{\prime \prime}$ of $\left(X^{\prime}, D^{\prime}\right)$ which contains $\varphi^{-1}(V) \cup V^{\prime}$. Let $\mathfrak{X}^{\prime \prime}$ be the semistable formal model of $X^{\prime}$ corresponding to $V^{\prime \prime}$. Then, $\mathfrak{X}^{\prime \prime}$ dominates $\mathfrak{X}^{\prime}$ by Corollary 5.15 and $\varphi$ extends to a morphism $\mathfrak{X}^{\prime \prime} \rightarrow \mathfrak{X}$ by Theorem 5.13. Part 3 follows from Corollary 5.15 and the minimality of $V^{\prime \prime}$. Taking $\mathcal{X}^{\prime \prime}$ to be the algebraization of $\mathfrak{X}^{\prime \prime}$ yields (1) to (3) in this case.

For a general valued field $K_{0}$, suppose that $K$ is the completion of $\bar{K}_{0}$. By Lemma 5.4, the model of $X_{K}^{\prime}$ constructed above descends to a model $\mathcal{X}^{\prime \prime}$ defined over the ring of integers of a finite, separable extension $K_{1}$ of $K_{0}$. Properties (1)-(3) follow from Lemma 5.5 and the corresponding properties of $\mathcal{X}_{R}^{\prime \prime}$.

Now, we address the behavior of this construction with respect to base change. Using Lemma 5.5, we immediately reduce to the case of an extension $K \rightarrow K^{\prime}$ of complete and algebraically closed valued fields. Let $R^{\prime}$ be the ring of integers of $K^{\prime}$. Let $\mathcal{X}^{\prime \prime}$ (resp. $\mathcal{X}^{\prime \prime \prime}$ ) be the minimal $R$-model of $X^{\prime}$ dominating $\mathcal{X}^{\prime}$ (resp. $R^{\prime}$-model of $X_{K^{\prime}}^{\prime}$ dominating $\mathcal{X}_{R^{\prime}}^{\prime}$ ) mapping to $\mathcal{X}$ (resp. $\mathcal{X}_{R^{\prime}}$ ). By (3) as applied to $\mathcal{X}^{\prime \prime \prime}$, we have that $\mathcal{X}_{R^{\prime}}^{\prime \prime}$ dominates $\mathcal{X}^{\prime \prime \prime}$. By Corollary 5.16, $\mathcal{X}^{\prime \prime \prime}$ is defined over $R$, so by (3) as applied to $\mathcal{X}^{\prime \prime}$, we have $\mathcal{X}_{R^{\prime}}^{\prime \prime}=\mathcal{X}^{\prime \prime \prime}$.

Theorem 5.22 (Liu). Let $\mathcal{X}$ (resp. $\left.\mathcal{X}^{\prime}\right)$ be a semistable $R_{0}$-model of $X$ (resp. $\left.X^{\prime}\right)$. Let $D \subset$ $X\left(K_{0}\right)$ and $D^{\prime} \subset X^{\prime}\left(K_{0}\right)$ be finite sets, and suppose that $\varphi\left(D^{\prime}\right) \subset D$. Then, there exists a finite, separable extension $K_{1}$ of $K_{0}$ and semistable $R_{1}$-models $\mathcal{X}_{1}, \mathcal{X}_{1}^{\prime}$ of $\left(X_{K_{1}}, D\right),\left(X_{K_{1}}^{\prime}, D^{\prime}\right)$, respectively, such that

1. $\mathcal{X}_{1}$ dominates $\mathcal{X}_{R_{1}}$ and $\mathcal{X}_{1}^{\prime}$ dominates $\mathcal{X}_{R_{1}}^{\prime}$,

2. the morphism $\varphi_{K_{1}}: X_{K_{1}}^{\prime} \rightarrow X_{K_{1}}$ extends to a finite morphism $\mathcal{X}_{1}^{\prime} \rightarrow \mathcal{X}_{1}$, and 
3. if $\mathcal{X}_{2}, \mathcal{X}_{2}^{\prime}$ are semistable formal models of $\left(X_{K_{1}}, D\right),\left(X_{K_{1}}^{\prime}, D^{\prime}\right)$, respectively, satisfying (1) and (2) above, then $\mathcal{X}_{2}$ dominates $\mathcal{X}_{1}$ and $\mathcal{X}_{2}^{\prime}$ dominates $\mathcal{X}_{1}^{\prime}$.

Moreover, the formation of $\mathcal{X}_{1}^{\prime} \rightarrow \mathcal{X}_{1}$ commutes with arbitrary valued field extensions $K_{1} \rightarrow K_{1}^{\prime}$.

The morphism $\mathcal{X}_{1}^{\prime} \rightarrow \mathcal{X}_{1}$ is called the stable marked hull of $\mathcal{X}^{\prime} \rightarrow \mathcal{X}$ in [34].

Proof. First, assume that $K_{0}=K$ is complete and algebraically closed; let $\mathfrak{X}, \mathfrak{X}^{\prime}$ be the $\varpi$-adic completions of $\mathcal{X}, \mathcal{X}^{\prime}$, respectively. Let $V=V(\mathfrak{X})$ and $V^{\prime}=V\left(\mathfrak{X}^{\prime}\right)$. By Theorem 5.8, Theorem 5.13, and Corollary 5.15, we may equivalently formulate the existence and uniqueness of $\mathfrak{X}_{1}^{\prime} \rightarrow \mathfrak{X}_{1}$ in terms of semistable vertex sets, as follows. We must prove that there exists a semistable vertex set $V_{1}$ of $(X, D)$ such that

1. $V_{1}$ contains $V \cup \varphi\left(V^{\prime}\right)$,

2. $\varphi^{-1}\left(V_{1}\right)$ is a semistable vertex set of $\left(X^{\prime}, D^{\prime}\right)$, and

3. $V_{1}$ is minimal in the sense that if $V_{2}$ is another semistable vertex set of $(X, D)$ satisfying (1) and (2) above, then $V_{2} \supset V_{1}$.

First, we will prove the existence of $V_{1}$ satisfying (1) and (2). By Lemma 3.15, we may enlarge $V$ to assume that $V$ is a semistable vertex set of $(X, D)$. Let $\Sigma=\Sigma(X, V \cup D)$ and $\Sigma^{\prime}=\Sigma\left(X^{\prime}, V^{\prime}\right)$. By Corollary 4.18, there exists a skeleton $\Sigma_{1}$ of $(X, D)$ such that $\Sigma_{1} \supset \Sigma \cup \varphi\left(\Sigma^{\prime}\right)$ and such that $\Sigma_{1}^{\prime}=\varphi^{-1}\left(\Sigma_{1}\right)$ is a skeleton of $\left(X^{\prime}, \varphi^{-1}(D)\right)$. Let $V_{1}^{\prime}$ be a vertex set for $\Sigma_{1}^{\prime}$. Since any finite subset of type-2 points of $\Sigma_{1}^{\prime}$ which contains $V_{1}^{\prime}$ is again a vertex set for $\Sigma_{1}^{\prime}$ by Proposition 3.12(4), we may and do assume that $V^{\prime} \subset V_{1}^{\prime}$. Let $V_{1} \subset \Sigma_{1}$ be the union of a vertex set for $\Sigma_{1}$ with $V \cup \varphi\left(V_{1}^{\prime}\right)$. Then, $V_{1}$ is a semistable vertex set of $(X, D)$, and $\varphi^{-1}\left(V_{1}\right) \subset \Sigma_{1}^{\prime}$ is a finite set of type-2 points containing $V_{1}^{\prime}$, thus is a semistable vertex set of $\left(X^{\prime}, \varphi^{-1}(D)\right)$ (hence of $\left(X^{\prime}, D^{\prime}\right)$ as well).

To prove that there exists a minimal such $V_{1}$, we make the following recursive construction. Let $V(0)=V$, let $V^{\prime}(0)=V^{\prime}$, and for each $n \geq 1$ let $V(n)$ be the minimal semistable vertex set of $(X, D)$ containing $V(n-1) \cup \varphi\left(V^{\prime}(n-1)\right)$, and let $V^{\prime}(n)$ be the minimal semistable vertex set of $\left(X^{\prime}, D^{\prime}\right)$ containing $\varphi^{-1}(V(n))$. These sets exist by Lemma 3.15. By induction, it is clear that if $V_{2}$ is any semistable vertex set of $(X, D)$ satisfying (1) and (2) above, then $V(n) \subset V_{2}$ for each $n$. Since $V_{2}$ is a finite set, for some $n$ we have $V(n)=V(n+1)$, which is to say that $\varphi\left(V^{\prime}(n)\right) \subset V(n)$; since $V^{\prime}(n) \supset \varphi^{-1}(V(n))$, we have that $V^{\prime}(n)=\varphi^{-1}(V(n))$ is a semistable vertex set of $\left(X^{\prime}, D^{\prime}\right)$. Then, $V_{1}=V(n)$ is the minimal semistable vertex set satisfying (1) and (2) above.

The case of a general ground field reduces to the geometric case handled above exactly as in the proof of Proposition 5.21, as does the statement about the behavior of the stable marked hull with respect to valued field extensions.

Remark 5.23 (The skeletal viewpoint on Liu's theorem). The statement of Theorem 5.22 is strongly analogous to Corollary 4.18, which is indeed the main ingredient in the proof. The difference is that whereas finite morphisms of semistable models correspond to pairs $V, V^{\prime}$ of semistable vertex sets such that $\varphi^{-1}(V)=V^{\prime}$, for finite morphisms of triangulated punctured curves one requires in addition that $\varphi^{-1}(\Sigma)=\Sigma^{\prime}$. 
The former condition (of Liu's theorem) does not imply the latter: for instance, let $X^{\prime}$ be the Tate curve $y^{2}=x^{3}+x^{2}+\varpi$, let $X=\mathbf{P}^{1}$, and let $\varphi: X^{\prime} \rightarrow X$ be the cover $(x, y) \mapsto x$. This extends to a finite morphism of semistable models given by the same equations; however, the associated skeleton of $X^{\prime}$ (resp. of $\mathbf{P}^{1}$ ) is a circle (resp. a point), so $\varphi^{-1}(\Sigma) \subsetneq \Sigma^{\prime}$.

In general, if $\varphi^{-1}(V)=V^{\prime}$ and $\varphi^{-1}(D)=D^{\prime}$, then by Remark 4.19 the image of the skeleton $\Sigma\left(X^{\prime}, V^{\prime} \cup D^{\prime}\right)$ is equal to $\Sigma(X, V \cup D)$ union a finite number of geodesic segments $T_{1}, \ldots, T_{r}$, and by Theorem 4.13 the saturation $\varphi^{-1}\left(\varphi\left(\Sigma\left(X^{\prime}, V^{\prime} \cup D^{\prime}\right)\right)\right.$ is a skeleton of $X^{\prime}$.

Theorem 5.25 below follows the same philosophy in deriving a simultaneous semistable reduction theorem of Liu-Lorenzini from Proposition 4.12.

Remark 5.24. Liu in fact works over an arbitrary Dedekind scheme (a connected Noetherian regular scheme of dimension 1), which includes discrete valuation rings but not more general valuation rings. In his statement of Theorem 5.22, the given models $\mathcal{X}, \mathcal{X}^{\prime}$ are allowed to be any integral, projective $\operatorname{Spec}\left(R_{0}\right)$ schemes with generic fibers $X$ and $X^{\prime}$, respectively. Although we restrict to semistable models $\mathcal{X}, \mathcal{X}^{\prime}$ in this paper, using a more general notion of a triangulation our methods can be extended to treat relatively normal models $\mathcal{X}, \mathcal{X}^{\prime}$.

The following simultaneous stable reduction theorem can be found in [32]. Theorem 5.25 is to Proposition 4.12 as Theorem 5.22 is to Corollary 4.18

Theorem 5.25 (Liu-Lorenzini). Suppose that $(X, D)$ and $\left(X^{\prime}, D^{\prime}\right)$ are both stable and that $\varphi^{-1}(D)=D^{\prime}$. Assume that $(X, D)$ and $\left(X^{\prime}, D^{\prime}\right)$ admit stable models $\mathcal{X}$ and $\mathcal{X}^{\prime}$, respectively, defined over $R_{0}$. Then, $\varphi: X^{\prime} \rightarrow X$ extends to a (not necessarily finite) morphism $\mathcal{X}^{\prime} \rightarrow \mathcal{X}$.

Proof. By Lemma 5.5, we may assume that $K_{0}=K$ is complete and algebraically closed. Let $V$ (resp. $\left.V^{\prime}\right)$ be the minimal semistable vertex set of $(X, D)\left(\right.$ resp. $\left.\left(X^{\prime}, D^{\prime}\right)\right)$. By Theorem 5.13, we must show that $\varphi^{-1}(V) \subset V^{\prime}$. Let $\Sigma=\Sigma(X, V)$ and $\Sigma^{\prime}=\Sigma\left(X^{\prime}, V^{\prime}\right)$. Let $V_{1}$ be a vertex set for $\Sigma$ with respect to which $\Sigma$ has no loop edges. Recall that

$$
V=\left\{x \in V_{1}: g(x) \geq 1 \text { or } x \text { has valency at least } 3\right\}
$$

by Proposition 3.18(2). Let $V_{1}^{\prime}=\varphi^{-1}\left(V_{1}\right) \cup V^{\prime}$; this is a vertex set for $\Sigma^{\prime}$ since $\varphi^{-1}(\Sigma) \subset$ $\Sigma^{\prime}$ by Proposition 4.12(2). If $x \in V$ has genus at least 1 , then any $x^{\prime} \in \varphi^{-1}(x)$ has genus at least 1; so, $\varphi^{-1}(x) \subset V^{\prime}$. Let $x \in V$ have genus 0 , so $x$ has valency at least 3 in $\Sigma$. Let $x^{\prime} \in \varphi^{-1}(x) \subset \Sigma^{\prime}$, and let $U^{\prime}$ be the set containing $x^{\prime}$ and all of the connected components of $X^{\prime \text { an }} \backslash V_{1}^{\prime}$ adjacent to $x^{\prime}$. Then, $U^{\prime}$ is an open neighborhood of $x^{\prime}$, so $\varphi\left(U^{\prime}\right)$ is an open neighborhood of $x$ by [14], Lemma 3.2.4. Let $A$ be an open annulus connected component of $X^{\text {an }} \backslash V_{1}$ adjacent to $x$. Any open neighborhood of $x$ intersects $A$, so there exists $y^{\prime} \in$ $U^{\prime}$ with $\varphi\left(y^{\prime}\right) \in A$. Let $A^{\prime}$ be the connected component of $U^{\prime} \backslash\left\{x^{\prime}\right\}$ containing $y^{\prime}$, so $\varphi\left(A^{\prime}\right) \subset A$. Since $x^{\prime}$ is an end of $A^{\prime}$ and $\varphi\left(x^{\prime}\right)$ is an end of $A$, Lemma 4.3 implies that $A^{\prime}$ is an open annulus. Since $x$ has valency at least 3 in $\Sigma$ (and since $\Sigma$ has no loop edges), there are at least 3 distinct open annulus connected components of $X^{\text {an }} \backslash V_{1}$ adjacent to 
$x$. By the above, the same is true of $x^{\prime}$, so $x^{\prime}$ has valency at least 3 in $\Sigma^{\prime}$, so $x^{\prime} \in V^{\prime}$, as desired.

\section{A local lifting theorem}

We now begin with the classification of lifts of harmonic morphisms of metrized complexes of curves to finite morphisms of algebraic curves. We begin by working in the neighborhood of a vertex of a metrized complex.

Let $X$ be a smooth, proper, connected curve over $K$, and let $x \in X^{\text {an }}$ be a type-2 point. Let $V \subset X^{\text {an }}$ be a strongly semistable vertex set of $X$ containing $x$, let $\Sigma=\Sigma(X, V)$, and let $\tau: X^{\text {an }} \rightarrow \Sigma$ be the retraction. Let $e_{1}, \ldots, e_{r}$ be the edges of $\Sigma$ adjacent to $x$ and let $\Sigma_{0}=\{x\} \cup e_{1}^{\circ} \cup \cdots \cup e_{r}^{\circ}$, where for a (closed) edge $e$ we let $e^{\circ}$ denote the corresponding open edge, i.e. the edge without its endpoints. Then, $\Sigma_{0}$ is an open neighborhood of $x$ in $\Sigma$ and $\tau^{-1}\left(\Sigma_{0}\right)$ is an open neighborhood of $x$ in $X^{\text {an }}$. Following [12], Definition 4.28, and [15], we define a simple neighborhood of $x$ to be an open neighborhood of this form (for some choice of $V$ ). The connected components of $\tau^{-1}\left(\Sigma_{0}\right) \backslash\{x\}$ are open balls and the open annuli $\tau^{-1}\left(e_{1}^{\circ}\right), \ldots, \tau^{-1}\left(e_{r}^{\circ}\right)$.

Definition 6.2. A star-shaped curve is a pointed $K$-analytic space $(Y, y)$ which is isomorphic to $(U, x)$ where $x$ is a type-2 point in the analytification of a smooth, proper, connected curve over $K$, and $U$ is a simple neighborhood of $x$. The point $y$ is called the central vertex of $Y$.

Let $(Y, y)$ be a star-shaped curve, so $Y \backslash\{y\}$ is a disjoint union of open balls and finitely many open annuli $A_{1}, \ldots, A_{r}$. The skeleton of $Y$ is defined to be the set $\Sigma(Y,\{y\})=\{y\} \cup$ $\bigcup_{i=1}^{r} \Sigma\left(A_{i}\right)$. A compatible divisor in $Y$ is a finite set $D \subset Y(K)$ whose points are contained in distinct open ball connected components of $Y \backslash\{y\}$, so the connected components of $Y \backslash(\{y\} \cup D)$ are open balls, the open annuli $A_{1}, \ldots, A_{r}$, and (finitely many) open balls $B_{1}, \ldots, B_{s}$ punctured at a point of $D$. The data $(Y, y, D)$ of a star-shaped curve along with a compatible divisor is called a punctured star-shaped curve. The skeleton of $(Y, y, D)$ is the set

$$
\Sigma(Y,\{y\} \cup D)=\{y\} \cup D \cup \bigcup_{i=1}^{r} \Sigma\left(A_{i}\right) \cup \bigcup_{j=1}^{s} \Sigma\left(B_{j}\right) .
$$

Fix a compatible divisor $D$ in $Y$, and let $\Sigma_{0}=\Sigma(Y,\{y\} \cup D)$. There is a canonical continuous retraction map $\tau: Y \rightarrow \Sigma_{0}$ defined exactly as for skeleta of algebraic curves (3.11.1). The connected components of $\Sigma_{0} \backslash\{y\}$ are called the edges of $\Sigma_{0}$; an edge is called infinite if it contains a point of $D$ and finite otherwise.

If $Y$ is the simple neighborhood $\tau^{-1}\left(\Sigma_{0}\right)$ of $x \in X^{\text {an }}$ as above, then $\Sigma(Y,\{x\})=$ $\Sigma_{0}=\Sigma \cap Y$. A finite set $D \subset Y(K)$ is compatible with $Y$ if and only if $V$ is a semistable vertex set for $(X, D)$, in which case $\Sigma(Y,\{x\} \cup D)=Y \cap \Sigma(X, V \cup D)$. The retraction $\tau: Y \rightarrow \Sigma(Y,\{x\} \cup D)$ is the restriction of the canonical retraction $\tau: X^{\text {an }} \rightarrow \Sigma(X, V \cup D)$.

Let $(Y, y)$ be a star-shaped curve. Then, $Y$ is proper as a $K$-analytic space if and only if all connected components of $Y \backslash\{y\}$ are open balls, i.e. if and only if $\Sigma(Y,\{y\})=$ $\{y\}$. If $Y$ is proper, then there is a smooth, proper, connected curve $X$ over $K$ and an isomorphism $f: Y \stackrel{\sim}{\longrightarrow} X^{\text {an }}$. Let $x=f(y)$. Then, $\{x\}$ is a semistable vertex 
set of $X$, so by Theorem 5.8 there is a unique smooth formal model $\mathfrak{X}$ of $X^{\text {an }}$ such that $x$ reduces to the generic point of $\mathfrak{X}_{k}$. Let $D \subset Y(K)$ be a finite set. Then, $D$ is compatible with $Y$ if and only if the points of $f(D)$ reduce to distinct closed points of $\mathfrak{X}_{k}$.

Conversely, let $\mathfrak{X}$ be a smooth, proper, connected formal curve over $\operatorname{Spf}(R)$. If $x \in X^{\text {an }}$ is the point reducing to the generic point of $\mathfrak{X}_{k}$, then $\left(\mathfrak{X}_{K}, x\right)$ is a proper star-shaped curve.

Proposition 6.5. Let $(Y, y)$ be a star-shaped curve. Then, $(Y, y)$ is isomorphic to $(U, x)$ where $x$ is the central vertex of a proper star-shaped curve $X$ and $U$ is a simple neighborhood of $x$.

Proof. Let $A_{1}, \ldots, A_{r}$ be the open annulus connected components of $Y \backslash\{y\}$. Choose isomorphisms $f_{i}: A_{i} \stackrel{\sim}{\longrightarrow} \mathbf{S}\left(a_{i}\right)_{+}$with standard open annuli such that $f_{i}(x)$ approaches the Gauss point of $\mathbf{B}(1)$ as $x$ approaches $y$. Let $X$ be the curve obtained from $Y$ by gluing an open ball $\mathbf{B}(1)_{+}$onto each $A_{i}$ via the inclusions $f_{i}: A_{i} \stackrel{\sim}{\longrightarrow} \mathbf{S}\left(a_{i}\right)_{+} \subset$ $\mathbf{B}(1)_{+}$. Then, $X$ is a smooth, proper, connected $K$-analytic curve, and it is clear from the construction that $(X, y)$ is star shaped and that $Y$ is a simple neighborhood of $y$ in $X$.

A proper star-shaped curve $X$ and an inclusion $i: Y \stackrel{\sim}{\longrightarrow} U \subset X$ as in Proposition 6.5 is called a compactification of $Y$ (as a star-shaped curve). Note that $X \backslash i(Y)$ is a disjoint union of finitely many closed balls, one for each open annulus connected component of $Y \backslash\{y\}$. Note also that, by construction, the genus of the algebraization of $X$ is equal to $g(y)$.

Let $(Y, y)$ be a star-shaped curve. The smooth, proper, connected $k$-curve $C_{y}$ with function field $\widetilde{\mathscr{H}}(y)$ is called the residue curve of $Y$. The tangent vectors in $T_{y}$ are naturally in bijective correspondence with the connected components of $Y \backslash\{y\}$. We define a reduction map red : $Y \rightarrow C_{y}$ by sending $y$ to the generic point of $C_{y}$ and sending every point in a connected component $B$ of $Y \backslash\{y\}$ to the closed point of $C_{y}$ corresponding to the tangent vector determined by $B$. This sets up a one-to-one correspondence between the connected components of $Y \backslash\{y\}$ and the closed points of $C_{y}$.

\section{Remark 6.8.}

1. When $Y$ is proper, so $Y \cong \mathfrak{X}_{K}$ for a smooth, proper, connected formal curve over $\operatorname{Spf}(R)$; then, $C_{y} \cong \mathfrak{X}_{k}$ and the reduction map $Y \rightarrow C_{y}$ coincides with the canonical reduction map $\mathfrak{X}_{K} \rightarrow \mathfrak{X}_{k}$.

2. Let $D \subset Y(K)$ be a compatible divisor, and let $\Sigma_{0}=\Sigma(Y,\{y\} \cup D)$. Every edge $e$ of $\Sigma_{0}$ is contained in a unique connected component of $Y \backslash\{y\}$, and $\operatorname{red}(e) \in C_{y}(k)$ is the closed point corresponding to the tangent direction represented by $e$.

\subsection{Tame coverings}

We now study a class of morphisms of star-shaped curves analogous to (4.30). We begin with the following technical result. 
Lemma 6.10. Let $A, A^{\prime}$ be open annuli or punctured open balls, and let $\varphi: A^{\prime} \rightarrow A$ be a finite morphism of degree $\delta$. Suppose that $\delta$ is prime to $\operatorname{char}(k)$ if $\operatorname{char}(k)>0$. Fix an isomorphism $A \cong \mathbf{S}(a)_{+}$(where we allow $a=0$ ).

1. There is an isomorphism $A^{\prime} \cong \mathbf{S}\left(a^{\prime}\right)+$ such that the composition

$$
\mathbf{S}\left(a^{\prime}\right)_{+} \cong A^{\prime} \stackrel{\varphi}{\longrightarrow} A \cong \mathbf{S}(a)_{+}
$$

is $t \mapsto t^{\delta}$.

2. There is an isomorphism $A^{\prime} \cong \mathbf{S}\left(a^{\prime}\right)+$ such that Equation 6.10.1 extends to a morphism $\psi: \mathbf{B}(1)_{+} \rightarrow \mathbf{B}(1)_{+}$with $\psi^{-1}(0)=0$.

3. If Equation 6.10.1 extends to a morphism $\psi: \mathbf{B}(1)_{+} \rightarrow \mathbf{B}(1)_{+}$for a given isomorphism $A^{\prime} \cong \mathbf{S}\left(a^{\prime}\right)_{+}$, then the extension is unique.

Proof. Let $u$ be a parameter on $A^{\prime}$, i.e. an isomorphism $u: A^{\prime} \stackrel{\sim}{\longrightarrow} \mathbf{S}\left(a^{\prime}\right)_{+} \subset \mathbf{B}(1)_{+}$ with a standard open annulus. By Lemma 4.2, $\varphi$ restricts to an affine map on skeleta $\Sigma\left(A^{\prime}\right) \rightarrow \Sigma(A)$ with degree $\delta$. If $B=\mathbf{S}(b, c)$ is a closed sub-annulus of $\mathbf{S}\left(a^{\prime}\right)_{+}$, then by [44], Lemme 2.2.1, after potentially replacing $u$ by $u^{-1}, \varphi^{*}(t)$ has the form $\alpha u^{\delta}(1+g(u))$ on $B$, where $\alpha \in R^{\times}$and $|g|_{\text {sup }}<1$. Since $\delta$ is not divisible by $\operatorname{char}(k)$ if $\operatorname{char}(k)>0$, the Taylor expansion for $\sqrt[\delta]{1+g}$ has coefficients contained in $R$, hence converges to a $\delta$ th root of $1+g(u)$ on $A$. Choosing a $\delta$ th root of $\alpha$ as well and letting $u_{B}=u \sqrt[\delta]{\alpha} \sqrt[\delta]{1+g}$, we have that $u_{B}$ is a parameter on $B$ such that $\varphi^{*}(t)=u_{B}^{\delta}$. Hence, for each such $B$, there are exactly $\delta$ choices of a parameter $u_{B}$ on $B$ such that $\varphi^{*}(t)=u_{B}^{\delta}$; choosing a compatible set of such parameters for all $B$ yields a parameter on $A^{\prime}$ satisfying (1).

Part (2) follows immediately from (1). A morphism from a $K$-analytic space $X$ to $\mathbf{B}(1)_{+}$ is given by a unique analytic function $f$ on $X$ such that $|f(x)|<1$ for all $x \in X$, so (3) follows from the fact that the restriction homomorphism $\mathrm{O}\left(\mathbf{B}(1)_{+}\right) \rightarrow \mathrm{O}\left(\mathbf{S}\left(a^{\prime}\right)_{+}\right)$is injective.

Let $A$ be an open annulus. Let $\delta$ be a positive integer and assume that $\delta$ is prime to the characteristic of $k$ if $\operatorname{char}(k)>0$. Let $A^{\prime}$ be an open annulus, and let $\varphi: A^{\prime} \rightarrow A$ be a finite morphism of degree $\delta$. By Lemma 6.10, the group $\operatorname{Aut}_{A}\left(A^{\prime}\right)$ is isomorphic a to $\mathbf{Z} / \delta \mathbf{Z}$. Moreover, if $A^{\prime \prime}$ is another open annulus and $\varphi^{\prime}: A^{\prime \prime} \rightarrow A$ is a finite morphism of degree $\delta$, then there exists an $A$-isomorphism $\psi: A^{\prime} \stackrel{\sim}{\longrightarrow} A^{\prime \prime}$; the induced isomorphism $\operatorname{Aut}_{A}\left(A^{\prime}\right) \stackrel{\sim}{\longrightarrow} \operatorname{Aut}_{A}\left(A^{\prime \prime}\right)$ is independent of the choice of $\psi$ since both groups are abelian. Therefore, the group $\operatorname{Aut}_{A}(\delta):=\operatorname{Aut}_{A}\left(A^{\prime}\right) \cong \mathbf{Z} / \delta \mathbf{Z}$ is canonically determined by $A$ and $\delta$.

Definition 6.12. Let $(Y, y, D)$ be a punctured star-shaped curve with skeleton $\Sigma_{0}=$ $\Sigma(Y,\{y\} \cup D)$, and let $C_{y}$ be the residue curve of $Y$. A tame covering of $(Y, y, D)$ consists of a punctured star-shaped curve $\left(Y^{\prime}, y^{\prime}, D^{\prime}\right)$ and a finite morphism $\varphi: Y^{\prime} \rightarrow Y$ satisfying the following properties:

1. $\varphi^{-1}(y)=\left\{y^{\prime}\right\}$,

2. $D^{\prime}=\varphi^{-1}(D)$, and

3. if $C_{y^{\prime}}$ denotes the residue curve of $Y^{\prime}$ and $\varphi_{y^{\prime}}: C_{y^{\prime}} \rightarrow C_{y}$ is the morphism induced by $\varphi$, then $\varphi_{y^{\prime}}$ is tamely ramified and is branched only over the points of $C_{y}$ corresponding to tangent directions at $y$ represented by edges in $\Sigma_{0}$. 
Remark 6.13. The morphism $\varphi$ is necessarily flat and its degree is equal to the degree of $\varphi_{y^{\prime}}$.

Example 6.14. Let $\varphi:\left(X^{\prime}, V^{\prime}, D^{\prime}\right) \rightarrow(X, V, D)$ be a tame covering of triangulated punctured curves. Suppose that $V$ and $V^{\prime}$ are strongly semistable vertex sets. Let $\Sigma=$ $\Sigma(X, V \cup D)$ and $\Sigma^{\prime}=\Sigma\left(X^{\prime}, V^{\prime} \cup D^{\prime}\right)=\varphi^{-1}(\Sigma)$. Let $x \in V$ be a finite vertex of $\Sigma$, let $e_{1}, \ldots, e_{r}$ be the finite edges of $\Sigma$ adjacent to $x$, let $\Sigma_{0}=\{x\} \cup \bigcup_{i=1}^{r} e_{i}^{\circ}$, let $Y=\tau^{-1}\left(\Sigma_{0}\right)$, and let $D_{0}=D \cap Y$. Then, $\left(Y, x, D_{0}\right)$ is a punctured star-shaped curve. Let $\Sigma_{0}^{\prime}$ be a connected component of $\varphi^{-1}\left(\Sigma_{0}\right)$, let $x^{\prime} \in \Sigma_{0}^{\prime}$ be the unique inverse image of $x$, let $Y^{\prime}=\tau^{-1}\left(\Sigma_{0}^{\prime}\right)$, and let $D_{0}^{\prime}=D^{\prime} \cap Y^{\prime}$. Then, $\left(Y^{\prime}, x^{\prime}, D_{0}^{\prime}\right)$ is also a punctured star-shaped curve, and $\varphi$ restricts to a finite morphism $\varphi: Y^{\prime} \rightarrow Y$. This is in fact a tame covering of punctured star-shaped curves by Remark 4.34 and Proposition 4.35 .

Proposition 6.15. Let $\varphi:\left(Y^{\prime}, y^{\prime}, D^{\prime}\right) \rightarrow(Y, y, D)$ be a degree- $\delta$ tame covering of punctured star-shaped curves. ${ }^{\mathrm{b}}$ Let $\Sigma_{0}=\Sigma(Y,\{y\} \cup D)$, let $\Sigma_{0}^{\prime}=\Sigma\left(Y^{\prime},\left\{y^{\prime}\right\} \cup D^{\prime}\right)$, let $C_{y}\left(\right.$ resp. $\left.C_{y^{\prime}}\right)$ be the residue curve of $Y\left(\right.$ resp. $\left.Y^{\prime}\right)$, and let $\varphi_{y^{\prime}}: C_{y^{\prime}} \rightarrow C_{y}$ be the induced morphism.

1. Let $B$ be a connected component of $Y \backslash\{y\}$ disjoint from $\Sigma_{0}$. Then, $\varphi^{-1}$ is a disjoint union of $\delta$ open balls mapping isomorphically onto $B$.

2. Let $B$ be a connected component of $Y \backslash\{y\}$ meeting $D$ and choose an isomorphism of $B$ with $\mathbf{B}(1)_{+}$which identifies the unique point of $B \cap D$ with 0 . Let $B^{\prime}$ be a connected component of $\varphi^{-1}(B)$. Then, $\left.\varphi\right|_{B^{\prime}}: B^{\prime} \rightarrow B$ is a finite morphism, the degree of $\left.\varphi\right|_{B^{\prime}}$ is the ramification degree $\delta^{\prime}$ of $\varphi_{y^{\prime}}$ at $\operatorname{red}\left(B^{\prime}\right)$, and there is an isomorphism $B^{\prime} \cong \mathbf{B}(1)_{+}$sending the unique point of $B^{\prime} \cap D^{\prime}$ to 0 such that the composition

$$
\begin{aligned}
& \quad \mathbf{B}(1)_{+} \cong B^{\prime} \stackrel{\varphi}{\longrightarrow} B \cong \mathbf{B}(1)_{+} \\
& \text {is } t \mapsto t^{\delta^{\prime}} .
\end{aligned}
$$

3. Let $A$ be an open annulus connected component of $Y \backslash\{y\}$ and choose an isomorphism $A \cong \mathbf{S}(a)_{+}$. Let $A^{\prime}$ be a connected component of $\varphi^{-1}(A)$. Then, $\left.\varphi\right|_{A^{\prime}}: A^{\prime} \rightarrow A$ is a finite morphism, the degree of $\left.\varphi\right|_{A^{\prime}}$ is the ramification degree $\delta^{\prime}$ of $\varphi_{y^{\prime}}$ at red $\left(A^{\prime}\right)$, and there is an isomorphism of $A^{\prime}$ with an open annulus $\mathbf{S}\left(a^{\prime}\right)$ such that the composition

$$
\begin{aligned}
& \quad \mathbf{S}\left(a^{\prime}\right)_{+} \cong A^{\prime} \stackrel{\varphi}{\longrightarrow} A \cong \mathbf{S}(a)_{+} \\
& \text {is } t \mapsto t^{\delta^{\prime}} . \\
& \text { 4. } \varphi \text { is étale over } Y \backslash D . \\
& \text { 5. } \varphi \varphi^{-1}\left(\Sigma_{0}\right)=\Sigma_{0}^{\prime} .
\end{aligned}
$$

Proof. In the situation of (1), let $\bar{x}=\operatorname{red}(B)$. Since $\varphi_{y^{\prime}}$ is not branched over $\bar{x}$, there are $\delta$ distinct points of $C_{y^{\prime}}$ mapping to $\bar{x}$; hence, there are $\delta$ connected components $B_{1}^{\prime}, \ldots, B_{\delta}^{\prime}$ of $Y^{\prime} \backslash\left\{y^{\prime}\right\}$ mapping onto $B$. The restriction of $\varphi$ to each $B_{i}^{\prime}$ is finite of degree 1 .

Next, we prove (2). It is clear that $\left.\varphi\right|_{B^{\prime}}: B^{\prime} \rightarrow B$ is finite, hence surjective; since $B^{\prime}$ is a connected component of $Y^{\prime} \backslash\left\{y^{\prime}\right\}$ containing a point of $D^{\prime}=\varphi^{-1}(D)$, it is an open ball. Let $x$ (resp. $x^{\prime}$ ) be the unique point of $D$ (resp. $\left.D^{\prime}\right)$ contained in $B$ (resp. $\left.B^{\prime}\right)$, and let $e \subset \Sigma_{0}$ (resp. $e^{\prime} \subset \Sigma_{0}^{\prime}$ ) be the edge adjacent to $x$ (resp. $x^{\prime}$ ). Then, $B^{\prime} \backslash\left\{x^{\prime}\right\} \rightarrow B \backslash$ $\{x\}$ is a finite morphism of punctured open balls, so by Lemma 3.3, Proposition 4.8, and 
Theorem 4.23(2), the restriction of $\varphi$ to $e^{\prime}$ is affine morphism $e^{\prime} \rightarrow e$ of degree $\delta^{\prime}$, and $\delta^{\prime}$ is the degree of $B^{\prime} \rightarrow B$. So by Lemma 6.10 , there is an isomorphism $B^{\prime} \cong \mathbf{B}(1)_{+}$as described in the statement of the Theorem.

In the situation of (3), we claim that $A^{\prime}$ is an open annulus. Clearly, $\left.\varphi\right|_{A^{\prime}}: A^{\prime} \rightarrow A$ is finite, hence surjective. If $A^{\prime}$ is not an open annulus, then $A^{\prime} \cong \mathbf{B}(1)_{+}$is an open ball. The morphism $\mathbf{B}(1)_{+} \cong A^{\prime} \rightarrow A \cong \mathbf{S}(a)_{+}$is given by a unit on $\mathbf{B}(1)_{+}$, which has constant absolute value; this contradicts surjectivity, so $A^{\prime}$ is in fact an open annulus. The proof now proceeds exactly as above.

Parts (4) and (5) follow immediately from parts (1) to (3).

The following proposition is reminiscent of [42], Proposition 3.3.2; see [15], Theorem 6.3.9.

Corollary 6.16. Let $\varphi:\left(Y^{\prime}, y^{\prime}, D_{0}^{\prime}\right) \rightarrow\left(Y, y, D_{0}\right)$ be a tame covering of punctured starshaped curves, and let $i:(Y, y) \hookrightarrow(X, x)$ be a compactification of $Y$. Let $D_{1}$ be the union of $i\left(D_{0}\right)$ with a choice of $K$-point from every connected component of $X \backslash i(Y)$. Then, there exists a compactification $i^{\prime}:\left(Y^{\prime}, y^{\prime}\right) \hookrightarrow\left(X^{\prime}, x^{\prime}\right)$ and a tame covering $\psi:\left(X^{\prime}, x^{\prime}, D_{1}^{\prime}\right) \rightarrow$ $\left(X, x, D_{1}\right)$ such that $\psi \circ i^{\prime}=i \circ \varphi$.

Proof. We compactify $\left(Y^{\prime}, y^{\prime}\right)$ as in the proof of Proposition 6.5, gluing balls onto the annulus connected components of $Y^{\prime} \backslash\left\{y^{\prime}\right\}$. By Proposition 6.15(3), if $A \cong \mathbf{S}(a)_{+}$is an open annulus connected component of $Y \backslash\{y\}$ and $A^{\prime} \subset Y^{\prime} \backslash\left\{y^{\prime}\right\}$ is a connected component mapping to $A$, then we can choose an isomorphism $A^{\prime} \cong \mathbf{S}\left(a^{\prime}\right)_{+}$such that $\mathbf{S}\left(a^{\prime}\right)_{+} \cong A^{\prime} \rightarrow$ $A \cong \mathbf{S}(a)_{+}$is of the form $t \mapsto t^{\delta}$; this map extends to a morphism $\mathbf{B}(1)_{+} \rightarrow \mathbf{B}(1)_{+}$, and these maps glue to give a tame covering $X^{\prime} \rightarrow X$.

\subsection{A local lifting theorem}

Let $(Y, y, D)$ be a punctured star-shaped curve with skeleton $\Sigma_{0}=\Sigma(Y,\{y\} \cup D)$ and residue curve $C_{y}$. Let $C^{\prime}$ be a smooth, proper, connected $k$-curve, and let $\bar{\varphi}: C^{\prime} \rightarrow C_{y}$ be a finite, tamely ramified morphism branched only over the points of $C_{y}$ corresponding to tangent directions at $y$ represented by edges in $\Sigma_{0}$. A lifting of $C^{\prime}$ to a punctured starshaped curve over $(Y, y, D)$ is the data of a punctured star-shaped curve $\left(Y^{\prime}, y^{\prime}, D^{\prime}\right)$, a tame covering $\varphi:\left(Y^{\prime}, y^{\prime}, D^{\prime}\right) \rightarrow(Y, y, D)$, and an isomorphism of the residue curve $C_{y^{\prime}}$ with $C^{\prime}$ which identifies $\bar{\varphi}$ with the morphism $\varphi_{y^{\prime}}: C_{y^{\prime}} \rightarrow C_{y}$ induced by $\varphi$. An isomorphism between two liftings $\left(Y^{\prime}, y^{\prime}\right)$ and $\left(Y^{\prime \prime}, y^{\prime \prime}\right)$ is a $Y$-isomorphism $Y^{\prime} \rightarrow Y^{\prime \prime}$ such that the induced morphism $C_{y^{\prime}} \stackrel{\sim}{\longrightarrow} C_{y^{\prime \prime}}$ respects the identifications $C_{y^{\prime}} \cong C^{\prime}$ and $C_{y^{\prime \prime}} \cong C^{\prime}$.

Theorem 6.18. Let $(Y, y, D)$ be a punctured star-shaped curve with skeleton $\Sigma_{0}=$ $\Sigma(Y,\{y\} \cup D)$ and residue curve $C_{y}$, let $C^{\prime}$ be a smooth, proper, connected $k$-curve, and let $\bar{\varphi}: C^{\prime} \rightarrow C_{y}$ be a finite, tamely ramified morphism branched only over the points of $C_{y}$ corresponding to tangent directions at y represented by edges in $\Sigma_{0}$. Then, there exists a lifting of $C^{\prime}$ to a punctured star-shaped curve over $(Y, y, D)$; and this lifting is unique up to unique isomorphism.

Before giving the proof of this theorem, we need a technical lemma. Let $\mathcal{X}$ be a finitely presented, flat, separated $R$ scheme, and let $\mathfrak{X}$ be its $\varpi$-adic completion; this is 
an admissible formal $R$ scheme by [11], Proposition 3.12. There is a canonical morphism $i_{\mathcal{X}}: \mathfrak{X}_{K} \rightarrow \mathcal{X}_{K}^{\text {an }}$ defined in [22], §A.3, which identifies $\mathfrak{X}_{K}$ with a compact analytic domain in $\mathcal{X}_{K}^{\text {an }}$, which is functorial in $\mathcal{X}$ and respects the formation of fiber products, and which is an isomorphism when $\mathcal{X}$ is proper over $R$. (This fact is implicitly contained in the statement of Lemma 5.1.)

Lemma 6.19. Let $\mathcal{X}, \mathcal{X}^{\prime}$ be finitely presented, flat, separated $R$ schemes and let $\mathfrak{X}, \mathfrak{X}^{\prime}$ denote their $\varpi$-adic completions. Let $\mathcal{X}^{\prime} \rightarrow \mathcal{X}$ be a finite and flat morphism. Then the square

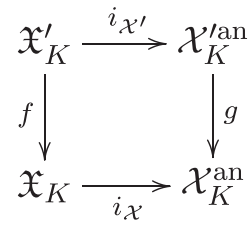

is Cartesian.

Proof. ' The vertical arrows $f, g$ of Equation 6.19 .1 are finite, and the horizontal arrows $i_{\mathcal{X}^{\prime}}, i_{\mathcal{X}}$ are immersions of analytic domains. Identifying $\mathfrak{X}_{K}$ and $\mathfrak{X}_{K}^{\prime}$ with their images in $\mathcal{X}_{K}^{\text {an }}$ and $\mathcal{X}_{K}^{\prime a n}$, then it suffices to show that $g^{-1}\left(\mathfrak{X}_{K}\right) \subset \mathfrak{X}_{K}^{\prime}$. Let $x^{\prime} \in g^{-1}\left(\mathfrak{X}_{K}\right)$, and let $x=$ $g\left(x^{\prime}\right) \in \mathfrak{X}_{K}$. Then, $x$ has a reduction in $\mathfrak{X}_{k}=\mathcal{X}_{k}$, so the morphism $x: \operatorname{Spec}(\mathscr{H}(x)) \rightarrow \mathcal{X}_{K}$ extends to $\operatorname{Spec}(\mathscr{H}(x)) \rightarrow \mathcal{X}$. By the valuative criterion of properness, as applied to the composition $\operatorname{Spec}\left(\stackrel{\mathscr{H}}{\mathscr{H}}\left(x^{\prime}\right)\right) \rightarrow \operatorname{Spec}(\stackrel{\mathscr{H}}{\mathscr{H}}(x)) \rightarrow \mathcal{X}$, we see that $x^{\prime}: \operatorname{Spec}\left(\mathscr{H}\left(x^{\prime}\right)\right) \rightarrow \mathcal{X}_{K}^{\prime}$ extends to $\operatorname{Spec}\left(\stackrel{\mathscr{H}}{\mathscr{C}}\left(x^{\prime}\right)\right)$. Therefore, $x^{\prime}$ has a reduction in $\mathfrak{X}_{k}^{\prime}$, so $x^{\prime} \in \mathfrak{X}_{K}^{\prime}$.

\subsection{Proof of Theorem 6.18}

We first prove the theorem when $Y$ is proper. In this case, we may and do assume that $Y$ is the analytic generic fiber of a smooth, proper, connected formal curve $\mathfrak{X}$ over $\operatorname{Spf}(R)$. Let $\mathcal{X} \rightarrow \operatorname{Spec}(R)$ be the algebraization of $\mathfrak{X}$ (Lemma 5.1); this is a smooth, proper relative curve of finite presentation and with connected fibers whose $\varpi$-adic completion is isomorphic to $\mathfrak{X}$. Note that $\mathcal{X}_{k}=\mathfrak{X}_{k}=C_{y}$. Let $X=\mathcal{X}_{K}$, so $X^{\text {an }}=\mathfrak{X}_{K}=Y$. By the valuative criterion of properness, every point $x$ of $D$ extends uniquely to a $\operatorname{section} \operatorname{Spec}(R) \rightarrow \mathcal{X}$ which sends the closed point to the reduction of $x$; hence, the closure $\mathcal{D}$ of $D$ in $\mathcal{X}$ is a disjoint union of sections. Let $\mathcal{U}=\mathcal{X} \backslash \mathcal{D}$.

The theory of the tamely ramified étale fundamental group $\pi_{1}^{t}$ of a morphism of schemes with a relative normal crossing divisor is developed in [40], Exposé XIII. The finite-index subgroups of $\pi_{1}^{t}$ classify tamely ramified étale covers. The subscheme $\mathcal{D} \subset \mathcal{X}$ is a relative normal crossing divisor relative to $\operatorname{Spec}(R)$, so the proof of Corollaire 2.12 of loc. cit. shows that the specialization homomorphism $\pi_{1}^{t}\left(\mathcal{U}_{K}\right) \rightarrow \pi_{1}^{t}\left(\mathcal{U}_{k}\right)$ is surjective (we suppress the base points). Moreover, by [40], Lemme XIII.2.11, a generator $\sigma_{x}$ for the inertia group at a point $x \in D$ specializes under $\pi_{1}^{t}\left(\mathcal{U}_{K}\right) \rightarrow \pi_{1}^{t}\left(\mathcal{U}_{k}\right)$ to a generator $\sigma_{\text {red }(x)}$ for the inertia group at its reduction.

Let $\bar{D}^{\prime}=\bar{\varphi}^{-1}\left(\mathcal{D}_{k}\right)$, and let $\bar{U}^{\prime}=C^{\prime} \backslash \bar{D}^{\prime}$; so $\bar{\varphi}: \bar{U}^{\prime} \rightarrow \mathcal{U}_{k}$ is a tamely ramified étale cover of $\mathcal{U}_{k}$ over $\mathcal{X}_{k}$ relative to $\mathcal{D}_{k}$. Let $\varphi: \mathcal{U}^{\prime} \rightarrow \mathcal{U}$ be the unique lift to an étale cover of $\mathcal{U}$, and let $U^{\prime}=\mathcal{U}_{K}^{\prime}$ and $U=\mathcal{U}_{K}=X \backslash D$. Then, $\bar{U}^{\prime} \rightarrow \mathcal{U}_{k}$ corresponds to a finite-index subgroup $\bar{H} \leq \pi_{1}^{t}\left(\mathcal{U}_{k}\right)$; and due to the surjectivity of the specialization 
homomorphism $\pi_{1}^{t}(U) \rightarrow \pi_{1}^{t}\left(\mathcal{U}_{k}\right)$, the generic fiber $U^{\prime}$ is connected and $\varphi_{K}: U^{\prime} \rightarrow U$ corresponds to the inverse image $H \leq \pi_{1}^{t}(U)$ of $\bar{H}$. Let $X^{\prime}$ be the smooth compactification of $U^{\prime}$; by abuse of notation, we also let $\varphi_{K}: X^{\prime} \rightarrow X$ denote the morphism extending $\varphi_{K}: U^{\prime} \rightarrow U$. The ramification profile of $\varphi_{K}$ over a point $x \in D$ is determined by the cycle decomposition of the action of the inertia element $\sigma_{x}$ on the coset space $\pi_{1}^{t}(U) / H$. Since $\pi_{1}^{t}(U) / H \stackrel{\sim}{\longrightarrow} \pi_{1}^{t}\left(\mathcal{U}_{k}\right) / \bar{H}$ and $\sigma_{x}$ specializes to $\sigma_{\text {red }(x)}$, it follows that the ramification profiles of $\varphi_{K}: X^{\prime} \rightarrow X$ above $x$ and of $\bar{\varphi}: C^{\prime} \rightarrow \mathcal{X}_{k}=C_{y}$ above red $(x)$ are identical. Applying the Riemann-Hurwitz formula to $\varphi_{K}$ and to $\bar{\varphi}$ shows that the genus of $X^{\prime}$ equals the genus of $C^{\prime}$.

Letting $\mathfrak{U}$ and $\mathfrak{U}^{\prime}$ denote the $\varpi$-adic completions of $\mathcal{U}$ and $\mathcal{U}^{\prime}$, respectively, we have a Cartesian square

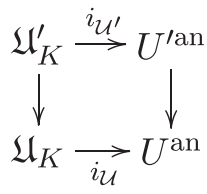

by Lemma 6.19. In other words, $\mathfrak{U}_{K}^{\prime}$ is the inverse image of $\mathfrak{U}_{K}$ under $\varphi_{K}: U^{\prime \text { an }} \rightarrow U^{\text {an }}$. Let $y^{\prime} \in \mathfrak{U}_{K}^{\prime}$ be the unique point reducing to the generic point of $\mathfrak{U}_{k}^{\prime}=\bar{U}^{\prime}$. By functoriality of the reduction map, this is the unique point of $\mathfrak{U}_{K}^{\prime}$ mapping to $y$ since the map $\mathfrak{U}_{k}^{\prime} \rightarrow \mathfrak{U}_{k}$ is dominant. Therefore, $\varphi_{K}^{-1}(y)=\left\{y^{\prime}\right\}$. Moreover, since the genus of the point $y^{\prime}$ is equal to the genus of $C^{\prime}$ (the smooth compactification of $\mathfrak{U}_{k}^{\prime}$ ), which coincides with the genus of $X^{\prime}$ by the genus formula ([12], (4.1)), we see that $\left\{y^{\prime}\right\}$ is the minimal skeleton of $X^{\prime}$. In particular, $X^{\prime}$ has a smooth formal model $\mathfrak{X}^{\prime}$ with special fiber $C^{\prime}=C_{y^{\prime}}$, and $X^{\prime} \rightarrow X$ extends to a finite morphism $\mathfrak{X}^{\prime} \rightarrow \mathfrak{X}$ lifting $\bar{\varphi}: C^{\prime} \rightarrow C_{y}$. Let $\varphi: \mathcal{X}^{\prime} \rightarrow \mathcal{X}$ be the algebraization of this morphism. The restriction of $\varphi$ to the inverse image of $\mathcal{U}$ is the unique étale morphism extending $\bar{U}^{\prime} \rightarrow \mathfrak{U}_{k}$, so $\mathcal{X}^{\prime} \rightarrow \mathcal{X}$ is in fact a compactification of the morphism $\varphi: \mathcal{U}^{\prime} \rightarrow \mathcal{U}$ above.

Let $D^{\prime}=\varphi_{K}^{-1}(D) \subset X^{\prime}$, and let $Y^{\prime}=X^{\prime a n}$. In order to show that $\left(Y^{\prime}, y^{\prime}, D^{\prime}\right)$ is a punctured star-shaped curve, we must argue that $D^{\prime}$ is a compatible divisor in $Y^{\prime}$, i.e. that distinct points of $D^{\prime}$ reduce to distinct points of $C_{y^{\prime}}=C^{\prime}$. Suppose not, so there are two points $x_{1}^{\prime}, x_{2}^{\prime} \in D^{\prime}$ with the same reduction $\bar{x} \in \bar{D}^{\prime}$. Let $\bar{x}=\operatorname{red}\left(x^{\prime}\right) \in \mathcal{D}_{k}$. Since distinct points of $D$ have distinct reductions in $\mathcal{D}_{k}$, by functoriality of the reduction map, we have $\varphi_{K}\left(x_{1}^{\prime}\right)=$ $\varphi_{K}\left(x_{2}^{\prime}\right)=x \in D$, the unique point reducing to $\bar{x}$. Since the ramification profiles of $\bar{\varphi}$ over $\bar{x}$ and of $\varphi_{K}$ over $x$ are the same, the finite sets $\bar{\varphi}^{-1}(x) \subset \bar{D}^{\prime}$ and $\varphi_{K}^{-1}(x) \subset D^{\prime}$ have the same cardinality; so by the pigeonhole principle, there exists some $\bar{x}^{\prime \prime} \in \bar{\varphi}^{-1}(\bar{x})$ which is not the reduction of any point of $D^{\prime}$. Then, the formal fiber $\operatorname{red}^{-1}\left(\vec{x}^{\prime \prime}\right) \subset Y^{\prime} \backslash D^{\prime}$ is an open ball connected component of $Y^{\prime} \backslash\left\{y^{\prime}\right\}$, and $\operatorname{red}^{-1}\left(x^{\prime \prime}\right) \rightarrow \operatorname{red}^{-1}(x) \backslash\{x\}$ is a finite (surjective) morphism to a punctured open ball, which contradicts Lemma 4.3. Therefore, $\left(Y^{\prime}, y^{\prime}, D^{\prime}\right)$ is a punctured star-shaped curve and $\varphi:\left(Y^{\prime}, y^{\prime}, D^{\prime}\right) \rightarrow(Y, y, D)$ is a tame covering.

Now, we briefly address uniqueness of the lift $\varphi:\left(Y^{\prime}, y^{\prime}, D^{\prime}\right) \rightarrow(Y, y, D)$, still in the case when $Y$ is proper. Given such a lift, running parts of the above argument backward, one sees that $Y^{\prime} \cong X^{\prime a n}$ where $X^{\prime}$ is the smooth compactification of the generic fiber of the unique étale extension $\mathcal{U}^{\prime} \rightarrow \mathcal{U}$ of $\bar{U}^{\prime} \rightarrow \mathfrak{U}_{k}$. As all of these constructions are unique up to unique isomorphism, the same is true of $Y^{\prime}$. 
Now, suppose that $(Y, y)$ is not proper. Let $Y \hookrightarrow X$ be a compactification (6) of $Y$; we will identify $Y$ with its image in $X$. Let $D_{1}$ be the union of $D$ with a choice of one $K$-point from every connected component of $X \backslash Y$. Let $\varphi:\left(X^{\prime}, y^{\prime}, D_{1}^{\prime}\right) \rightarrow$ $\left(X, y, D_{1}\right)$ be a lifting of $C^{\prime}$ to a punctured star-shaped curve over $\left(X, y, D_{1}\right)$, and let $Y^{\prime}=\varphi^{-1}(Y)$. Then, $\left(Y^{\prime}, y^{\prime}, \varphi^{-1}(D)\right)$ is a lifting of $C^{\prime}$ to a punctured star-shaped curve over $(Y, y, D)$.

Let $\psi: Y^{\prime} \rightarrow Y^{\prime}$ be an automorphism of $Y^{\prime}$ as a lifting of $C^{\prime}$. Since $\psi$ induces the identity map $C_{y^{\prime}} \rightarrow C_{y^{\prime}}$ on residue curves, $\psi$ takes each connected component of $Y^{\prime} \backslash\left\{y^{\prime}\right\}$ to itself. Recall that $X^{\prime} \backslash Y^{\prime}$ consists of a disjoint union of closed balls around the points of $D_{1}^{\prime} \backslash D^{\prime}$, where $D_{1}^{\prime}=\varphi^{-1}\left(D_{1}\right)$. Let $x^{\prime} \in D_{1}^{\prime} \backslash D^{\prime}$, let $B^{\prime}$ be the connected component of $X^{\prime} \backslash\left\{y^{\prime}\right\}$ containing $x^{\prime}$, and let $B=\varphi\left(B^{\prime}\right) \subset X$. By Proposition 6.15(2), we can choose isomorphisms $B \cong \mathbf{B}(1)_{+}$and $B^{\prime} \cong \mathbf{B}(1)_{+}$sending $x^{\prime}$ and $\varphi\left(x^{\prime}\right)$ to 0 , such that the composition

$$
\mathbf{B}(1)_{+} \cong B^{\prime} \stackrel{\varphi}{\longrightarrow} B \cong \mathbf{B}(1)_{+}
$$

is of the form $t \mapsto t^{\delta}$. Let $A^{\prime}=Y^{\prime} \cap B^{\prime}$, and let $A=Y \cap B=\varphi\left(A^{\prime}\right)$. The isomorphism $B \cong \mathbf{B}(1)_{+}$(resp. $\left.B^{\prime} \cong \mathbf{B}(1)_{+}\right)$identifies $A$ (resp. $A^{\prime}$ ) with an open annulus $\mathbf{S}(a)_{+}$(resp. $\left.\mathbf{S}\left(a^{\prime}\right)_{+}\right)$in $\mathbf{B}(1)_{+}$. Since $\left.\psi\right|_{A^{\prime}}: A^{\prime} \rightarrow A^{\prime}$ is an $A$-morphism, the composition $\mathbf{S}\left(a^{\prime}\right)_{+} \cong$ $A^{\prime} \rightarrow A^{\prime} \cong \mathbf{S}\left(a^{\prime}\right)_{+}$is of the form $t \mapsto \zeta t$, where $\zeta \in R^{\times}$is a $\delta$ th root of unity. Therefore, $\left.\psi\right|_{A^{\prime}}$ extends uniquely to a $B$-morphism $\left.\psi\right|_{B^{\prime}}: B^{\prime} \rightarrow B^{\prime}$ fixing 0 . Gluing these morphisms together, we obtain an $X$-morphism $\psi: X^{\prime} \rightarrow X^{\prime}$ extending $\psi: Y^{\prime} \rightarrow Y^{\prime}$. By construction, this is an automorphism of $X^{\prime}$ as a lifting of $C^{\prime}$ to $X$, which is thus the identity. Therefore, $\psi: Y^{\prime} \rightarrow Y^{\prime}$ is the identity.

Let $\varphi^{\prime}:\left(Y^{\prime \prime}, y^{\prime \prime}, D^{\prime \prime}\right) \rightarrow(Y, y, D)$ be another lifting of $C^{\prime}$ to a punctured star-shaped curve over $(Y, y, D)$. By Corollary 6.16, there exists a compactification $Y^{\prime \prime} \hookrightarrow X^{\prime \prime}$ of $Y^{\prime \prime}$ and an extension of $\varphi^{\prime}$ to a tame covering $\varphi^{\prime}:\left(X^{\prime \prime}, y^{\prime \prime}, \varphi^{\prime-1}\left(D_{1}\right)\right) \rightarrow\left(X, y, D_{1}\right)$. This is another lifting of $C^{\prime}$ to a punctured star-shaped curve over $\left(X, y, D_{1}\right)$, so there is an isomorphism $\psi:\left(X^{\prime}, y^{\prime}\right) \stackrel{\sim}{\longrightarrow}\left(X^{\prime \prime}, y^{\prime \prime}\right)$ of liftings. Since $Y^{\prime}=\varphi^{-1}(Y)$ and $Y^{\prime \prime}=\varphi^{\prime-1}(Y)$, the isomorphism $\psi$ restricts to an isomorphism $\left(Y^{\prime}, y^{\prime}\right) \stackrel{\sim}{\longrightarrow}\left(Y^{\prime \prime}, y^{\prime \prime}\right)$ of liftings.

Corollary 6.21. With the notation in Theorem 6.18, let $\left(Y^{\prime}, y^{\prime}, D^{\prime}\right) \rightarrow(Y, y, D)$ be a lifting of $C^{\prime}$ to a punctured star-shaped curve over $(Y, y, D)$. Then, the natural homomorphism

$$
\operatorname{Aut}_{Y}\left(Y^{\prime}\right) \longrightarrow \operatorname{Aut}_{C_{y}}\left(C^{\prime}\right)
$$

is bijective. If $\left(Y^{\prime \prime}, y^{\prime \prime}, D^{\prime \prime}\right) \rightarrow(Y, y, D)$ is a second lifting of $C^{\prime}$ to a punctured star-shaped curve over $(Y, y, D)$, then the natural map

$$
\operatorname{Isom}_{Y}\left(Y^{\prime \prime}, Y^{\prime}\right) \longrightarrow \operatorname{Isom}_{C_{y}}\left(C_{y^{\prime \prime}}, C_{y^{\prime}}\right)
$$

is bijective.

\section{Classification of liftings of harmonic morphisms of metrized complexes}

Fix a triangulated punctured curve $(X, V \cup D)$ with skeleton $\Sigma=\Sigma(X, V \cup D)$, and let $\tau: X^{\text {an }} \rightarrow \Sigma$ be the canonical retraction. Throughout this section, we assume that $\Sigma$ has no loop edges. Let $\varphi: \Sigma^{\prime} \rightarrow \Sigma$ be a tame covering of metrized complexes of curves. A lifting of $\Sigma^{\prime}$ to a tame covering of $(X, V \cup D)$ is a tame covering of triangulated punctured 
curves $\varphi:\left(X^{\prime}, V^{\prime} \cup D^{\prime}\right) \rightarrow(X, V \cup D)$ (see (4.30)) equipped with a $\Sigma$-isomorphism $\varphi^{-1}(\Sigma) \cong \Sigma^{\prime}$ of metrized complexes of curves. Since $V^{\prime}=\varphi^{-1}(V)$ and $D^{\prime}=\varphi^{-1}(D)$, we will often denote a lifting simply by $X^{\prime}$. Let $\varphi: X^{\prime} \rightarrow X$ and $\varphi^{\prime}: X^{\prime \prime} \rightarrow X$ be two liftings of $\Sigma^{\prime}$, and let $\psi: X^{\prime} \stackrel{\sim}{\longrightarrow} X^{\prime \prime}$ be an $X$-isomorphism of curves. Then, $\psi$ restricts to a $\Sigma$-automorphism

$$
\left.\psi\right|_{\Sigma^{\prime}}: \Sigma^{\prime} \cong \varphi^{-1}(\Sigma) \stackrel{\sim}{\longrightarrow} \varphi^{\prime-1}(\Sigma) \cong \Sigma^{\prime} .
$$

We will consider liftings up to $X$-isomorphism preserving $\Sigma^{\prime}$, i.e. such that $\psi_{\Sigma^{\prime}}$ is the identity, and we will also consider liftings up to isomorphism as curves over $X$.

For every finite vertex $x \in V$, let $\Sigma(x)$ be the connected component of $x$ in $\{x\} \cup(\Sigma \backslash V)$, let $Y(x)=\tau^{-1}(\Sigma(x))$, and let $D(x)=D \cap Y(x)$, as in Example 6.14. Then, $(Y(x), x, D(x))$ is a punctured star-shaped curve. By Proposition 2.22, for every finite vertex $x^{\prime} \in V\left(\Sigma^{\prime}\right)$ lying above $x$, the morphism $\varphi_{x^{\prime}}: C_{x^{\prime}} \rightarrow C_{x}$ is finite, tamely ramified, and branched only over the points of $C_{x}$ corresponding to tangent directions at $x$ represented by edges of $\Sigma(x)$. Let $\psi\left(x^{\prime}\right):\left(Y^{\prime}\left(x^{\prime}\right), x^{\prime}, D\left(x^{\prime}\right)\right) \rightarrow(Y(x), x, D(x))$ be the unique lifting of $C_{x^{\prime}}$ to a punctured star-shaped curve over $(Y(x), x, D(x))$ provided by Theorem 6.18 , and let $\Sigma^{\prime}\left(x^{\prime}\right)=\psi\left(x^{\prime}\right)^{-1}(\Sigma(x))=\Sigma\left(Y^{\prime},\left\{x^{\prime}\right\} \cup D^{\prime}\left(x^{\prime}\right)\right)$. Then, $\Sigma^{\prime}\left(x^{\prime}\right)$ is canonically identified with the connected component of $x^{\prime}$ in $\left\{x^{\prime}\right\} \cup\left(\Sigma^{\prime} \backslash V_{f}\left(\Sigma^{\prime}\right)\right)$ in such a way that for every edge $e^{\prime}$ of $\Sigma^{\prime}\left(x^{\prime}\right)$, the point $\operatorname{red}_{x^{\prime}}\left(e^{\prime}\right) \in C_{x^{\prime}}(k)$ is identified with the pointred $\left(e^{\prime}\right)$ defined in (6). This induces an identification of $D^{\prime}\left(x^{\prime}\right)$ with $\varphi^{-1}(D) \cap \Sigma^{\prime}\left(x^{\prime}\right)$. Let $\tau_{x^{\prime}}$ be the canonical retraction $Y^{\prime}\left(x^{\prime}\right) \rightarrow \Sigma^{\prime}\left(x^{\prime}\right)$ defined in (6).

Let $e^{\prime} \in E_{f}\left(\Sigma^{\prime}\right)$ and $e=\varphi\left(e^{\prime}\right)$. Choose $a, a^{\prime} \in K^{\times}$with $\operatorname{val}(a)=\ell(e)$ and $\operatorname{val}\left(a^{\prime}\right)=$ $\ell\left(e^{\prime}\right)$. Let $x^{\prime}, y^{\prime}$ be the endpoints of $e^{\prime}$. By Proposition 6.15(3), we can choose isomorphisms $\tau^{-1}\left(e^{\circ}\right) \cong \mathbf{S}(a)_{+}$and $\tau_{x^{\prime}}^{-1}\left(e^{\prime \circ}\right) \cong \mathbf{S}\left(a^{\prime}\right)_{+}, \tau_{y^{\prime}}^{-1}\left(e^{\prime \circ}\right) \cong \mathbf{S}\left(a^{\prime}\right)_{+}$in such a way that the finite morphisms $\tau_{x^{\prime}}^{-1}\left(e^{\prime \circ}\right) \rightarrow \tau^{-1}\left(e^{\circ}\right)$ and $\tau_{y^{\prime}}^{-1}\left(e^{\circ}\right) \rightarrow \tau^{-1}\left(e^{\circ}\right)$ are given by $t \mapsto t^{d_{e^{\prime}}(\varphi)}$, where $d_{e^{\prime}}(\varphi)$ is the degree of the edge map $e^{\prime} \rightarrow e$. In particular, there exists a $\tau^{-1}\left(e^{\circ}\right)$-isomorphism $\tau_{x^{\prime}}^{-1}\left(e^{\prime \circ}\right) \stackrel{\sim}{\longrightarrow} \tau_{y^{\prime}}^{-1}\left(e^{\circ}\right)$. As explained in (6.9), there are canonical identifications

$$
\operatorname{Aut}_{\tau^{-1}\left(e^{\circ}\right)}\left(d_{e^{\prime}}(\varphi)\right):=\operatorname{Aut}_{\tau^{-1}\left(e^{\circ}\right)}\left(\tau_{x^{\prime}}^{-1}\left(e^{\prime \circ}\right)\right)=\operatorname{Aut}_{\tau^{-1}\left(e^{\circ}\right)}\left(\tau_{y^{\prime}}^{-1}\left(e^{\prime \circ}\right)\right) \cong \mathbf{Z} / d_{e^{\prime}}(\varphi) \mathbf{Z}
$$

hence the set of isomorphisms $\tau_{x^{\prime}}^{-1}\left(e^{\prime \circ}\right) \stackrel{\sim}{\longrightarrow} \tau_{y^{\prime}}^{-1}\left(e^{\prime \circ}\right)$ is a principal homogeneous space under the pre- or post-composition action of $\operatorname{Aut}_{\tau^{-1}\left(e^{\circ}\right)}\left(d_{e^{\prime}}(\varphi)\right)$.

Let $E_{f}^{ \pm}\left(\Sigma^{\prime}\right)$ denote the set of oriented finite edges of $\Sigma^{\prime}$, and for $e^{\prime} \in E_{f}^{ \pm}\left(\Sigma^{\prime}\right)$ let $\vec{e}$ denote the same edge with the opposite orientation. Let $\mathcal{G}\left(\Sigma^{\prime}, X\right)$ denote the set of tuples $\left(\Theta_{e^{\prime}}\right)_{e^{\prime} \in E_{f}^{ \pm}\left(\Sigma^{\prime}\right)}$ of isomorphisms

$$
\Theta_{e^{\prime}}: \tau_{x^{\prime}}^{-1}\left(e^{\prime \circ}\right) \stackrel{\sim}{\longrightarrow} \tau_{y^{\prime}}^{-1}\left(e^{\prime \circ}\right)
$$

such that $\Theta_{\vec{e}}=\Theta_{e^{\prime}}^{-1}$, where $e^{\prime}=\overrightarrow{x^{\prime} y^{\prime}}$. We call $\mathcal{G}\left(\Sigma^{\prime}, X\right)$ the set of gluing data for a lifting of $\Sigma^{\prime}$ to a tame covering of $(X, V \cup D)$, and we emphasize that $\mathcal{G}\left(\Sigma^{\prime}, X\right)$ is nonempty.

Let $\alpha \in \operatorname{Aut}_{\Sigma}\left(\Sigma^{\prime}\right)$, so $\alpha$ is a degree-1 finite harmonic morphism $\Sigma^{\prime} \stackrel{\sim}{\longrightarrow} \Sigma^{\prime}$ preserving $\Sigma^{\prime} \rightarrow \Sigma$. Let $x^{\prime} \in V_{f}\left(\Sigma^{\prime}\right)$, and let $x^{\prime \prime}=\alpha\left(x^{\prime}\right)$ and $x=\varphi\left(x^{\prime}\right)=\varphi\left(x^{\prime \prime}\right)$. Part of the data of 
$\alpha$ is a $C_{X}$-isomorphism $\alpha_{x^{\prime}}: C_{X^{\prime}} \stackrel{\sim}{\longrightarrow} C_{x^{\prime \prime}}$. By Corollary 6.21, there is a unique lift of $\alpha_{x^{\prime}}$ to a $Y(x)$-isomorphism $Y^{\prime}\left(x^{\prime}\right) \stackrel{\sim}{\longrightarrow} Y^{\prime}\left(x^{\prime \prime}\right)$ of punctured star-shaped curves inducing the isomorphism $C_{x^{\prime}} \stackrel{\sim}{\longrightarrow} C_{x^{\prime \prime}}$ on residue curves. Let $e^{\prime} \in E_{f}\left(\Sigma^{\prime}\right)$ be an edge adjacent to $x^{\prime}$, let $e^{\prime \prime}=\alpha\left(e^{\prime}\right)$, and let $e=\varphi\left(e^{\prime}\right)=\varphi\left(e^{\prime \prime}\right)$. Then, $\alpha_{x^{\prime}}$ restricts to an isomorphism

$$
\alpha_{x^{\prime}}: \tau_{x^{\prime}}^{-1}\left(e^{\prime \circ}\right) \stackrel{\sim}{\longrightarrow} \tau_{x^{\prime \prime}}^{-1}\left(e^{\prime \prime \circ}\right) \text {. }
$$

Define the conjugation action of $\operatorname{Aut}_{\Sigma}\left(\Sigma^{\prime}\right)$ on $\mathcal{G}\left(\Sigma^{\prime}, X\right)$ by the rule

$$
\alpha \cdot\left(\Theta_{e^{\prime}}\right)_{e^{\prime} \in E_{f}^{ \pm}\left(\Sigma^{\prime}\right)}=\left(\alpha_{y^{\prime}}^{-1} \circ \Theta_{\alpha\left(e^{\prime}\right)} \circ \alpha_{x^{\prime}}\right)_{e^{\prime} \in E_{f}^{ \pm}\left(\Sigma^{\prime}\right)}
$$

where $e^{\prime}=\overrightarrow{x^{\prime} y^{\prime}}$.

Theorem 7.4 (Classification of lifts of harmonic morphisms). Let $(X, V \cup D)$ be a triangulated punctured curve with skeleton $\Sigma=\Sigma(X, V \cup D)$. Assume that $\Sigma$ has no loop edges. Let $\varphi: \Sigma^{\prime} \rightarrow \Sigma$ be a tame covering of metrized compexes of curves.

1. There is a canonical bijection between the set of gluing data $\mathcal{G}\left(\Sigma^{\prime}, X\right)$ and the set of liftings of $\Sigma^{\prime}$ to a tame covering of $(X, V \cup D)$, up to $X$-isomorphism preserving $\Sigma^{\prime}$. In particular, there exists a lifting of $\Sigma^{\prime}$. Any such lifting has no nontrivial automorphisms which preserve $\Sigma^{\prime}$.

2. Two tuples of gluing data determine $X$-isomorphic curves if and only if they are in the same orbit under the conjugation action Equation 7.3.1. The stabilizer in $\operatorname{Aut}_{\Sigma}\left(\Sigma^{\prime}\right)$ of an element of $\mathcal{G}\left(\Sigma^{\prime}, X\right)$ is canonically isomorphic to the $X$-automorphism group of the associated curve.

Proof. Given $\left(\Theta_{e^{\prime}}\right) \in \mathcal{G}\left(\Sigma^{\prime}, X\right)$, one can glue the local lifts $\left\{Y\left(x^{\prime}\right)\right\}_{x^{\prime} \in V_{f}\left(\Sigma^{\prime}\right)}$ via the isomorphisms Equation 7.2.1 to obtain an analytic space which one easily verifies is smooth and proper, hence arises as the analytification of an algebraic curve $X^{\prime}$. Moreover, the morphisms $Y^{\prime}\left(x^{\prime}\right) \rightarrow Y(x)$ glue to give a morphism $X^{\prime a n} \rightarrow X^{\text {an }}$, which is the analytification of a morphism $\varphi: X^{\prime} \rightarrow X$. By construction, if $V^{\prime}=\varphi^{-1}(V)$ and $D^{\prime}=\varphi^{-1}(D)$, then $\left(X^{\prime}, V^{\prime} \cup D^{\prime}\right) \rightarrow(X, V \cup D)$ is a lifting of $\Sigma^{\prime}$ to a tame covering of $(X, V \cup D)$.

Now, let $\varphi:\left(X^{\prime}, V^{\prime} \cup D^{\prime}\right) \rightarrow(X, V \cup D)$ be any lifting of $\Sigma^{\prime}$ to a tame covering of $(X, V \cup D)$. As explained in Example 6.14, for every $x \in V$, the inverse image $\varphi^{-1}(Y(x))$ is a disjoint union of tame covers of the punctured star-shaped curve $(Y(x), x, D(x))$, one for each $x^{\prime} \in \varphi^{-1}(x)$. By Theorem 6.18, we have canonical identification $\varphi^{-1}(Y(x))=$ $\coprod_{x^{\prime} \mapsto x} Y\left(x^{\prime}\right)$. For $x^{\prime}, y^{\prime} \in V^{\prime}$, we have $Y\left(x^{\prime}\right) \cap Y\left(y^{\prime}\right)=\bigsqcup_{e^{\prime} \ni x^{\prime}, y^{\prime}} \tau^{-1}\left(e^{\circ}\right)$, where $\tau: X^{\prime a n} \rightarrow$ $\Sigma^{\prime}$ is the retraction. Hence, $X^{\prime a n}$ is obtained by pasting the local liftings $Y\left(x^{\prime}\right)$ via a choice of isomorphisms $\left(\Theta_{e^{\prime}}\right) \in \mathcal{G}\left(\Sigma^{\prime}, X\right)$.

Let $\psi: X^{\prime} \stackrel{\sim}{\longrightarrow} X^{\prime}$ be an $X$-automorphism preserving $\Sigma^{\prime}$. Since $\psi$ is the identity on the set $\Sigma^{\prime} \subset X^{\prime a n}$, for $x^{\prime} \in V^{\prime}$, we have $\psi\left(Y\left(x^{\prime}\right)\right)=Y\left(x^{\prime}\right)$. We also have that for $x^{\prime} \in V^{\prime}$ the map of residue curves $\psi_{x^{\prime}}: C_{x^{\prime}} \rightarrow C_{x^{\prime}}$ is the identity, so by Corollary 6.21, $\psi$ restricts to the identity morphism $Y\left(x^{\prime}\right) \rightarrow Y\left(x^{\prime}\right)$. Since $X^{\prime a n}=\bigcup_{x^{\prime} \in V^{\prime}} Y\left(x^{\prime}\right)$, we have that $\psi$ is the identity. It follows from this that the tuple $\left(\Theta_{e^{\prime}}\right)$ can be recovered from the class of $X^{\prime}$ modulo $X$-isomorphisms preserving $\Sigma^{\prime}$, so different gluing data give rise to curves which are not equivalent under such isomorphisms. This proves (1).

Let $\varphi:\left(X^{\prime}, V^{\prime} \cup D^{\prime}\right) \rightarrow(X, V \cup D)$ and $\varphi^{\prime \prime}:\left(X^{\prime \prime}, V^{\prime \prime} \cup D^{\prime \prime}\right) \rightarrow(X, V \cup D)$ be the liftings of $\Sigma^{\prime}$ associated to the tuples of gluing data $\left(\Theta_{e^{\prime}}^{\prime}\right),\left(\Theta_{e^{\prime}}^{\prime \prime}\right) \in \mathcal{G}\left(\Sigma^{\prime}, X\right)$, respectively. Suppose 
that there exists $\alpha \in \operatorname{Aut}_{\Sigma}\left(\Sigma^{\prime}\right)$ such that $\alpha \cdot\left(\Theta_{e^{\prime}}^{\prime}\right)=\left(\Theta_{e^{\prime}}^{\prime \prime}\right)$. Then, for all $e^{\prime}=\overrightarrow{x^{\prime} y^{\prime}} \in E_{f}^{ \pm}\left(\Sigma^{\prime}\right)$, we have

$$
\Theta_{\alpha\left(e^{\prime}\right)}^{\prime \prime} \circ \alpha_{x^{\prime}}=\alpha_{y^{\prime}} \circ \Theta_{e^{\prime}}^{\prime}
$$

so the $Y\left(\varphi\left(x^{\prime}\right)\right)$-isomorphisms $\alpha_{x^{\prime}}: Y\left(x^{\prime}\right) \stackrel{\sim}{\longrightarrow} Y\left(\alpha\left(x^{\prime}\right)\right)$ glue to give an $X$-isomorphism $\alpha: X^{\prime} \stackrel{\sim}{\longrightarrow} X^{\prime \prime}$. Conversely, the restriction of an $X$-isomorphism $\alpha: X^{\prime} \stackrel{\sim}{\longrightarrow} X^{\prime \prime}$ to $\Sigma^{\prime}$ is a $\Sigma$-automorphism of $\Sigma^{\prime}$. It is easy to see that these are inverse constructions. Taking $X^{\prime}=X^{\prime \prime}$ we have an injective homomorphism $\operatorname{Aut}_{X}\left(X^{\prime}\right) \hookrightarrow \operatorname{Aut}_{\Sigma}\left(\Sigma^{\prime}\right)$; it follows formally from the above considerations that its image is the stabilizer of $\left(\Theta_{e^{\prime}}^{\prime}\right)$.

Remark 7.5. Let $X^{\prime}$ be a lifting of $\Sigma^{\prime}$ to a tame covering of $(X, V \cup D)$. It follows from Theorem 7.4(1) that the natural homomorphism

$$
\operatorname{Aut}_{X}\left(X^{\prime}\right) \longrightarrow \operatorname{Aut}_{\Sigma}\left(\Sigma^{\prime}\right)
$$

is injective. It is not in general surjective: see Example 7.8 (where all the stabilizer groups of elements of $\mathcal{G}\left(\Sigma^{\prime}, X\right)$ are proper subgroups of $\left.\operatorname{Aut}_{\Sigma}\left(\Sigma^{\prime}\right)\right)$.

Remark 7.6. It is worth mentioning that the question of lifting (and classification of all possible liftings) in the wildly ramified case is more subtle and cannot be guaranteed in general. Some lifting results in the wildly ramified case are known: e.g., Liu proves in [33], Proposition 5.4, that any finite surjective generically étale admissible cover of proper semistable curves over an algebraically closed field $k$ lifts to a finite morphism of smooth proper curves over $K$. However, the same statement for metrized complexes cannot be true in general: consider the metrized complex $\mathcal{C}$ consisting of a single finite vertex $v$ and an infinite vertex $u$ attached to $v$ by an infinite edge $e$ with $C_{v} \cong \mathbf{P}_{k}^{1}$ and $\operatorname{red}_{v}(e)=\infty$. Consider the degree $p$ generically étale morphism of metrized complexes $\mathcal{C} \rightarrow \mathcal{C}$ which restricts to a degree $p$ Artin-Schreier cover $\varphi: \mathbf{P}_{k}^{1} \rightarrow \mathbf{P}_{k}^{1}$, étale over $\mathbf{A}_{k}^{1} \rightarrow \mathbf{A}_{k}^{1}$ and ramified at $\infty$ (with ramification index $p$ ) and which is linear of slope $p$ on the infinite edge $e$. This map cannot be lifted to a degree $p$ cover $\mathbf{P}_{K}^{1} \rightarrow \mathbf{P}_{K}^{1}$ in characteristic zero; otherwise, we would obtain a (connected) étale degree $p$ cover of $\mathbf{A}_{K}^{1}$ which is impossible since $\mathbf{A}_{K}^{1}$ is simply connected.

We now state a variant of Theorem 7.4 in which we allow loop edges.

Theorem 7.7. Let $(X, V \cup D)$ be a triangulated punctured $K$-curve, let $\Sigma=\Sigma(X, V \cup D)$, and let $\bar{\varphi}: \Sigma^{\prime} \rightarrow \Sigma$ be a tame covering of $\Lambda$-metrized complexes of $k$-curves. Then, there exists a tame covering $\varphi:\left(X^{\prime}, V^{\prime} \cup D^{\prime}\right) \rightarrow(X, V \cup D)$ of triangulated punctured $K$-curves lifting $\varphi$.

Proof. The only issue is that in Theorem 7.7, we merely require that $V$ is a semistable vertex set whereas; whereas in Theorem 7.4, we require it to be strongly semistable. However, we can modify $\Sigma$ by inserting a (valence 2) vertex $v$ along each loop edge, with $C_{v} \cong \mathbf{P}_{k}^{1}$, and with the marked points on $C_{v}$ being 0 and $\infty$. Call the resulting metrized complex $\widetilde{\Sigma}$, and let $\bar{V}_{0}$ denote the set of vertices which have been added to the vertex set $\bar{V}$ for $\Sigma$. We construct a new metrized complex $\widetilde{\Sigma}^{\prime}$ from $\Sigma^{\prime}$ by adding $\bar{V}_{0}^{\prime}:=\bar{\varphi}^{-1}\left(\bar{V}_{0}\right)$ 
to the vertex set $\bar{V}^{\prime}$ for $\Sigma^{\prime}$ and letting $C_{v^{\prime}}^{\prime} \cong \mathbf{P}_{k}^{1}$ for $v^{\prime} \in \bar{V}_{0}^{\prime}$, with the marked points on $C_{\nu^{\prime}}^{\prime}$ being 0 and $\infty$. We can extend the tame covering $\bar{\varphi}: \Sigma^{\prime} \rightarrow \Sigma$ to a tame covering $\widetilde{\Sigma}^{\prime} \rightarrow \widetilde{\Sigma}$ by letting the map from $C_{v^{\prime}}^{\prime}$ to $C_{\bar{\varphi}\left(v^{\prime}\right)}$, for $v^{\prime} \in \bar{V}_{0}^{\prime}$, be $z \mapsto z^{d}$, where $d$ is the local degree of $\bar{\varphi}$ along the loop edge corresponding to $v^{\prime}$. By Theorem 7.4, there is a tame covering $\left(X^{\prime}, V^{\prime} \cup V_{0}^{\prime} \cup D^{\prime}\right) \rightarrow\left(X, V \cup V_{0} \cup D\right)$ lifting $\widetilde{\Sigma}^{\prime} \rightarrow \widetilde{\Sigma}$, where $V_{0}^{\prime}$ (resp. $V_{0}$ ) corresponds to $\bar{V}_{0}^{\prime}$ (resp. $\bar{V}_{0}$ ). Removing the vertices in $V_{0}^{\prime}$ and $V_{0}$ gives a tame covering $\left(X^{\prime}, V^{\prime} \cup D^{\prime}\right) \rightarrow(X, V \cup D)$ lifting $\bar{\varphi}$.

Example 7.8. In this example, we suppose that $\operatorname{char}(k) \neq 2$. Let $E$ be a Tate curve over $K$, let $\Sigma$ be its (set-theoretic) skeleton, and let $\tau: E^{\text {an }} \rightarrow \Sigma$ be the canonical retraction. Let $U: \mathbf{G}_{m}^{\text {an }} / q^{\mathbf{Z}} \stackrel{\sim}{\longrightarrow} E^{\text {an }}$ be the Tate uniformization of $E^{\text {an }}$. The two-torsion subgroup of $E$ is $U(\{ \pm 1, \pm \sqrt{q}\})$; choose a square root of $q$, let $y=\tau(U(1))$ and $z=\tau(U(\sqrt{q}))$, and let $V=\{y, z\} \subset \Sigma$. This is a semistable vertex set of $E$ and $\Sigma=\Sigma(E, V)$ is the circle with circumference $\operatorname{val}(q)$; the points $y$ and $z$ are antipodal on $\Sigma$. Let $e_{1}, e_{2}$ be the edges of $\Sigma$; orient $e_{1}$ so that $y$ is the source vertex and $e_{2}$ so that $z$ is the source vertex. The residue curves $C_{y}$ and $C_{z}$ are both isomorphic to $\mathbf{P}_{k}^{1}$; fix isomorphisms $C_{y} \cong \mathbf{P}_{k}^{1}$ and $C_{z} \cong \mathbf{P}_{k}^{1}$ such that the tangent direction at a vertex in the direction of the outgoing (resp. incoming) edge corresponds to $\infty$ (resp. 0 ).

Let $\Sigma^{\prime}$ be a circle of circumference $\frac{1}{2} \operatorname{val}(q)$, let $V^{\prime}=\left\{y^{\prime}, z^{\prime}\right\}$ be a pair of antipodal points on $\Sigma^{\prime}$, and let $e_{1}^{\prime}=\overrightarrow{y^{\prime} z^{\prime}}$ and $e_{2}^{\prime}=\overrightarrow{z^{\prime} y^{\prime}}$ be the two edges of $\Sigma^{\prime}$, with the indicated orientations. Enrich $\Sigma^{\prime}$ with the structure of a metrized complex of curves by setting $C_{y^{\prime}}=C_{z^{\prime}}=\mathbf{P}_{k}^{1}$ and letting the tangent direction at a vertex in the direction of the outgoing (resp. incoming) edge correspond to $\infty$ (resp. 0). Define a morphism $\varphi: \Sigma^{\prime} \rightarrow \Sigma$ of metric graphs by

$$
\varphi\left(y^{\prime}\right)=y, \quad \varphi\left(z^{\prime}\right)=z, \quad \varphi\left(e_{1}^{\prime}\right)=e_{1}, \quad \varphi\left(e_{2}^{\prime}\right)=e_{2},
$$

with both degrees equal to 2 . See Figure 3 .

Topologically, $\Sigma^{\prime} \rightarrow \Sigma$ is a homeomorphism. Let $\varphi_{y^{\prime}}: C_{y^{\prime}} \rightarrow C_{y}$ and $\varphi_{z^{\prime}}: C_{z^{\prime}} \rightarrow C_{z}$ both be the morphism $t \mapsto t^{2}: \mathbf{P}_{k}^{1} \rightarrow \mathbf{P}_{k}^{1}$. This makes $\varphi$ into a degree- 2 tame covering of metrized complexes of curves.

For $x \in V$, the analytic space $Y(x)$ is an open annulus of logarithmic modulus $\operatorname{val}(q)$; fix an isomorphism $Y(x) \cong \mathbf{S}(q)_{+}$for each $x$. For $x^{\prime} \in V^{\prime}$, the analytic space $Y^{\prime}\left(x^{\prime}\right)$ is an open annulus of logarithmic modulus $\frac{1}{2} \operatorname{val}(q)$; fix isomorphisms $Y^{\prime}\left(x^{\prime}\right) \cong \mathbf{S}(\sqrt{q})_{+}$such that the morphisms $Y^{\prime}\left(x^{\prime}\right) \rightarrow Y\left(\varphi\left(x^{\prime}\right)\right)$ are given by $t \mapsto t^{2}: \mathbf{S}(\sqrt{q})_{+} \rightarrow \mathbf{S}(q)_{+}$. For $e^{\prime} \in E\left(\Sigma^{\prime}\right)$, we have $d_{\varphi}\left(e^{\prime}\right)=2$ by definition. Hence, $\mathcal{G}\left(\Sigma^{\prime}, E\right)$ has four elements, which

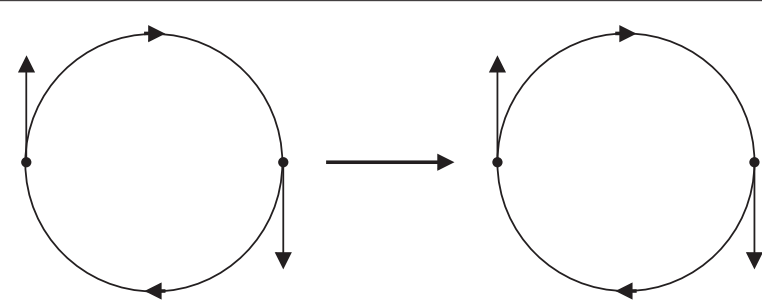

Figure 3 This figure illustrates Example 7.8. The morphism $\varphi$ is a homeomorphism of underlying sets but has a degree of 2 on $e_{1}^{\prime}$ and $e_{2}^{\prime}$. The arrows on $e_{1}, e_{2}, e_{1}^{\prime}, e_{2}^{\prime}$ represent the chosen orientations and the indicated tangent vectors at $y, z, y^{\prime}, z^{\prime}$ represent the tangent direction corresponding to $\infty$ in the corresponding $k$-curve. 
we label $( \pm 1, \pm 1)$. By Theorem 7.4(1), there are four corresponding classes of liftings of $\Sigma^{\prime}$ to a tame covering of $(E, V)$ up to isomorphism preserving $\Sigma^{\prime}$.

Any $\Sigma$-automorphism of $\Sigma^{\prime}$ is the identity on the underlying topological space. Hence, an element of $\operatorname{Aut}_{\Sigma}\left(\Sigma^{\prime}\right)$ is a pair of automorphisms $\left(\psi_{y^{\prime}}, \psi_{z^{\prime}}\right) \in \operatorname{Aut}_{C_{y}}\left(C_{y^{\prime}}\right) \times \operatorname{Aut}_{C_{z}}\left(C_{z^{\prime}}\right)$ such that $\psi_{y^{\prime}}, \psi_{z^{\prime}}$ fix the points $0, \infty \in \mathbf{P}_{k}^{1}$. Thus, $\psi_{y^{\prime}}, \psi_{z^{\prime}}= \pm 1$, so $\operatorname{Aut}_{\Sigma}\left(\Sigma^{\prime}\right)=\{ \pm 1\} \times$ $\{ \pm 1\}$. For $x^{\prime}=y^{\prime}, z^{\prime}$, the automorphism $-1: C_{x^{\prime}} \stackrel{\sim}{\longrightarrow} C_{x^{\prime}}$ lifts to the automorphism -1 : $\mathbf{S}(\sqrt{q})_{+} \stackrel{\sim}{\longrightarrow} \mathbf{S}(\sqrt{q})_{+}$. Therefore, the conjugation action of $\operatorname{Aut}_{\Sigma}\left(\Sigma^{\prime}\right)$ on $\mathcal{G}\left(\Sigma^{\prime}, E\right)$ is given as follows:

$$
\begin{array}{r}
(1,1) \cdot( \pm 1, \pm 1)=(-1,-1) \cdot( \pm 1, \pm 1)=( \pm 1, \pm 1) \\
(-1,1) \cdot( \pm 1, \pm 1)=(1,-1) \cdot( \pm 1, \pm 1)=-( \pm 1, \pm 1) .
\end{array}
$$

By Theorem 7.4(2), there are two isomorphism classes of lifts of $\Sigma^{\prime}$ to a tame covering of $(E, V)$, and each such lift has two automorphisms.

These liftings can be described concretely as follows. Fix a square root of $q$, let $E_{ \pm}$be the algebraization of the analytic elliptic curve $\mathbf{G}_{m}^{\text {an }} /( \pm \sqrt{q})^{\mathbf{Z}}$, and let $\psi_{ \pm}: E_{ \pm} \rightarrow E$ be the morphism $t \mapsto t^{2}$ on uniformizations. Let $\Sigma_{ \pm}=\psi_{ \pm}^{-1}(\Sigma)$. Then, $\Sigma_{ \pm}$is isomorphic to $\Sigma^{\prime}$ as a tame covering of $\Sigma$ and $E_{ \pm}$is a lifting of $\Sigma^{\prime}$ to a tame covering of (E,D). The elliptic curves $E_{ \pm}$are not isomorphic (as $K$ schemes) because they have different $q$ invariants, so they represent the two isomorphism classes of liftings of $\Sigma^{\prime}$. The nontrivial automorphism of $E_{ \pm}$is given by translating by the image of $-1 \in \mathbf{G}_{m}^{\text {an }}(K)$ (this is not a homomorphism). In fact, since $\psi_{ \pm}: E_{ \pm} \rightarrow E$ is an étale Galois cover of degree 2, this is the only nontrivial automorphism of $E_{ \pm}$as an $E$-curve; so the homomorphism

$$
\operatorname{Aut}_{E}\left(E_{ \pm}\right) \longrightarrow \operatorname{Aut}_{\Sigma}\left(\Sigma^{\prime}\right) \cong\{ \pm 1\} \times\{ \pm 1\}
$$

is injective but not surjective (its image is $\{ \pm(1,1)\}$ ).

Consider now the automorphism group $\operatorname{Aut}_{\Sigma}^{0}\left(\Sigma^{\prime}\right) \subset \operatorname{Aut}_{\Sigma}\left(\Sigma^{\prime}\right)$ consisting of all degree1 finite harmonic morphisms $\alpha: \Sigma^{\prime} \rightarrow \Sigma^{\prime}$ respecting $\varphi: \Sigma^{\prime} \rightarrow \Sigma$ and inducing the identity on the metric graph $\Gamma^{\prime}$ underlying $\Sigma^{\prime}$. The restriction of the conjugacy action of $\operatorname{Aut}_{\Sigma}\left(\Sigma^{\prime}\right)$ on $\mathcal{G}\left(\Sigma^{\prime}, X\right)$ to the subgroup $\operatorname{Aut}_{\Sigma}^{0}\left(\Sigma^{\prime}\right)$ admits a simplified description that we describe now. Combining this with arguments similar to those in the proof of Theorem 7.4 yields a classification of the set of liftings of $\Sigma^{\prime}$ up to isomorphism as liftings of the metric graph underlying $\Sigma^{\prime}$; see Theorem 7.10.

First, for each $x^{\prime} \in V\left(\Sigma^{\prime}\right)$ with image $x=\varphi\left(x^{\prime}\right)$, let $\operatorname{Aut}_{C_{x}}^{0}\left(C_{x^{\prime}}\right)$ be the subgroup of Aut $_{C_{x}}\left(C_{x^{\prime}}\right)$ that fixes every point of $C_{x^{\prime}}$ of the form red $\left(e^{\prime}\right)$ for some edge $e^{\prime}$ of $\Sigma^{\prime}$ adjacent to $x^{\prime}$. Then,

$$
\operatorname{Aut}_{\Sigma}^{0}\left(\Sigma^{\prime}\right)=\prod_{x^{\prime} \in V_{f}\left(\Sigma^{\prime}\right)} \operatorname{Aut}_{C_{\varphi(x)}}^{0}\left(C_{x^{\prime}}\right)=: \mathcal{E}^{0}
$$

Denote by $\mathcal{E}^{1}$ the finite abelian group

$$
\mathcal{E}^{1}=\prod_{e^{\prime} \in E_{f}\left(\Sigma^{\prime}\right)} \operatorname{Aut}_{\tau^{-1}\left(\varphi\left(e^{\prime}\right)^{\circ}\right)}\left(d_{e^{\prime}}(\varphi)\right) .
$$

The discussion preceding Theorem 7.4 shows that the set of gluing data $\mathcal{G}\left(\Sigma^{\prime}, X\right)$ is canonically a principal homogeneous space under $\mathcal{E}^{1}$. 
The subgroup $\operatorname{Aut}_{C_{x}}^{0}\left(C_{x^{\prime}}\right) \subset \operatorname{Aut}_{C_{x}}\left(C_{x^{\prime}}\right)$ corresponds to the subgroup $\operatorname{Aut}_{Y(x)}^{0}\left(Y^{\prime}\left(x^{\prime}\right)\right)$ of automorphisms in $\operatorname{Aut}_{Y(x)}\left(Y^{\prime}\left(x^{\prime}\right)\right)$ which act trivially on the skeleton $\Sigma^{\prime}\left(x^{\prime}\right)$. Restriction of a $Y(x)$-automorphism of $Y^{\prime}\left(x^{\prime}\right)$ to $\tau_{x^{\prime}}^{-1}\left(e^{\prime \circ}\right)$ defines a homomorphism

$$
\rho_{x^{\prime}, e^{\prime}}: \operatorname{Aut}_{C_{x}}^{0}\left(C_{x^{\prime}}\right)=\operatorname{Aut}_{Y(x)}^{0}\left(Y^{\prime}\left(x^{\prime}\right)\right) \longrightarrow \operatorname{Aut}_{\tau^{-1}\left(e^{\circ}\right)}\left(d_{e^{\prime}}(\varphi)\right) .
$$

Fix an orientation of each finite edge of $\Sigma^{\prime}$. For $x^{\prime} \in V_{f}\left(\Sigma^{\prime}\right)$ with image $x=\varphi\left(x^{\prime}\right)$, let $\rho_{x^{\prime}}:$ Aut $_{C_{x}}^{0}\left(C_{x^{\prime}}\right) \rightarrow \mathcal{E}^{1}$ be the homomorphism whose $e^{\prime}$ coordinate is

$$
\left(\rho_{x^{\prime}}(\alpha)\right)_{e^{\prime}}= \begin{cases}\rho_{x^{\prime}, e^{\prime}}(\alpha) & \text { if } x^{\prime} \text { is the source vertex of } e^{\prime} \\ \rho_{x^{\prime}, e^{\prime}}(\alpha)^{-1} & \text { if } x^{\prime} \text { is the target vertex of } e^{\prime} \\ 1 & \text { if } x^{\prime} \text { is not an endpoint of } e^{\prime}\end{cases}
$$

Taking the product over all $x^{\prime} \in V_{f}\left(\Sigma^{\prime}\right)$ yields a homomorphism $\rho: \mathcal{E}^{0} \rightarrow \mathcal{E}^{1}$. The kernel and cokernel of $\rho$ are independent of the choice of orientations. Viewing $\mathcal{E}^{0} \rightarrow \mathcal{E}^{1}$ as a two-term complex $\mathcal{E}^{\bullet}$ of groups, its cohomology groups are

$$
H^{0}\left(\mathcal{E}^{\bullet}\right)=\operatorname{ker}(\rho) \quad \text { and } \quad H^{1}\left(\mathcal{E}^{\bullet}\right)=\operatorname{coker}(\rho)
$$

Theorem 7.10. Let $(X, V \cup D)$ be a triangulated punctured curve with skeleton $\Sigma=$ $\Sigma(X, V \cup D)$. Assume that $\Sigma$ has no loop edges. Let $\varphi: \Sigma^{\prime} \rightarrow \Sigma$ be a tame covering of metrized compexes of curves.

1. $\mathcal{G}\left(\Sigma^{\prime}, X\right)$ is canonically a principal homogeneous space under $\mathcal{E}^{1}$, and the conjugacy action of $A u t_{\Sigma}^{0}\left(\Sigma^{\prime}\right)$ on $\mathcal{G}\left(\Sigma^{\prime}, X\right)$ is given by the action of the subgroup $\rho\left(\operatorname{Aut}_{\Sigma}^{0}\left(\Sigma^{\prime}\right)\right)=\rho\left(\mathcal{E}^{0}\right) \subseteq \mathcal{E}^{1}$ on $\mathcal{G}\left(\Sigma^{\prime}, X\right)$.

2. The set of liftings of $\Sigma^{\prime}$ up to isomorphism as liftings of the metric graph underlying $\Sigma^{\prime}$ is a principal homogeneous space under $H^{1}\left(\mathcal{E}^{\bullet}\right)$, and the group of automorphisms of a given lifting as a lifting of the metric graph underlying $\Sigma^{\prime}$ is isomorphic to $H^{0}\left(\mathcal{E}^{\bullet}\right)$.

\subsection{Descent to a general ground field}

Let $K_{0}$ be a subfield of $K$, let $X_{0}$ be a smooth, projective, geometrically connected $K_{0}$ curve, and let $D \subset X_{0}\left(K_{0}\right)$ be a finite set. Let $X=X_{0} \otimes_{K_{0}} K$, let $V$ be a strongly semistable vertex set of $(X, D)$, let $\Sigma=\Sigma(X, V \cup D)$, and let $\varphi: \Sigma^{\prime} \rightarrow \Sigma$ be a tame covering of metrized complexes of curves, as in the statement of Theorem 7.4. Let $\varphi: X^{\prime} \rightarrow X$ be a lifting of $\varphi$ to a tame covering of $(X, V \cup D)$. Whereas we take the data of the morphism $\Sigma^{\prime} \rightarrow \Sigma$ to be geometric, i.e. only defined over $K$, the covering $X^{\prime} \rightarrow X$ is in fact defined over a finite, separable extension of $K_{0}$. This follows from the fact that if $U=X \backslash D$ and $U^{\prime}=X^{\prime} \backslash \varphi^{-1}(D)$, then $\varphi: U^{\prime} \rightarrow U$ is a tamely ramified cover of $U$ over $X$ relative to $D$ (see Remarks 2.20(1) and 4.32(1)), along with the following lemma.

Lemma 7.12. Let $K_{0}$ be any field, let $K$ be a separably closed field containing $K_{0}$, let $X_{0}$ be a smooth, projective, geometrically connected $K_{0}$-curve, and let $D \subset X_{0}\left(K_{0}\right)$ be a finite set. Let $X=X_{0} \otimes_{K_{0}} K$, let $\varphi: X^{\prime} \rightarrow X$ be a finite morphism with $X^{\prime}$ smooth and (geometrically) connected, and suppose that $\varphi$ is branched only over $D$, with all ramification degrees prime 
to the characteristic of $K$. Then, there exists a finite, separable extension $K_{1}$ of $K$ and a morphism $\varphi_{1}: X_{1}^{\prime} \rightarrow X_{0} \otimes_{K_{0}} K_{1}$ descending $\varphi$.

Proof. Let $U_{0}=X_{0} \backslash D$, and let $U=X \backslash D$ and $U^{\prime}=X^{\prime} \backslash \varphi^{-1}(D)$. First, suppose that $K_{0}$ is separably closed. By [40], Exposé XIII, Corollaire 2.12, the tamely ramified étale fundamental groups $\pi_{1}^{t}\left(U_{0}\right)$ and $\pi_{1}^{t}(U)$ are isomorphic (with respect to some choice of base point). Since $\varphi: U^{\prime} \rightarrow U$ is a tamely ramified cover of $U$ over $X$ relative to $D$, it is classified by a finite-index subgroup of $\pi_{1}^{t}(U)=\pi_{1}^{t}\left(U_{0}\right)$, so there exists a tamely ramified cover $\varphi_{0}: U_{0}^{\prime} \rightarrow U_{0}$ of $U_{0}$ over $X_{0}$ relative to $D$ descending $\varphi$.

Now, we drop the hypothesis that $K_{0}$ is separably closed. By the previous paragraph, we may assume that $K$ is a separable closure of $K_{0}$. By general principles, the projective morphism $X^{\prime} \rightarrow X$ descends to a subfield of $K$ which is finitely generated (i.e. finite) over $K_{0}$.

Remark 7.13. With the notation in (7.11), suppose that $K_{0}$ is a complete valued field with value group $\Lambda_{0}=\operatorname{val}\left(K_{0}^{\times}\right)$and algebraically closed residue field $k$; that $\Sigma$ is 'rational over $K_{0}$ ' in that it comes from a (split) semistable formal $R_{0}$-model of $X_{0}$ in the sense of Section 5, and that $\Sigma^{\prime}$ has edge lengths contained in $\Lambda_{0}$. With some extra work, it is possible to carry out the gluing arguments of Theorem 7.7 directly over the field $K_{0}$ (in this context Lemma 6.10(1) still holds), which shows that the cover $X^{\prime} \rightarrow X$ is in fact defined over $K_{0}$. In the case of a discrete valuation, this also follows from [47] or from [39] if $D=\emptyset$.

\subsection{Liftings of tame harmonic morphisms}

Theorem 7.7 implies the existing of liftings for (finite) tame harmonic morphisms of metrized complexes which are not necessarily tame coverings, the difference being generic étaleness. See Definition 2.21 for both definitions.

Proposition 7.15. Let $(X, V \cup D)$ be a triangulated punctured $K$-curve, let $\Sigma=\Sigma(X, V \cup$ $D)$, and let $\varphi: \Sigma^{\prime} \rightarrow \Sigma$ be a tame harmonic morphism of metrized complexes of curves. Then, there exists a triangulated punctured $K$-curve $\left(X^{\prime}, V^{\prime} \cup D^{\prime}\right)$ and a finite morphism $\psi:\left(X^{\prime}, V^{\prime} \cup D^{\prime}\right) \rightarrow(X, V \cup D)$ lifting $\varphi$.

Proof. For any finite vertex $x^{\prime}$ of $\Sigma^{\prime}$ at which $\varphi$, seen as a morphism of augmented metric graphs, is ramified, let $q_{1}, \ldots, q_{r} \in C_{\nu}$ be all the branch points of $\varphi_{x^{\prime}}$ which do not correspond to any edge of $\Sigma$, and let $p_{i j}$ denote the preimages of $q_{i}$ under $\varphi_{x^{\prime}}$. We modify $\Sigma^{\prime}$ and $\Sigma$ by attaching infinite edges $e_{i}$ to $\Sigma$ at $x=\varphi\left(x^{\prime}\right)$ for each $q_{i}$, infinite edges $e_{i j}^{\prime}$ to $\Sigma^{\prime}$ at $x^{\prime}$ for each $p_{i j}$, and defining $\operatorname{red}_{x}\left(e_{i}\right)=q_{i}$ and $\operatorname{red}_{x^{\prime}}\left(e_{i j}^{\prime}\right)=p_{i j}$. Since each $\varphi_{x^{\prime}}$ is tamely ramified, the harmonic morphism $\varphi$ naturally extends to a tame covering $\widetilde{\varphi}: \widetilde{\Sigma}^{\prime} \rightarrow \widetilde{\Sigma}$ between the resulting modifications. Enlarging $D$ to $\widetilde{D}$ by choosing points in $X(K) \backslash D$ with reduction $q_{i}$, we can assume that $\widetilde{\Sigma}=\Sigma(X, V \cup \widetilde{D})$. The result now follows from Theorem 7.7 by first lifting $\widetilde{\varphi}$ to a tame covering $\psi:\left(X^{\prime}, V^{\prime} \cup \widetilde{D}^{\prime}\right) \rightarrow(X, V \cup \widetilde{D})$ and then taking the restriction of $\psi$ to $\left(X^{\prime}, V^{\prime} \cup D^{\prime}\right) \rightarrow(X, V \cup D)$, where $D^{\prime}=\psi^{-1}(D)$.

Remark 7.16. As mentioned above, in Proposition 7.15 we do not require that $\varphi: \Sigma^{\prime} \rightarrow$ $\Sigma$ be generically étale. This corresponds to not requiring that $D \subset X(K)$ contain the 
branch locus of the lift $\psi: X^{\prime} \rightarrow X$ : indeed, by Lemma 4.33, if $D$ contains the branch locus then $\varphi$ is a tame covering. In the situation of Proposition 7.15, the set of liftings of $\Sigma^{\prime}$ to a tame covering of $(X, V \cup D)$ can be infinite. For example, if $\Gamma^{\prime}=\left\{p^{\prime}\right\}$ and $\Gamma=\{p\}$ are both points and the morphism $\varphi_{p^{\prime}}: C_{p^{\prime}} \cong \mathbf{P}^{1} \rightarrow C_{p} \cong \mathbf{P}^{1}$ is $z \mapsto z^{2}$, with $(X, V)$ a minimal triangulation of $\mathbf{P}^{1}$ and $D=\emptyset$, then there are infinitely many such lifts, corresponding to the different ways of lifting the critical points and critical values of $\varphi_{p^{\prime}}$ from $k$ to $K$.

\section{Application: component groups of Néron models}

In contrast to the rest of the paper, we assume in this section that $R$ is a complete discrete valuation ring with fraction field $K$ and algebraically closed residue field $k$. In this case, we take the value group $\Lambda$ to be $\mathbf{Z}$.

There is a natural notion of harmonic 1 forms on a metric graph $\Gamma$ of genus $g$ (see [37]). The space $\Omega^{1}(\Gamma)$ is a $g$-dimensional real vector space which can be canonically identified with $H^{1}(\Gamma, \mathbf{R})$, but we write elements of $\Omega^{1}(\Gamma)$ as $\omega=\sum_{e} \omega_{e} d e$ as in [7], where the sum is over all edges with respect to a fixed vertex set for $\Gamma$. There is a canonical lattice $\Omega_{\mathbf{Z}}^{1}(\Gamma)$ of integer harmonic 1-forms inside $\Omega^{1}(\Gamma)$; these are the harmonic 1-forms for which every $\omega_{e}$ is an integer. A harmonic morphism $\varphi: \Gamma^{\prime} \rightarrow \Gamma$ induces a natural pullback map on harmonic 1-forms via the formula

$$
\varphi^{*}\left(\sum_{e} \omega_{e} d e\right)=\sum_{e^{\prime}} d_{e^{\prime}}(\varphi) \cdot \omega_{\varphi\left(e^{\prime}\right)} d e^{\prime} .
$$

Recall that a $\mathbf{Z}$-metric graph (i.e. a $\Lambda$-metric graph for $\Lambda=\mathbf{Z}$ ) with no infinite vertices is a (compact and finite) metric graph whose edge lengths are all positive integers (or equivalently, having a vertex set with respect to which all edge lengths are 1 ). If $X / K$ is a smooth, proper, geometrically connected analytic curve and $\mathfrak{X}$ is a semistable $R$-model for $X$, the skeleton $\Gamma_{\mathfrak{X}}$ of $\mathfrak{X}$ is naturally a $\mathbf{Z}$-metric graph. Moreover, as we have seen earlier in this paper, a finite morphism of semistable models induces in a natural way a harmonic morphism of $\mathbf{Z}$-metric graphs.

Let $\Gamma$ be a $\mathbf{Z}$-metric graph with no infinite vertices. We define the regularized Jacobian $\operatorname{Jac}_{\text {reg }}(\Gamma)$ of $\Gamma$ to be the group $\operatorname{Jac}(G)$, where $G$ is the 'regular model' for $\Gamma$ (the unique graph induced by a vertex set with all edge lengths equal to 1$)$. The group $\operatorname{Jac}(G)$ can be described explicitly as

$$
\operatorname{Jac}(G)=\Omega^{1}(G)^{\#} / H_{1}(G, \mathbf{Z}),
$$

where $\Omega^{1}(G)^{\#}$ denotes the linear functionals $\Omega^{1}(G, \mathbf{R}) \rightarrow \mathbf{R}$ of the form $\int_{\alpha}$ with $\alpha \in$ $C_{1}(G, \mathbf{Z})$ (see [7]). There is a canonical isomorphism between $\operatorname{Jac}(G)$ and $\operatorname{Pic}^{0}(G)$, the group of divisors of degree 0 on $G$ modulo the principal divisors (see [5]) as well as a canonical isomorphism

$$
\operatorname{Jac}(G) \cong H^{1}(G, \mathbf{Z})^{\#} / \Omega_{\mathbf{Z}}^{1}(G),
$$

where

$$
H^{1}(G, \mathbf{Z})^{\#}=\left\{\omega \in \Omega^{1}(G, \mathbf{R}): \int_{\gamma} \omega \in \mathbf{Z} \forall \gamma \in H_{1}(G, \mathbf{Z})\right\} .
$$

We recall (in our own terminology) the following result of Raynaud (cf. [38] and ([6], Appendix A)): 
Theorem 8.2 (Raynaud). If $X / K$ is a semistable curve, then the component group of the Néron model of Jac $(X)$ over $R$ is canonically isomorphic to Jac reg $\left(\Gamma_{\mathfrak{X}}\right)$ (for any semistable model $\mathfrak{X}$ of $X$ ).

A harmonic morphism $\varphi: \Gamma^{\prime} \rightarrow \Gamma$ of $\mathbf{Z}$-metric graphs induces in a functorial way homomorphisms $\varphi_{*}: \operatorname{Jac}_{\mathrm{reg}}\left(\Gamma^{\prime}\right) \rightarrow \operatorname{Jac}_{\mathrm{reg}}(\Gamma)$, defined by

$$
\varphi_{*}\left(\left[\int_{\alpha^{\prime}}\right]\right)=\left[\int_{\varphi_{*}\left(\alpha^{\prime}\right)}\right],
$$

where

$$
\varphi_{*}\left(\sum \alpha_{e^{\prime} e^{\prime}}\right)=\sum \alpha_{e^{\prime}} \varphi\left(e^{\prime}\right),
$$

and $\varphi^{*}: \operatorname{Jac}_{\mathrm{reg}}(\Gamma) \rightarrow \operatorname{Jac}_{\mathrm{reg}}\left(\Gamma^{\prime}\right)$, defined by

$$
\varphi^{*}([\omega])=\left[\varphi^{*} \omega\right]
$$

where

$$
\varphi^{*}\left(\sum \omega_{e} d e\right)=\sum_{e^{\prime}} d_{e^{\prime}}(\varphi) \omega_{\varphi\left(e^{\prime}\right)} d e^{\prime} .
$$

We have the following elementary result, whose proof we omit:

Lemma 8.3. Under the canonical isomorphism between $\operatorname{Jac}(G)$ and $\operatorname{Pic}^{0}(G)$, the homomorphism $\varphi_{*}: \operatorname{Jac}\left(G^{\prime}\right) \rightarrow \operatorname{Jac}(G)$ corresponds to the map $\left[D^{\prime}\right] \mapsto\left[\varphi_{*}\left(D^{\prime}\right)\right]$ from Pic ${ }^{0}\left(G^{\prime}\right)$ to $\operatorname{Pic}^{0}(G)$, where $\varphi_{*}\left(\sum a_{v^{\prime}}\left(v^{\prime}\right)\right)=\sum a_{v^{\prime}} \varphi\left(v^{\prime}\right)$.

The following is a 'relative' version of Raynaud's theorem; it implies that the covariant functor which takes a semistable $R$-model $\mathfrak{X}$ for a $K$-curve $X$ to the component group of the Néron model of the Jacobian of $X$ factors as the 'reduction graph functor' $\mathfrak{X} \mapsto$ $\Gamma_{\mathfrak{X}}$ followed by the 'regularized Jacobian functor' $\Gamma \mapsto \operatorname{Jac}_{\mathrm{reg}}(\Gamma)$. It is a straightforward consequence of the analytic description of Raynaud's theorem given in [6], Appendix A (see also [13]):

Theorem 8.4. If $f_{K}: X^{\prime} \rightarrow X$ is a finite morphism of curves over $K$, the induced maps $f_{*}: \Phi_{J\left(X^{\prime}\right)} \rightarrow \Phi_{J(X)}$ and $f^{*}: \Phi_{J(X)} \rightarrow \Phi_{J\left(X^{\prime}\right)}$ on component groups coincide with the induced maps $\varphi_{*}: \operatorname{Jac}_{\text {reg }}\left(\Gamma_{\mathfrak{X}^{\prime}}\right) \rightarrow \operatorname{Jac}_{\text {reg }}\left(\Gamma_{\mathfrak{X}}\right)$ and $\varphi^{*}: \operatorname{Jac}_{\text {reg }}\left(\Gamma_{\mathfrak{X}}\right) \rightarrow \operatorname{Jac}_{\text {reg }}\left(\Gamma_{\mathfrak{X}^{\prime}}\right)$ for any morphism $f: \mathfrak{X}^{\prime} \rightarrow \mathfrak{X}$ of semistable models extending $f_{K}$. (Here, $\varphi$ denotes the harmonic morphism of skeleta induced by f; see Remark 5.14.)

It follows easily from Lemma 8.3 and Theorem 8.4 that if $f_{K}: X^{\prime} \rightarrow X$ is regularizable, i.e., if $f_{K}$ extends to a morphism of regular semistable models, then the induced map $f_{*}$ : $\Phi_{X^{\prime}} \rightarrow \Phi_{X}$ on component groups is surjective. Thus whenever $f_{*}$ is not surjective, it follows that $f_{K}$ does not extend to a morphism of regular semistable models. One can obtain a number of concrete examples of this situation from modular curves (e.g. the map $X_{0}(33) \rightarrow E$ over $\mathbf{Q}_{3}^{\text {unr }}$, where $E$ is the optimal elliptic curve of level 33.)

Remark 8.5. One can show that if $\varphi: \Gamma^{\prime} \rightarrow \Gamma$ is a harmonic morphism of Z-metric graphs, then $\varphi_{*}: \Phi_{X^{\prime}} \rightarrow \Phi_{X}$ is surjective iff $\varphi^{*}: \Phi_{X} \rightarrow \Phi_{X^{\prime}}$ is injective. Indeed, 
it is not hard to check that the maps $\varphi_{*}$ and $\varphi^{*}$ are adjoint with respect to the combinatorial monodromy pairing, the non-degenerate symmetric bilinear form $\langle$,$\rangle :$ $\operatorname{Jac}(G) \times \operatorname{Jac}(G) \rightarrow \mathbf{Q} / \mathbf{Z}$ defined by $\left\langle[\omega],\left[\int_{\alpha}\right]\right\rangle=\left[\int_{\alpha} \omega\right]$, where $\omega \in H_{1}(G, \mathbf{Z})^{\#}$ and $\int_{\alpha} \in$ $\Omega^{1}(G)^{\#}$. Thus, the groups $\operatorname{ker}\left(\varphi^{*}\right)$ and $\operatorname{coker}\left(\varphi_{*}\right)$ are canonically dual. This is a combinatorial analogue of results proved by Grothendieck in SGA7 on the (usual) monodromy pairing.

As an application of Theorem 8.4 and our results on lifting harmonic morphisms, one can construct many examples of harmonic morphisms of $\mathbf{Z}$-metric graphs for which $\varphi_{*}$ is not surjective. For example, consider the following question posed by Ken Ribet in a 2007 email correspondence with the second author (Baker):

Suppose $f: X^{\prime} \rightarrow X$ is a finite morphism of semistable curves over a complete discretely valued field $K$ with $g(X) \geq 2$. Assume that the special fiber of the minimal regular model of $X^{\prime}$ consists of two projective lines intersecting transversely. Is the induced map $f_{*}: \Phi_{X^{\prime}} \rightarrow \Phi_{X}$ on component groups of Néron models necessarily surjective?

We now show that the answer to Ribet's question is no.

Example 8.6. Consider the 'banana graph' $B\left(\ell_{1}, \ldots, \ell_{g+1}\right)$ consisting of two vertices and $g+1$ edges of length $\ell_{i}$. This is the reduction graph of a semistable curve whose reduction has two $\mathbf{P}^{1}$ 's crossing transversely at singular points of thickness $\ell_{1}, \ldots, \ell_{g+1}$. If we set $G^{\prime}=B(1,1,1,1)$ and $G=B(1,2,2)$ and let $\Gamma^{\prime}$, $\Gamma$ be the geometric realizations of $G^{\prime}$ and $G$, respectively, then there is a degree 2 harmonic morphism of $\mathbf{Z}$-metric graphs $\varphi: \Gamma^{\prime} \rightarrow \Gamma$ taking $e_{1}^{\prime}$ and $e_{2}^{\prime}$ to $e_{1}, e_{3}^{\prime}$ to $e_{2}$, and $e_{4}^{\prime}$ to $e_{3}$. The homomorphism $\varphi_{*}$ is nonsurjective since $\left|\mathrm{Jac}_{\mathrm{reg}}(\Gamma)\right|=4$ and $\left|\mathrm{Jac}_{\mathrm{reg}}\left(\Gamma^{\prime}\right)\right|=8$. The map $\varphi$ can be enriched to a harmonic morphism $\tilde{\varphi}$ of metrized complexes of curves by attaching a $\mathbf{P}^{1}$ to each vertex and letting each morphism $\mathbf{P}^{1} \rightarrow \mathbf{P}^{1}$ be $z \mapsto z^{2}$, with $0, \pm 1$, and $\infty$ being the marked points upstairs. By Remark 7.13, the morphism $\tilde{\varphi}$ lifts to a morphism $\psi: X^{\prime} \rightarrow X$ of curves over $K$. Since all edges of $G^{\prime}$ have length 1, the minimal proper regular model of $X^{\prime}$ has a special fiber consisting of two $\mathbf{P}^{1}$ 's crossing transversely. By Theorem 8.4, the induced covariant map on component groups of Néron models is not surjective.

\section{Endnotes}

${ }^{a}$ This isomorphism is not canonical. However, there is an isomorphism $\operatorname{Aut}_{A}\left(A^{\prime}\right) \cong \boldsymbol{\mu}_{\delta}$ which is canonical up to the action of $\mathbf{Z} / 2 \mathbf{Z}$ on $\operatorname{Aut}_{A}\left(A^{\prime}\right)$ sending $\sigma$ to $\sigma^{-1}$. Equivalently, the parameters $t$ and $t^{-1}$ give rise to different homomorphisms $\sigma \mapsto \sigma\left(t^{ \pm 1}\right) / t^{ \pm 1}: \operatorname{Aut}_{A}\left(A^{\prime}\right) \rightarrow \boldsymbol{\mu}_{\delta}$, but the choice of an orientation for (the skeleton of) $A$ induces a canonical isomorphism $\operatorname{Aut}_{A}\left(A^{\prime}\right) \cong \boldsymbol{\mu}_{\delta}$.

${ }^{\mathrm{b}}$ This $\delta$ need not be prime to $\operatorname{char}(k)$.

${ }^{\mathrm{c}}$ We thank the referee for greatly simplifying our original proof of Lemma 6.19.

\section{Acknowledgments}

The authors thank Amaury Thuillier and Antoine Ducros for their help with some of the descent arguments in (5.2). We are grateful to Andrew Obus for a number of useful comments based on a careful reading of the first arXiv version of this manuscript. We are also very grateful to the referee for quickly and thoroughly checking all of the technical arguments in the paper, pointing out several errors and suggesting many improvements. MB was partially supported by NSF grant DMS-1201473. EB was partially supported by the ANR-09-BLAN-0039-01. 


\section{Author details}

${ }^{1}$ CNRS - Département de mathématiques et applications, École Normale Supérieure, 45 Rue d'Ulm, Paris. ${ }^{2}$ School of Mathematics, Georgia Institute of Technology, Atlanta GA 30332-0160, USA. ${ }^{3}$ Université Pierre et Marie Curie, Paris 6, 4 Place Jussieu, 75005 Paris, France.

Received: 9 December 2014 Accepted: 30 December 2014

Published online: 10 June 2015

\section{References}

1. Abramovich, S, Caporaso, L, Payne, S: The tropicalization of the moduli space of curves. to appear in Ann. Sci. Éc. Norm. Supér., preprint available at http://arxiv.org/abs/1212.0373

2. Amini, $\mathrm{O}$, Baker, $\mathrm{M}$ : Linear series on metrized complexes of algebraic curves. to appear in Math. Ann., preprint available at http://arxiv.org/abs/1204.3508

3. Amini, O, Baker, M, Brugallé, E, Rabinoff, J: Lifting harmonic morphisms II: tropical curves and metrized complexes. Algebra and Number Theory. 9-2, 267-315 (2015). doi:10.2140/ant.2015.9.267

4. Anand CK: A discrete analogue of the harmonic morphism, Harmonic morphisms, harmonic maps, and related topics (Brest, 1997), Chapman \& Hall/CRC Res. Notes Math. 413, 109-112 (2000)

5. Bacher, R, de la Harpe, P, Nagnibeda, T: The lattice of integral flows and the lattice of integral cuts on a finite graph Bull. Soc. Math. France. 125(2), 167-198 (1997)

6. Baker, M: Specialization of linear systems from curves to graphs. Algebra Number Theory. 2(6), 613-653 (2008). With an appendix by Brian Conrad

7. Baker, M, Faber, X: Metric properties of the tropical Abel-Jacobi map. J. Algebraic Combin. 33(3), 349-381 (2011)

8. Baker, M, Norine, S: Harmonic morphisms and hyperelliptic graphs. Int. Math. Res. Notices. 15, 2914-2955 (2009)

9. Baker, M, Rumely, R: Potential theory and dynamics on the Berkovich projective line. Mathematical Surveys and Monographs, vol. 159, American Mathematical Society, Providence, RI, 2010

10. Baker, M, Norine, S: Riemann-Roch and Abel-Jacobi theory on a finite graph. Adv. Math. 215(1), 766-788 (2007)

11. Baker, M, Payne, S, Rabinoff, J: Nonarchimedean geometry, tropicalization, and metrics on curves (2011). Preprint available at http://arxiv.org/abs/1104.0320v2

12. Baker, M, Payne, S, Rabinoff, J: On the structure of non-Archimedean analytic curves. Tropical and non-A, rchimedean geometry. Contemp. Math., vol. 605, Amer. Math. Soc., Providence, Rl, 2013, p. 93-121, 3204269

13. Baker, M, Rabinoff, J: The skeleton of the Jacobian, the Jacobian of the skeleton, and lifting meromorphic functions from tropical to algebraic curves. to appear in International Math. Research Notices., preprint available at http://arxiv. org/abs/1308.3864

14. Berkovich, VG: Spectral theory and analytic geometry over non-Archimedean fields. Mathematical Surveys and Monographs, vol 33, American Mathematical Society, Providence, RI, 1990

15. Berkovich, VG: Étale cohomology for non-Archimedean analytic spaces. Inst. Hautes É, tudes Sci. Publ. Math. 78, 5-161 (1993)

16. Berkovich, VG: Smooth $p$-adic analytic spaces are locally contractible. Invent. Math. 137(1), 1-84 (1999)

17. Bertrand, B, Brugallé, E, Mikhalkin, G: Tropical open Hurwitz numbers. Rend. Semin. Mat. Univ. Padova. 125, 157-171 (2011)

18. Bosch, S, Lütkebohmert, W: Stable reduction and uniformization of abelian varieties. I. Math. Ann. 270(3), 349-379 (1985)

19. Bosch, S, Lütkebohmert, W: Formal and rigid geometry. I. Rigid spaces. Math. Ann. 295(2), 291-317 (1993)

20. Bosch, S, Güntzer, U, Remmert, R: Non-Archimedean analysis. Grundlehren der Mathematischen Wissenschaften [Fundamental Principles of Mathematical Sciences], Vol. 261. Springer-Verlag, Berlin (1984)

21. Chan, M: Tropical hyperelliptic curves. J. Alg. Combin. 15, 2914-2955 (2012)

22. Conrad, B: Irreducible components of rigid spaces. Ann. Inst. Fourier (Grenoble). 49(2), 473-541 (1999)

23. Coleman, RF: Stable maps of curves. Documenta Math. Extra volume Kato, 217-225 (2003)

24. Cornelissen, G, Kato, F, Kool, J: A combinatorial Li-Yau inequality and rational points on curves. to appear in Math. Ann., preprint available at http://arxiv.org/abs/1211.2681

25. Ducros, A: La structure des courbes analytiques. Preliminary version available at http://webusers.imj-prg.fr/ antoine. ducros/livre.html

26. Ducros, A: Triangulations et cohomologie étale sur une courbe analytique $p$-adique. J. Algebraic Geom. 17(3), 503-575 (2008)

27. Faber, X: Topology and geometry of the Berkovich ramification locus for rational functions. Manuscripta Math. 142(3-4), 439-474 (2013)

28. Faber, X: Topology and geometry of the Berkovich ramification locus for rational functions, II. Math. Ann. 356(3), 819-844 (2013)

29. Harris, J, Mumford, D: On the Kodaira dimension of the moduli space of curves. Invent. Math. 67(1), 23-88 (1982) With an appendix by W. Fulton

30. Kontsevich, M, Soibelman, Y: Homological mirror symmetry and torus fibrations. Symplectic geometry and mirror symmetry (Seoul, 2000). World Sci, Publishing, River Edge, NJ (2001)

31. Kontsevich, M, Soibelman, Y: Affine structures and non-Archimedean analytic spaces. The unity of mathematics, Progr. Math., Vol. 244, Birkhäuser Boston, Boston, MA (321)

32. Liu, Q, Lorenzini, D: Models of curves and finite covers. Compositio Math. 118(1), 61-102 (1999)

33. Liu, Q: Reduction and lifting of finite covers of curves. In: Proceedings of the 2003. Workshop on Cryptography and Related Mathematics, Chuo University, pp. 161-180, (2003)

34. Liu, Q: Stable reduction of finite covers of curves. Compositio Math. 142(1), 101-118 (2006)

35. Mikhalkin, G: Tropical geometry and its applications. In: International Congress of Mathematicians. Vol. II, Eur. Math. Soc, pp. 827-852, Zürich, (2006)

36. Mikhalkin, G: Enumerative tropical algebraic geometry in $\mathbb{R}^{2}$. J. Amer. Math. Soc. 18(2), 313-377 (2005) 
37. Mikhalkin, G, Zharkov, I: Tropical curves, their Jacobians and theta functions. Curves and abelian varieties, Contemp. Math, Vol. 465, Amer. Math. Soc., Providence, RI (2008)

38. Raynaud, M: Spécialisation du foncteur de Picard. Inst. Hautes Études Sci. Publ. Math. 38, $27-76$ (1970)

39. Saïdi, M: Revêtements modérés et groupe fondamental de graphe de groupes. Compos. Math. 107, 319-338 (1997)

40. Grothendieck, A, Raynaud, M: Revêtements étales et groupe fondamental (SGA 1), Séminaire de Géométrie Algébrique de l'Institut des Hautes Études Scientifiques (SGA 1), Société Mathématique de France, Paris (2003)

41. Saïdi, M: Revêtements étales abéliens, courants sur les graphes et réductions semi-stable des courbes. Manuscripta Math. 89, 245-265 (1996)

42. Saïdi, M: Wild ramification and a vanishing cycles formula. J. Algebra. 273(1), 108-128 (2004)

43. Temkin, M: Introduction to Berkovich analytic spaces. Preprint available at http://arxiv.org/abs/1010.2235v1

44. Thuillier, A: Théorie du potentiel sur les courbes en géométrie analytique non archimédienne. Applications à la théorie d'Arakelov. Ph.D. thesis, University of Rennes (2005). Preprint available at http://tel.archives-ouvertes.fr/docs/ 00/04/87/50/PDF/tel-00010990.pdf

45. Urakawa, $\mathrm{H}$ : A discrete analogue of the harmonic morphism and green kernel comparison theorems. Glasg. Math. J. 42, 319-334 (2000)

46. Welliaveetil, J: A Riemann-Hurwitz formula for skeleta in non-Archimedean geometry (2013). Preprint available at http://arxiv.org/abs/1303.0164

47. Wewers, S: Deformation of tame admissible covers of curves. Aspects of Galois theory (Gainesville, FL, 1996), London Math. Soc. Lecture Note Ser, Vol. 256. Cambridge Univ. Press, Cambridge (1999)

\section{Submit your manuscript to a SpringerOpen ${ }^{\circ}$ journal and benefit from:}

- Convenient online submission

- Rigorous peer review

- Immediate publication on acceptance

- Open access: articles freely available online

- High visibility within the field

Retaining the copyright to your article

Submit your next manuscript at $\gg$ springeropen.com 